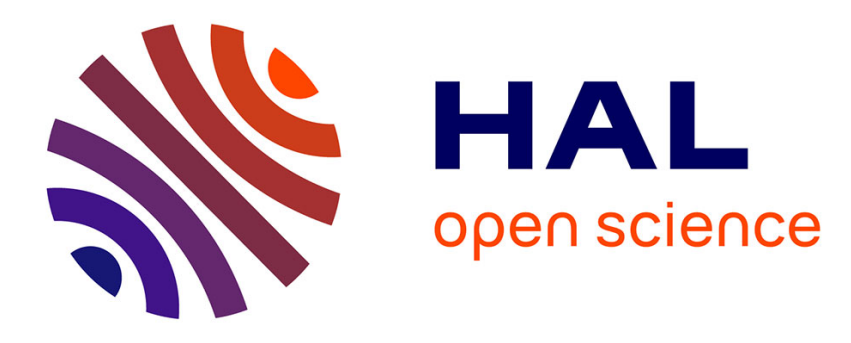

\title{
Recent progress in the development of the Elliptic Blending Reynolds-stress model
}

\author{
Remi Manceau
}

\section{To cite this version:}

Remi Manceau. Recent progress in the development of the Elliptic Blending Reynolds-stress model. International Journal of Heat and Fluid Flow, 2015, pp.32. 10.1016/j.ijheatfluidflow.2014.09.002 . hal-01092931

\section{HAL Id: hal-01092931 \\ https://hal.inria.fr/hal-01092931}

Submitted on 9 Dec 2014

HAL is a multi-disciplinary open access archive for the deposit and dissemination of scientific research documents, whether they are published or not. The documents may come from teaching and research institutions in France or abroad, or from public or private research centers.
L'archive ouverte pluridisciplinaire HAL, est destinée au dépôt et à la diffusion de documents scientifiques de niveau recherche, publiés ou non, émanant des établissements d'enseignement et de recherche français ou étrangers, des laboratoires publics ou privés. 


\title{
Recent progress in the development of the Elliptic Blending Reynolds-stress model
}

\author{
Rémi Manceau \\ Department of Applied Mathematics, CNRS-University of Pau-Inria, avenue de l'université, 64013 Pau, France
}

\begin{abstract}
The Elliptic Blending Reynolds Stress Model (EB-RSM), originally proposed by Manceau and Hanjalić (2002) to extend standard, weakly inhomogeneous Reynolds stress models to the near-wall region, has been subject to various modifications by several authors during the last decade, mainly for numerical robustness reasons. The present work revisits all these modifications from the theoretical standpoint and investigates in detail their influence on the reproduction of the physical mechanisms at the origin of the influence of the wall on turbulence. The analysis exploits recent DNS databases for high-Reynolds number channel flows, spanwise rotating channel flows with strong rotation rates, up to complete laminarization, and the separated flow after a sudden expansion without and with system rotation. Theoretical arguments and comparison with DNS results lead to the selection of a recommended formulation for the EB-RSM model. This formulation shows satisfactory predictions for the configurations described above, in particular as regards the modification of the mean flow and turbulent anisotropy on the anticyclonic or pressure side.
\end{abstract}

\section{Introduction}

Since the pioneering work of Chou (1945), second-moment closures of the RANS (Reynolds-Averaged Navier-Stokes) equations have been essentially founded upon a theoretical framework valid in homogeneous or weakly inhomogeneous turbulence. However, in the vicinity of solid boundaries, turbulence exhibits specific properties that are drastically different from those observed in homogeneous flows. In turbulence modelling, some of these features cannot be overlooked: scales separation is not sufficient to neglect the influence of molecular viscosity on the large, energetic, scales and of large-scale anisotropy on the small, dissipative, scales; the blockage of the wall-normal fluctuations is at the origin of a strong departure from isotropy, and leads to a two-component state of turbulence at the wall; through pressure fluctuations, turbulence loses its local character and feels the presence of the wall in a region much larger than the viscosity affected region.

These features are not accounted for by standard models based on weakly inhomogeneous assumptions, which are consequently not valid in the near-wall region and require the use of wall functions. Various approaches have been proposed in the literature to extend the validity of these models to near-wall regions (for a review of the state of the art, see Hanjalić and Launder, 2011). One of the most successful methods, proposed by Durbin (1991), consists in solving an Elliptic Relaxation equation for the velocity-pressure gradient correlation tensor involved in

Email address: remi.manceau@univ-pau.fr (Rémi Manceau) the Reynolds-stress transport equation, in order to account for the crucial wall blockage. This method is based on solid theoretical foundations and is supported by DNS data (Manceau et al., 2001), but introduces 13 additional differential equations (for the Reynolds stresses, the dissipation rate and the six components of the velocity-pressure gradient correlation) and is numerically stiff because of the boundary conditions of the Elliptic Relaxation equations. In order to reduce the number of equations and to circumvent numerical difficulties, Manceau and Hanjalić (2002) proposed the Elliptic Blending approach, in which the six Elliptic Relaxation equations are replaced by a single equation for a wall-sensitive non-dimensional scalar $\alpha$, with the simple boundary conditions $\alpha=0$ at the wall.

However, the first applications of the Elliptic Blending strategy to more complex configurations (Shin et al., 2003; Thielen et al., 2005; Manceau, 2005) highlighted some deficiencies of the model, which is at the origin of various modifications of the original model that are summarized in section 4 . These modifications mainly affect the formulations of the model for the velocity-pressure gradient tensor, the blending function used to migrate from the near-wall form to the weakly inhomogeneous form of the model and the dissipation equation. On the one hand, such a variability is evidence that the model is alive and applied to practical configurations, but, on the other hand, it is a source of confusion, since the appellation Elliptic Blending Reynolds Stress Model (EB-RSM) actually refers to numerous, slightly different models. My personal experience has shown that new users are often confused by this variability and request information about the version they 
must start with.

Consequently, the main objective of the present article, after more than a decade of developments and applications of the approach by several authors, is to revisit all these modifications, from their theoretical justification to their practical application, in order to investigate their relevance in terms of representation of the near-wall physics. The present work is thus targeted at formulating a reference EB-RSM, with a proper set of coefficients. Since validating this model against the wide range of applications already treated with EB-RSM in the past would be a formidable task, the present paper is focused on the detailed investigation of the reproduction of the main physical mechanisms in a few canonical channel and separated flows, including cases with spanwise rotation, which are relevant to many applications, from turbomachinery to geophysical flows, using, among others, very recent DNS databases that have not been exploited yet in the context of RANS modelling (Lozano-Durán and Jiménez, 2014; Lamballais, 2014).

\section{Physical background}

As a preliminary to the introduction of turbulence models dedicated to the reproduction of the near-wall region, it is useful to summarize the various effects exerted by solid boundaries on the turbulent field and how they can be accounted for in Reynolds stress models. In particular, the distinction between viscous and non-viscous effects is favourable to the interpretation of the role played by the Elliptic Relaxation and Elliptic Blending approaches.

(i) Due to the no-slip boundary condition linked to the viscous character of the fluid, and the associated strong velocity gradients, a peak of turbulence production occurs in the near-wall region. In the framework of near-wall models, this effect is easily reproduced by accounting for the exact boundary condition for the mean velocity $U_{i}=0$. However, it should be pointed out that the quasi-homogeneous hypothesis (Chou, 1945), i.e., the local approximation of the mean velocity gradient by a constant in the rapid part of the redistribution term, is consequently not valid in the near-wall region (Bradshaw et al., 1987). It will be seen below that this approximation is not used in the near-wall region in the Elliptic Relaxation and Elliptic Blending models.

(ii) The no-slip condition applied to the velocity fluctuations leads to a viscous damping of the Reynolds stresses, which makes necessary the accounting of viscous terms in the Reynolds-stress transport equations: the Reynolds stresses behave as $y^{2}$, if $y$ represents the direction normal to a wall located in $y=0$ (as shown below, the behaviour in $y^{n}$, with $n>2$, of some components is due to the blockage effect). The boundary condition $\overline{u_{i} u_{j}}=0$ is not sufficient to impose this behaviour, which requires the correct reproduction of the balance between viscous diffusion and dissipation in the Reynolds stress transport equations in the vicinity of the wall. This requirement is consequently linked to the correct modelling of the dissipation tensor: since the viscous damping also suppresses the scale separation between energetic and dissipative structures, the anisotropy of the dissipation tensor cannot be neglected. In practice, the correct asymptotic behaviour is obtained in nearwall Reynolds-stress models by choosing a model for the dissipation tensor that satisfies

$\lim _{y \rightarrow 0} \varepsilon_{i j}=2 \nu \lim _{y \rightarrow 0} \frac{\overline{u_{i} u_{j}}}{y^{2}}$.

(iii) In the case of an incompressible flow, the continuity equation implies that the fluctuating velocity normal to an impermeable surface, $v$, behaves as $y^{n+1}$, where $n$ is the exponent describing the asymptotic behaviour $\left(y^{n}\right)$ of the tangential components $u$ and $w$ (for details, see appendix Appendix A). As a consequence, the Reynolds stress component $\overline{v^{2}}$ behaves as $y^{2 n+2}$, and is asymptotically negligible compared to the other two normal stresses, $\overline{u^{2}}$ and $\overline{w^{2}}$, which behave as $y^{2 n}$. This effect is purely kinematic, independent of the viscous character of the fluid, and is observed for solid walls as well as free-slip surfaces (Yokojima and Shima, 2010). Therefore, for models that are able to reproduce the correct behaviour of turbulence in the presence of solid walls, the ambiguous designation low-Reynolds number models should be avoided and replaced by near-wall models. For a free-slip surface, components $\overline{u^{2}}$ and $\overline{w^{2}}$ behave as $y^{0}$ and $\overline{v^{2}}$ as $y^{2}$; for a no-slip surface, this asymptotic behaviour is changed to $y^{2}$ and $y^{4}$, respectively. In all cases, the most important effect to take into account is the fact that $\overline{v^{2}}$ is negligible compared to the other normal stresses, such that turbulence reaches a twocomponent limit in the vicinity of the surface. In the case of a no-slip wall, one of the main difficulties lies in the particular scales of this phenomenon, linked to the non-locality of the fluctuating pressure, at the origin of a sensitivity of turbulence to the blockage effect up to a distance to the wall much larger than the thickness of the viscous sublayer. The layer in which $\overline{v^{2}}$ is affected by this effect is called the source layer (Hunt and Graham, 1978; Calmet and Magnaudet, 2003), surface-influenced layer (Brumley and Jirka, 1987) or blockage layer (Campagne et al., 2009). In order to model this blockage effect, the correct reproduction of the near-wall balance between viscous diffusion and dissipation, Eq. (1), is not sufficient: the asymptotic behaviour of the velocity-pressure gradient correlation term must be accounted for. This is the main purpose of the Elliptic Relaxation (Durbin, 1991) and Elliptic Blending (Manceau and Hanjalić, 2002) methods, briefly described below. 
(iv) The presence of a wall induces an increase of pressure fluctuations, called the wall echo effect, which can be explained by considering the Poisson equation for the fluctuating pressure $\mathrm{p}$

$\nabla^{2} \mathrm{p}=-2 \rho \frac{\partial U_{i}}{\partial x_{j}} \frac{\partial u_{j}}{\partial x_{i}}-\rho \frac{\partial u_{i}}{\partial x_{j}} \frac{\partial u_{j}}{\partial x_{i}}+\rho \frac{\overline{\partial u_{i}}}{\partial x_{j}} \frac{\partial u_{j}}{\partial x_{i}} \equiv S_{p}$

in a semi-infinite domain bounded by a wall located at $y=0$. In order to separate viscous and non-viscous effects, the fluctuating pressure is split into $\mathrm{p}=p+p^{s}$, where $p^{s}$ is Stokes' pressure, defined as the solution of the problem

$\nabla^{2} p^{s}=0$

$\frac{\partial p^{s}}{\partial y}=\rho \nu \frac{\partial^{2} v}{\partial y^{2}}$

In contrast to the blockage and wall echo effects, the appearance of Stokes' pressure is due to viscosity, and as shown by Kim (1989), it is negligible. The remaining fluctuating pressure field $p=\mathrm{p}-p^{s}$ is also solution of the Poisson equation (2), but with the boundary condition

$\frac{\partial p}{\partial y}=0$

since the burden of the viscous boundary condition for $\mathrm{p}$ is entirely carried by Stokes' pressure (Eq. 4). The Green's function of the semi-infinite domain then writes

$G_{\Omega}\left(\mathbf{x}, \mathbf{x}^{\prime}\right)=-\frac{1}{4 \pi\left\|\mathbf{x}^{\prime}-\mathbf{x}\right\|}-\frac{1}{4 \pi\left\|\mathbf{x}^{\prime \bullet}-\mathbf{x}\right\|}$,

where $\mathrm{x}^{\prime}$ denotes the image of $\mathrm{x}^{\prime}$ for the reflection in the plane of the wall. The solution of the Poisson equation for $p$ with the boundary condition (5) is (Launder et al., 1975)

$$
p(\mathbf{x})=\underbrace{-\int_{\Omega} \frac{S_{p}\left(\mathbf{x}^{\prime}\right)}{4 \pi\left\|\mathbf{x}^{\prime}-\mathbf{x}\right\|} \mathrm{d} V\left(\mathbf{x}^{\prime}\right)}_{p^{0}(\mathbf{x})} \underbrace{-\int_{\Omega} \frac{S_{p}\left(\mathbf{x}^{\prime}\right)}{4 \pi\left\|\mathbf{x}^{\prime \bullet}-\mathbf{x}\right\|} \mathrm{d} V\left(\mathbf{x}^{\prime}\right)}_{p^{I}(\mathbf{x})} .
$$

The fluctuating pressure $p$ is thus composed of two parts, $p^{0}$ and $p^{I}$. The first component, $p^{0}$, is the direct contribution of the flow in the domain $\Omega$. The image component, $p^{I}$, is the consequence of the reflection (echo) from the wall of the pressure waves (that propagate at infinite speed in the limit of incompressible fluids) and can be also regarded as the contribution of the mirrored flow.

In RANS modelling, the wall echo effect specifically denotes the consequence of the existence of this echo for the velocity-pressure gradient correlation involved in the Reynolds-stress transport equations,

$\phi_{i j}^{*}=-\frac{1}{\rho} \overline{u_{i} \frac{\partial p}{\partial x_{j}}}-\frac{1}{\rho} \overline{u_{j} \frac{\partial p}{\partial x_{i}}}$,

which can be decomposed into a principal term $\phi_{i j}^{* 0}$ and an image term $\phi_{i j}^{* I}$, in order to distinguish the contributions due to $p^{0}$ and $p^{I}$,

$$
\begin{aligned}
& \phi_{i j}^{* 0}(\mathbf{x})=-\frac{1}{\rho} \overline{u_{i} \frac{\partial p^{0}}{\partial x_{j}}}-\frac{1}{\rho} \overline{u_{j} \frac{\partial p^{0}}{\partial x_{i}}} \quad \text { and } \\
& \phi_{i j}^{* I}(\mathbf{x})=-\frac{1}{\rho} \overline{u_{i} \frac{\partial p^{I}}{\partial x_{j}}}-\frac{1}{\rho} \overline{u_{j} \frac{\partial p^{I}}{\partial x_{i}}},
\end{aligned}
$$

such that

$$
\begin{aligned}
\phi_{i j}^{*}(\mathbf{x})= & \underbrace{-\frac{1}{\rho} \int_{\Omega} \frac{\Psi_{i j}\left(\mathbf{x}, \mathbf{x}^{\prime}\right)}{4 \pi\left\|\mathbf{x}^{\prime}-\mathbf{x}\right\|} \mathrm{d} V\left(\mathbf{x}^{\prime}\right)}_{\phi_{i j}^{* 0}} \\
& \underbrace{-\frac{1}{\rho} \int_{\Omega} \frac{\Psi_{i j}\left(\mathbf{x}, \mathbf{x}^{\prime}\right)}{4 \pi\left\|\mathbf{x}^{\prime \bullet}-\mathbf{x}\right\|} \mathrm{d} V\left(\mathbf{x}^{\prime}\right)}_{\phi_{i j}^{* I}},
\end{aligned}
$$

where

$$
\Psi_{i j}\left(\mathbf{x}, \mathbf{x}^{\prime}\right)=\overline{u_{i}(\mathbf{x}) \frac{\partial S_{p}}{\partial x_{j}}\left(\mathbf{x}^{\prime}\right)}+\overline{u_{j}(\mathbf{x}) \frac{\partial S_{p}}{\partial x_{i}}\left(\mathbf{x}^{\prime}\right)}
$$

A clear distinction between the wall echo and the wall blockage effects is now possible: the wall echo effect corresponds to the appearance of the image term in the Green's function of the Poisson equation for the fluctuating pressure; the blockage effect corresponds to the modification of the velocity field due to the presence of the wall, which in turn induces a modification of $\Psi_{i j}\left(\mathbf{x}, \mathbf{x}^{\prime}\right)$. Consequently, the image terms, $p^{I}$ and $\phi_{i j}^{* I}$, are influenced by the blockage effect, and, conversely, the wall echo, through the modification of the pressure field, affects the velocity field, and, indirectly, $p^{0}$ and $\phi_{i j}^{* 0}$. Therefore, although two different mechanisms can be distinguished, they are always coupled. In terms of modelling, it is important to remark that the two effects have opposite influences on $\phi_{i j}^{*}$ in most of the near-wall region, in particular for its redistributive part. Indeed, the analysis of the asymptotic behaviour of the Reynolds stresses (see Appendix A) shows that the wall blockage leads to a strong damping of the redistribution (Manceau and Hanjalić, 2002). The blockage effect is so strong that, in a very thin vicinity of the wall, energy is redistributed from $\overline{v^{2}}$ to the tangential components, i.e., in a direction opposite to that observed in the rest of the boundary layer (Mansour et al., 1988). In contrast, the image term $\phi_{i j}^{* I}$ is of the same sign as $\phi_{i j}^{* 0}$, such that, outside the very thin region where $\phi_{i j}^{* 0}<0$, it increases the redistribution of energy from the streamwise component $\overline{u^{2}}$ to the wall-normal component $\overline{v^{2}}$, as pointed out by Manceau et al. (2001). 
The main objective of Elliptic Relaxation and Elliptic Blending approaches is to account for the influence of the wall blockage on the energy redistribution towards the wall-normal component, which is necessary to reproduce the two-component limit of turbulence.

\section{Elliptic Relaxation}

\subsection{Physical basis of the elliptic operator}

In the simplified case of turbulence in an unbounded domain, the image term in Eq. (10) vanishes and the velocitypressure gradient correlation reduces to $\phi_{i j}^{* 0}$. Durbin (1991) proposed to model the two-point correlation $\Psi_{i j}\left(\mathbf{x}, \mathbf{x}^{\prime}\right)$ that appears in Eq. (10), assuming an exponential decrease of the correlation under the form

$\frac{\Psi_{i j}\left(\mathbf{x}, \mathbf{x}^{\prime}\right)}{\lambda(\mathbf{x})}=\frac{\Psi_{i j}\left(\mathbf{x}^{\prime}, \mathbf{x}^{\prime}\right)}{\lambda\left(\mathbf{x}^{\prime}\right)} \exp \left(-\frac{\left\|\mathbf{x}^{\prime}-\mathbf{x}\right\|}{L}\right)$,

where $L$ is a characteristic length scale of this particular correlation. The validity of this hypothesis was confirmed by Manceau et al. (2001) using the DNS database of Moser et al. (1999). The scaling function $\lambda$ is introduced here to anticipate the fact that such a scaling will be necessary in the presence of a wall. The introduction of Eq. (12) in Eq. (10) yields

$\frac{\phi_{i j}^{*}(\mathbf{x})}{\lambda(\mathbf{x})}=-\frac{1}{\rho} \int \frac{\Psi_{i j}\left(\mathbf{x}^{\prime}, \mathbf{x}^{\prime}\right)}{\lambda\left(\mathbf{x}^{\prime}\right)} \frac{\exp \left(-\frac{\left\|\mathbf{x}^{\prime}-\mathbf{x}\right\|}{L}\right)}{4 \pi\left\|\mathbf{x}^{\prime}-\mathbf{x}\right\|} \mathrm{d} V\left(\mathbf{x}^{\prime}\right)$,

in which the Yukawa potential (Yukawa, 1935)

$\mathcal{G}_{\mathbb{R}^{3}}\left(\mathbf{x}, \mathbf{x}^{\prime}\right)=\frac{\exp \left(\frac{\left\|\mathbf{x}^{\prime}-\mathbf{x}\right\|}{L}\right)}{4 \pi\left\|\mathbf{x}^{\prime}-\mathbf{x}\right\|}$

appears, which is the Green's function associated with the elliptic operator

$\left(\frac{1}{L^{2}}-\nabla^{2}\right)$

i.e., the multiplicative inverse of the generalized function $\left(\frac{1}{L^{2}}-\nabla^{2}\right) \delta$ for the convolution product, where $\delta$ denotes the Dirac delta function. Therefore, Eq. (13) is the solution of the Elliptic Relaxation equation

$\left(\frac{1}{L^{2}}-\nabla^{2}\right) \frac{\phi_{i j}^{*}(\mathbf{x})}{\lambda(\mathbf{x})}=-\frac{\Psi_{i j}(\mathbf{x}, \mathbf{x})}{\rho \lambda(\mathbf{x})}$.

In the general case of inhomogeneous turbulence, $L$ is not constant in space, such that Eq. (13) is only an approximation of the solution of Eq. (16). Manceau and Hanjalić (2000) showed that this inversion error can be accounted for by modifying the elliptic operator (15).

In weakly inhomogeneous flows, the term $\Psi_{i j}\left(\mathbf{x}^{\prime}, \mathbf{x}^{\prime}\right) / \lambda\left(\mathbf{x}^{\prime}\right)$ can be considered constant in the sphere of influence of the Green's function and can be taken outside the integral, such that

$\Psi_{i j}(\mathbf{x}, \mathbf{x})=-\frac{\rho}{L^{2}} \phi_{i j}^{*}(\mathbf{x})$ is obtained. Therefore, Durbin (1991) assumed that the right hand side of Eq. (16) can be represented by any of the standard models $\phi_{i j}^{h}$ for the redistribution term, such as the SSG (Speziale et al., 1991) or the LRR (Launder et al., 1975) models, among others, which are valid for weakly inhomogeneous flows only, i.e., not in the viscous and buffer sublayers (Bradshaw et al., 1987). The Elliptic Relaxation equation then becomes

$$
\left(1-L^{2} \nabla^{2}\right) \frac{\phi_{i j}^{*}}{\lambda}=\frac{\phi_{i j}^{h}}{\lambda}
$$

These models $\phi_{i j}^{h}$ represent the pressure-strain correlation $\phi_{i j}$ rather than the velocity-pressure gradient $\phi_{i j}^{*}$, which implies that the pressure diffusion $D_{i j}^{p}=\phi_{i j}^{*}-\phi_{i j}$ is considered negligible in weakly inhomogeneous regions, or, alternatively, is included in the model for the triple correlation (turbulent diffusion). It is important to emphasize the fact that this hypothesis is not used in near-wall regions, since the boundary conditions of Eq. (18) will be explicitly formulated in order to satisfy the asymptotic behaviour of $\phi_{i j}^{*}$ that includes pressure diffusion, which is crucial to reproduce the near-wall budgets of the Reynolds stresses (Appendix A) and consequently the two-component limit of turbulence.

The main characteristic of this approach, compared to standard models based on algebraic relations, is that $\phi_{i j}^{*}$ is the solution of a differential equation, which preserves the non-local character of $\phi_{i j}^{*}$ inherited from the Poisson equation for the fluctuating pressure, and, additionally, makes the enforcement of the asymptotic behaviour of $\phi_{i j}^{*}$ in the vicinity of the wall possible, by means of the boundary conditions of Eq. (18). $\phi_{i j}^{*}$ is thus constrained to fulfill the correct near-wall behaviour, and is relaxed by the elliptic operator toward the standard model $\phi_{i j}^{h}$ used in the right hand side of Eq. (18) far from the wall, hence the name Elliptic Relaxation. The rapidity of the migration from the near-wall to the weakly inhomogeneous behaviour is driven by the length scale $L$. The price to pay is one additional equation for each of the independent components of the $\phi_{i j}^{*}$ tensor, leading to a 13-equation model.

In the case of a semi-infinite domain $\Omega$, bounded by a wall at $y=0$, similar to the case of the Poisson equation for the fluctuating pressure, the solution of Eq. (16) involves, in addition to the term in Eq. (13), the image term

$\frac{1}{\rho} \int_{\Omega} \frac{\Psi_{i j}\left(\mathbf{x}^{\prime}, \mathbf{x}^{\prime}\right)}{\lambda\left(\mathbf{x}^{\prime}\right)} \frac{\exp \left(-\frac{\left\|\mathbf{x}^{\prime \bullet}-\mathbf{x}\right\|}{L}\right)}{4 \pi\left\|\mathbf{x}^{\prime \bullet}-\mathbf{x}\right\|} \mathrm{d} V\left(\mathbf{x}^{\prime}\right)$.

A term similar to the image term $\phi_{i j}^{* I} / \lambda$ is thus obtained by solving the Elliptic Relaxation equation in a wall-bounded domain, but this term is not the same as that given by the introduction of the hypothesis (12) in Eq. (10) (the exponential is evaluated in $\mathbf{x}^{\prime \bullet}$ instead of $\mathbf{x}^{\prime}$ ). This discrepancy does not lead to any limitation of the model, since the asymptotic behaviour of the Reynolds stress tensor $\overline{u_{i} u_{j}}$ is not affected. 
Table 1: Wall boundary conditions for the components of the tensor $\mathcal{F}_{i j}=\phi_{i j}^{*} / \lambda$, for $\lambda=k$ and $\lambda=k \varepsilon$. A $w$ index or exponent denotes a value at the wall.

\begin{tabular}{ccccccc}
\hline & $\mathcal{F}_{11}^{w}$ & $\mathcal{F}_{22}^{w}$ & $\mathcal{F}_{33}^{w}$ & $\mathcal{F}_{12}^{w}$ & $\mathcal{F}_{13}^{w}$ & $\mathcal{F}_{23}^{w}$ \\
\hline$\lambda=k$ & $-\frac{1}{2} \mathcal{F}_{22}^{w}$ & $-\frac{20 \nu^{2}}{\varepsilon_{w}} \lim _{y \rightarrow 0} \frac{\overline{v^{2}}}{y^{4}}$ & $-\frac{1}{2} \mathcal{F}_{22}^{w}$ & $-\frac{20 \nu^{2}}{\varepsilon_{w}} \lim _{y \rightarrow 0} \frac{\overline{u v}}{y^{4}}$ & 0 & $-\frac{20 \nu^{2}}{\varepsilon_{w}} \lim _{y \rightarrow 0} \frac{\overline{v w}}{y^{4}}$ \\
$\lambda=k \varepsilon$ & $-\frac{1}{2} \mathcal{F}_{22}^{w}$ & $-\frac{20 \nu^{2}}{\varepsilon_{w}^{2}} \lim _{y \rightarrow 0} \frac{\overline{v^{2}}}{y^{4}}$ & $-\frac{1}{2} \mathcal{F}_{22}^{w}$ & $-\frac{20 \nu^{2}}{\varepsilon_{w}^{2}} \lim _{y \rightarrow 0} \frac{\overline{u v}}{y^{4}}$ & 0 & $-\frac{20 \nu^{2}}{\varepsilon_{w}^{2}} \lim _{y \rightarrow 0} \frac{\overline{v w}}{y^{4}}$ \\
\hline
\end{tabular}

\subsection{Near-wall behaviour}

In the vicinity of the wall, dominant terms in the Reynolds stress budget are viscous diffusion $\nu \partial^{2} \overline{u_{i} u_{j}} / \partial y^{2}$, dissipation $\varepsilon_{i j}$ and the velocity-pressure gradient correlation $\phi_{i j}^{*}$ (see Appendix A) such that the transport equation for $\overline{u_{i} u_{j}}$ reduces, at leading order, to

$\phi_{i j}^{*}-\varepsilon_{i j}=-\nu \frac{\partial^{2} \overline{u_{i} u_{j}}}{\partial y^{2}}$.

Introducing the asymptotic behaviour of the right hand side (Manceau and Hanjalić, 2002) leads to

$\phi_{i j}^{*}-\varepsilon_{i j}=-n(n+1) \lim _{y \rightarrow 0} \nu \frac{\overline{u_{i} u_{j}}}{y^{2}}$,

where $n=1$ for components only involving tangential velocities $\left(\overline{u^{2}}, \overline{w^{2}}\right.$ and $\left.\overline{u w}\right), n=2$ for $\overline{u v}$ and $\overline{v w}$, and $n=3$ for $\overline{v^{2}}$. The solution of Eq. (20) with boundary conditions $\overline{u_{i} u_{j}}=0$ leads to a behaviour of $\overline{u_{i} u_{j}}$ in $y^{n+1}$, such that reproducing the wall-limiting behaviour of $\overline{u_{i} u_{j}}$ is linked to the asymptotically correct modelling of the difference $\phi_{i j}^{*}-\varepsilon_{i j}$ in this region.

Since, as shown in Appendix A, $\phi_{i j}^{*}$ is negligible compared to $\varepsilon_{i j}$ for the components $\overline{u^{2}}, \overline{w^{2}}$ and $\overline{u w}$, a useful strategy consists in:

(i) choosing a simple model for $\varepsilon_{i j}$ that satisfies the correct asymptotic behaviour of these three components, regardless of the others;

(ii) choosing a model for $\phi_{i j}^{*}$ in such a way that $\phi_{i j}^{*}-\varepsilon_{i j}$ satisfies the asymptotic behaviour of the remaining components $\overline{u v}, \overline{v w}$ and $\overline{v^{2}}$, making sure that $\phi_{i j}^{*}$ is negligible compared to $\varepsilon_{i j}$ for the components $\overline{u^{2}}, \overline{w^{2}}$ and $\overline{u w}$.

In other terms, the model for $\varepsilon_{i j}$ is corrected by means of a choice for $\phi_{i j}^{*}$ that enforces the behaviour of the difference $\phi_{i j}^{*}-\varepsilon_{i j}$.

The way to fulfill these requirements is not unique, and a simple possibility is to use Rotta's model for the dissipation tensor

$\varepsilon_{i j}=\frac{\overline{u_{i} u_{j}}}{k} \varepsilon$.

This model indeed reproduces the behaviour (21) for the case $n=1$, provided that the correct wall boundary condition

$\varepsilon_{w}=2 \nu \lim _{y \rightarrow 0} \frac{k}{y^{2}}$ is imposed for the dissipation rate $\varepsilon$, where $k$ is the turbulent energy. In order to impose the wall-limiting behaviour of $\phi_{i j}^{*}$, it can be shown (Durbin, 1991) that the scaling function $\lambda$ in Eq. (12) must go to zero as $y^{2}$ at the wall, in such a way as to obtain finite (non-zero) boundary conditions for the tensor $\mathcal{F}_{i j}=\phi_{i j}^{*} / \lambda$ for the components $\overline{u v}, \overline{v w}$ and $\overline{v^{2}}$. Durbin (1991) uses the turbulent energy as the scaling function $(\lambda=k)$, a choice leading to the boundary conditions $\mathcal{F}_{i j}^{w}=\lim _{y \rightarrow 0} \phi_{i j}^{*} / \lambda$ given in table 1 . It is worth emphasizing that there are degrees of freedom for the choice of the boundary conditions for $\mathcal{F}_{11}, \mathcal{F}_{33}$ and $\mathcal{F}_{13}$, in so far as $\phi_{i j}^{*}$ is negligible in the budget of the corresponding Reynolds stresses. Therefore, the asymptotic behaviour of $\phi_{i j}^{*}=\lambda \mathcal{F}_{i j}$ is not crucial, provided that this term remains negligible compared to $\varepsilon_{i j}$. A simple and numerically robust choice is $\mathcal{F}_{11}^{w}=\mathcal{F}_{33}^{w}=\mathcal{F}_{13}^{w}=0$, but most of the authors use $\mathcal{F}_{11}^{w}=\mathcal{F}_{33}^{w}=-\frac{1}{2} \mathcal{F}_{22}^{w}$ and $\mathcal{F}_{13}^{w}=0$, in such a way that $\phi_{i j}^{*}$ is traceless. There is no theoretical argument in favour of this particular choice, since, as stated above, the model represents the velocity-pressure gradient correlation that is not traceless, but rather a practical argument: the Reynolds stress transport equation thus contracts to the standard $k$ equation, which avoids the necessity of modifying the standard coefficients of the $\varepsilon$-equation.

Manceau and Hanjalić (2002) pointed out that the boundary conditions $\mathcal{F}_{12}^{w}$ and $\mathcal{F}_{23}^{w}$ do not lead to the correct asymptotic behaviour in $y^{3}$ of $\overline{u v}$ and $\overline{v w}$, but rather in $y^{4}$. The reason for this choice is that no boundary condition can ensure a behaviour in $y^{3}$, and that $y^{4}$ is preferable to $y^{2}$ in order that the turbulent shear stress remains negligible in the budget.

Finally, Manceau et al. (2002) showed that the scaling function $\lambda=k \varepsilon$ is preferable in order to avoid the spurious amplification of redistribution in the logarithmic layer observed with Durbin's model (Wizman et al., 1996; Manceau and Hanjalić, 2000). In this case, boundary conditions for $\mathcal{F}_{i j}$ are slightly modified, as shown in table 1 .

\subsection{Elliptic Relaxation for the dissipation tensor}

Model (22) is not valid in regions far from the wall, since dissipative scales are much more isotropic than energetic scales, due to scale separation. Therefore, it is desirable that the dissipation tensor tend to isotropy far from the wall. Durbin (1991) proposed to decompose dissipation into two terms,

$\varepsilon_{i j}=\frac{\overline{u_{i} u_{j}}}{k} \varepsilon+\xi_{i j}$,

where $\xi_{i j}$ is merely the difference between the dissipation tensor and Rotta's model (22), and to solve an Elliptic Relaxation equation for $\xi_{i j}$ of the same form as Eq. (18):

$\left(1-L^{2} \nabla^{2}\right) \frac{\xi_{i j}}{\lambda}=\frac{\xi_{i j}^{h}}{\lambda}$,

where

$\xi_{i j}^{h}=\frac{2}{3} \varepsilon \delta_{i j}-\frac{\overline{u_{i} u_{j}}}{k} \varepsilon$, 
with the boundary condition $\xi_{i j}=0$ at the wall. Consequently, close to the wall, $\xi_{i j} \rightarrow 0$, such that

$\varepsilon_{i j}=\frac{\overline{u_{i} u_{j}}}{k} \varepsilon+\xi_{i j} \rightarrow \frac{\overline{u_{i} u_{j}}}{k} \varepsilon$,

whereas, far from the wall, $\xi_{i j} \rightarrow \xi_{i j}^{h}$ such that

$\varepsilon_{i j} \rightarrow \frac{\overline{u_{i} u_{j}}}{k} \varepsilon+\xi_{i j}^{h}=\frac{2}{3} \varepsilon \delta_{i j}$.

In contrast with $\phi_{i j}^{*}$, there is no theoretical foundation for the use of an Elliptic Relaxation operator for $\varepsilon_{i j}$. However, a velocity gradient-pressure gradient correlation appears in the exact transport equation for $\varepsilon_{i j}$ (Durbin and Speziale, 1991; Speziale and Gatski, 1997), which shows that dissipation also inherits non-locality from the fluctuating pressure. Manceau and Hanjalić (2002) observed, from the DNS database of Moser et al. (1999), that the region where the dissipation tensor cannot be modelled as isotropic is much larger than the viscous sublayer and comparable to the blockage layer, which justifies the use of elliptic relaxation for this tensor. Nonetheless, directly applying the elliptic operator to $\varepsilon_{i j}$, rather than to a term of its transport equation, is a strong hypothesis, equivalent to applying this operator directly to $\overline{u_{i} u_{j}}$ rather than $\phi_{i j}^{*}$.

Using the linearity of the elliptic operator, separately solving Eqs. (18) and (25) is not necessary. Since only the difference $\phi_{i j}^{*}-\varepsilon_{i j}$ enters the Reynolds stress transport equation, the following equation is solved:

$f_{i j}-L^{2} \nabla^{2} f_{i j}=f_{i j}^{h}=\frac{1}{\lambda}\left(\phi_{i j}^{h}-\frac{2}{3} \varepsilon \delta_{i j}+\frac{\overline{u_{i} u_{j}}}{k} \varepsilon\right)$,

where $f_{i j}=\left(\phi_{i j}^{*}-\xi_{i j}\right) / \lambda$. The boundary conditions described in table 1 remain valid for $f_{i j}$.

\section{Elliptic Blending}

The popularity of the Elliptic Relaxation Reynolds stress models is limited by the 6 additional differential equations to solve in addition to the 7 equations of standard models, and by the numerical instabilities due to the wall boundary conditions.

The main feature of Elliptic Relaxation, the reproduction of the blockage effect, is due to the fact that the elliptic operator ensures a smooth relaxation from the correct asymptotic behaviour of $\phi_{i j}^{*}$ imposed by the boundary conditions to the standard model $\phi_{i j}^{h}$. However, it can be noted that this transition, for the 6 components of $\phi_{i j}^{*}$, is driven by the same operator, with a single length scale $L$ (although Manceau et al., 2001, showed that different length scales can be introduced to improve the accuracy of the representation of the physics, such a refinement is generally not considered necessary). In order to exploit this redundancy, Manceau and Hanjalić (2002) proposed the Elliptic Blending approach, for the purpose of building a model preserving the desirable features of Elliptic Relaxation, together with a reduced number of equations and an improved numerical stability.

\subsection{Imposing the near-wall behaviour}

The method consists in ensuring the transition from the near-wall to the weakly inhomogeneous behaviour using a single scalar function $f_{\phi}$, which must tend to 0 at the wall and to 1 far from the wall, under the form

$\phi_{i j}^{*}=\left(1-f_{\phi}\right) \phi_{i j}^{w}+f_{\phi} \phi_{i j}^{h}$,

where $\phi_{i j}^{h}$ is a standard model. The near-wall form of the velocity-pressure gradient term $\phi_{i j}^{w}$ and the blending function $f_{\phi}$ are modelled in order to replicate, as closely as possible, the features of the Elliptic Relaxation model (18).

To this aim, the elliptic relaxation effect is transposed to the blending function $f_{\phi}$, using the elliptic equation

$\left(1-L^{2} \nabla^{2}\right) \frac{f_{\phi}}{\lambda}=\frac{f_{\phi}^{h}}{\lambda}=\frac{1}{\lambda}$.

Introducing the function $\alpha=f_{\phi} / \lambda$, we have

$$
\alpha-L^{2} \nabla^{2} \alpha=\frac{1}{\lambda}
$$

with the boundary condition $\alpha=0$ at the wall. The solution of this equation goes to zero at the wall and to one far from the wall, thus providing the appropriate blending between the two formulations $\phi_{i j}^{w}$ and $\phi_{i j}^{h}$. The thickness of the region of influence of the near-wall model is driven by the length scale $L$. Following the proposal of Durbin (1991) for the Elliptic Relaxation strategy, validated by Manceau et al. (2001) using DNS data, this length scale is modelled as

$L=C_{L} \max \left(\frac{k^{3 / 2}}{\varepsilon}, C_{\eta} \frac{\nu^{3 / 4}}{\varepsilon^{1 / 4}}\right)$.

Using a near-wall model $\phi_{i j}^{w}$ based on the asymptotic behaviour of $\phi_{i j}^{*}$ at the wall, the method thus provides a way to extend the validity of any standard model $\phi_{i j}^{h}$ to the near-wall region. Although any model can be used for $\phi_{i j}^{h}$, the discussion and results below will be restricted to the case of the SSG model (Speziale et al., 1991)

$$
\begin{aligned}
\phi_{i j}^{h}= & -\left(g_{1}+g_{1}^{*} \frac{P}{\varepsilon}\right) \varepsilon b_{i j}+g_{2} \varepsilon\left(b_{i k} b_{k j}-\frac{1}{3} b_{k l} b_{k l} \delta_{i j}\right) \\
& +\left(g_{3}-g_{3}^{*} \sqrt{b_{k l} b_{k l}}\right) k S_{i j} \\
& +g_{4} k\left(b_{i k} S_{j k}+b_{j k} S_{i k}-\frac{2}{3} b_{l m} S_{l m} \delta_{i j}\right) \\
& +g_{5} k\left(b_{i k} W_{j k}+b_{j k} W_{i k}\right) .
\end{aligned}
$$

Note that, in this model, the term tensorially nonlinear in $b_{i j}$ was usually discarded $\left(g_{2}=0\right)$ in the past to improve numerical robustness (Manceau, 2003, 2005). In order to investigate the influence if this term in channel flows for a wide range of Reynolds numbers, computations are performed with a simple, finite-difference, 1D code. The grid, refined in order to reach a fully converged solution at $R e_{\tau}=4200$, consists of 400 points with near-wall clustering, such that the first grid point next to the wall is located at about $y^{+}=0.3$ for $R e_{\tau}=4200$ and down to $y^{+}=0.01$ for $R e_{\tau}=180$, although, as mentioned by Manceau and 
Hanjalić (2002), the model is not very sensitive to a coarsening of the near-wall mesh, as long as the first point is located in the viscous sublayer $\left(y^{+}<5\right)$.

Throughout the present article, the two coefficients $C_{L}$ and $A_{1}$ introduced in the equations by the Elliptic Blending method (see Appendix C) are calibrated, for each formulation, in order to reproduce as closely as possible channel flows at friction Reynolds numbers $R e_{\tau}$ ranging from 180 to 4200 , by comparison with available channel flow DNS data (Moser et al., 1999; Lozano-Durán and Jiménez, 2014), attaching more importance to high-Reynolds numbers. Indeed, it must be emphasized that the present model is, in the strict sense of the terms, a near-wall model, not a low-Reynolds number model. Viscous effects and non-viscous effects described in section 2 are accounted for in the near-wall region, i.e., in the region where $f_{\phi}$ is significantly lower that one, but not further from the wall, where it completely relies on the weakly inhomogeneous model $\phi_{i j}^{h}$. Therefore, the effects of viscosity in low-Reynolds number regions, outside the region where $\phi_{i j}^{w}$ is active, are not accounted for. Consequently, in the calibration process, a particular attention is paid to the reproduction of high Reynolds number cases. Other coefficients are the same for all the versions of the EB-RSM used in the present paper, and are listed in Appendix C.

Fig. 1a shows that the influence of this term is weak for the case of channel flows. However, since this term is necessary to reproduce the final stage of the decay of grid turbulence (Sarkar and Speziale, 1990), it could be easily reintroduced if necessary, with specific $C_{L}$ and $A_{1}$ coefficients, given in Appendix C. In the rest of this article, the recommended formulation of the EB-RSM is denoted as the reference model: in this model, the coefficient $g_{2}$ is zero.

Similar to $\phi_{i j}^{*}$, the near-wall behaviour of the dissipation tensor is imposed by using a blending function $f_{\varepsilon}$,

$$
\begin{aligned}
\varepsilon_{i j} & =\left(1-f_{\varepsilon}\right) \varepsilon_{i j}^{w}+f_{\varepsilon} \varepsilon_{i j}^{h} \\
& =\left(1-f_{\varepsilon}\right) \frac{\overline{u_{i} u_{j}}}{k} \varepsilon+f_{\varepsilon} \frac{2}{3} \varepsilon \delta_{i j} .
\end{aligned}
$$

From the original article of Manceau and Hanjalić (2002) to the recent paper of Manceau et al. (2014), several expressions for the two blending functions $f_{\phi}$ and $f_{\varepsilon}$ were used. Section 4.2 provides some clarifications about the reasons for these choices, and compares the different versions.

In order to reproduce the same behaviour as the Elliptic Relaxation model, $\phi_{i j}^{w}$ must reproduce the same walllimiting values as given by table 1 , i.e., in the case of a wall in $y=0$, the model must be of the form

$\phi_{i j}^{w}=-\frac{\varepsilon}{k}\left(\begin{array}{ccc}-\frac{5}{2} \overline{v^{2}} & 5 \overline{u v} & 0 \\ 5 \overline{u v} & 5 \overline{v^{2}} & 5 \overline{v w} \\ 0 & 5 \overline{v w} & -\frac{5}{2} \overline{v^{2}}\end{array}\right)$.

It is important to emphasize that table 1 provides boundary conditions to the Elliptic Relaxation model, which can always be written in the coordinate frame aligned with the wall. The implementation in a CFD code is then based on a local change of coordinate frame to compute the values of the Dirichlet boundary conditions for the 6 components of $f_{i j}$. In contrast, in the case of the Elliptic Blending model, we are not talking about boundary conditions for a tensorial differential equation, but about an algebraic expression for the near-wall tensor $\phi_{i j}^{w}$. Therefore, a general, objective, expression for $\phi_{i j}^{w}$ is sought, independent of the relative orientation of the wall and the coordinate frame, that reduces to Eq. (36) in the case of a wall located at $y=0$. Here, the decisive feature of the approach is the availability of the scalar $\alpha$, solution of Eq. (32). Indeed, since the boundary condition is $\alpha=0$, the wall is an isocontour of $\alpha$, such that the gradient of $\alpha$ can be used to identify the wall-normal direction. Therefore, the unit vector

$\mathbf{n}=\frac{\nabla \alpha}{\|\nabla \alpha\|}$

is introduced. Manceau and Hanjalić (2002) empirically built the model

$$
\begin{aligned}
\phi_{i j}^{w}= & -5 \frac{\varepsilon}{k}\left(\overline{u_{i} u_{k}} n_{j} n_{k}+\overline{u_{j} u_{k}} n_{i} n_{k}\right. \\
& \left.-\frac{1}{2} \overline{u_{k} u_{l}} n_{k} n_{l} n_{i} n_{j}-\frac{1}{2} \overline{u_{k} u_{l}} n_{k} n_{l} \delta_{i j}\right)
\end{aligned}
$$

that satisfies Eq. (36). Here, a more systematic method for deriving the $\phi_{i j}^{w}$ model is proposed, in order to demonstrate the uniqueness of this solution.

In order for the tensor $\boldsymbol{\Phi}^{w}=\phi_{i j}^{w}$ to replicate the behaviour (36) for a wall at $y=0$, i.e., for $\left(n_{1}, n_{2}, n_{3}\right)=$ $(0,1,0)$, it must be a function of the variables explicitly involved in this equation. Specifically, an expression of the symmetric tensor $\boldsymbol{\Phi}^{w} / \varepsilon$ is sought as a function of one symmetric tensor $\mathbf{b}=b_{i j}=\overline{u_{i} u_{j}} /(2 k)-\delta_{i j} / 3$ (since, as mentioned above, $\boldsymbol{\Phi}^{w}$ is traceless, it is convenient, without limiting the generality of the analysis, to work with the turbulent anisotropy $\mathbf{b}$ ), and of the vector $\mathbf{n}$, in order to identify the wall-normal direction. There is no need to distinguish among the tangential directions, since Eq. (36) is invariant by rotation around the wall-normal vector. The theory of invariants (for details, see Appendix B) shows that such functions, invariant under the full orthogonal group of transformations, are linear combinations of the elements of the functional basis, which contains 5 irreducible terms,

$$
\begin{aligned}
& \frac{\boldsymbol{\Phi}^{w}}{\varepsilon}=\beta_{1} \mathbf{b}+\beta_{2} \mathbf{M}+\beta_{3}\left(\mathbf{b} \mathbf{M}+\mathbf{M b}-\frac{2}{3}\{\mathbf{b} \mathbf{M}\} \mathbf{I}\right) \\
& +\beta_{4}\left(\mathbf{b}^{2}-\frac{1}{3}\left\{\mathbf{b}^{2}\right\} \mathbf{I}\right)+\beta_{5}\left(\mathbf{b}^{2} \mathbf{M}+\mathbf{M} \mathbf{b}^{2}-\frac{2}{3}\left\{\mathbf{b}^{2} \mathbf{M}\right\} \mathbf{I}\right),
\end{aligned}
$$

where $\mathbf{I}=\delta_{i j}$ stands for the identity tensor, $\mathbf{M}=\mathbf{n} \otimes \mathbf{n}-$ $\frac{1}{3} \mathbf{I}$ and $\{$.$\} represents the trace. The coefficients \beta_{i}$ are polynomial functions of the terms of the integrity basis that consists of the four invariants $\left\{\mathbf{b}^{2}\right\},\left\{\mathbf{b}^{3}\right\},\{\mathbf{b} \mathbf{M}\}$ and $\left\{\mathbf{b}^{2} \mathbf{M}\right\}$. However, since Eq. (36) is linear in the Reynolds 


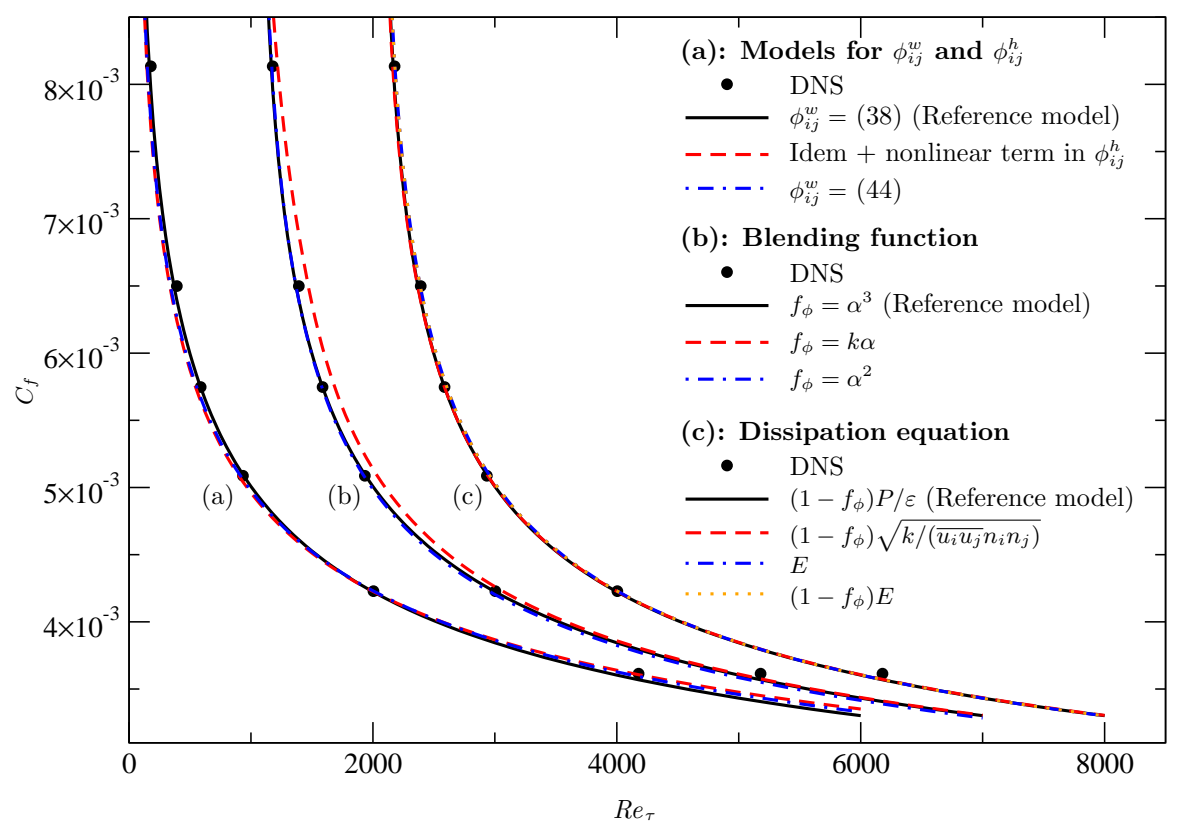

Figure 1: Comparison of various options for (a): the formulation of the models for $\phi_{i j}^{w}$ and $\phi_{i j}^{h}$ entering Eq. (30); (b): the blending function $f_{\phi}$ entering Eq. (30); (c) The additional production term in the $\varepsilon$-equation. Comparisons are performed in a channel flow for 90 different imposed values of $R e_{\tau}$ ranging from 100 to 6000. For the sake of clarity, curves are shifted to the right for comparisons (b) and (c).

stress tensor, terms nonlinear in $\mathbf{b}$ must be excluded from Eq. (39), which yields

$$
\begin{aligned}
\frac{\boldsymbol{\Phi}^{w}}{\varepsilon}= & a_{1} \mathbf{b}+a_{2}\left(1+a_{2}^{\prime}\{\mathbf{b} \mathbf{M}\}\right) \mathbf{M} \\
& +a_{3}\left(\mathbf{b} \mathbf{M}+\mathbf{M} \mathbf{b}-\frac{2}{3}\{\mathbf{b} \mathbf{M}\} \mathbf{I}\right),
\end{aligned}
$$

where $a_{1}, a_{2}, a_{2}^{\prime}$ and $a_{3}$ are constants. Writing Eq. (40) in the particular case of a wall normal vector $\left(n_{1}, n_{2}, n_{3}\right)=$ $(0,1,0)$ and comparing with Eq. (36), leads to a system of equations with the unique solution

$a_{1}=-\frac{20}{3} \quad ; \quad a_{2}=-5 \quad ; \quad a_{2}^{\prime}=-1 \quad ; \quad a_{3}=-10$.

Expanding this solution in terms of the Reynolds stress tensor and the wall normal vector yields Eq. (38). This model then replicates the near-wall behaviour of Durbin's Elliptic Relaxation model.

It is interesting to note that another solution can be obtained based on different hypotheses. It was pointed out above that Durbin's model is not able to reproduce the correct asymptotic behaviour in $y^{3}$ of the components $\overline{u v}$ and $\overline{v w}$. This is not the case with the Elliptic Blending model, since the model (40) with the coefficients

$a_{1}=-\frac{8}{3} \quad ; \quad a_{2}=-5 \quad ; \quad a_{2}^{\prime}=\frac{7}{5} \quad ; \quad a_{3}=-4$

gives the exact asymptotic behaviour

$\phi_{i j}^{w}=-\frac{\varepsilon}{k}\left(\begin{array}{ccc}-\frac{5}{2} \overline{v^{2}} & 2 \overline{u v} & 0 \\ 2 \overline{u v} & 5 \overline{v^{2}} & 2 \overline{v w} \\ 0 & 2 \overline{v w} & -\frac{5}{2} \overline{v^{2}}\end{array}\right)$, such that $\overline{u v}$ and $\overline{v w}$ now tend to zero as $y^{3}$ at the wall. The general expression, similar to Eq. (38), writes

$$
\begin{aligned}
\phi_{i j}^{w}= & -\frac{\varepsilon}{k}\left(2 \overline{u_{i} u_{k}} n_{j} n_{k}+2 \overline{u_{j} u_{k}} n_{i} n_{k}+\frac{7}{2} \overline{u_{k} u_{l}} n_{k} n_{l} n_{i} n_{j}\right. \\
& \left.-\frac{5}{2} \overline{u_{k} u_{l}} n_{k} n_{l} \delta_{i j}\right) .
\end{aligned}
$$

Manceau and Hanjalić (2002) identified this possibility, albeit only under the particular form (43). For the case of a channel flow, they reached the paradoxical conclusion that this formulation, although it satisfies the asymptotic behaviour of all the Reynolds stress components, gives results less satisfactory than formulation (38). Törnblom and Johansson (2007) empirically obtained Eq. (44), and applied it to the case of separation control in an asymmetric diffuser. Recently, Vanpouille (2013) compared the two versions and, for reasons similar to Manceau and Hanjalić (2002), recommends the use of Eq. (38).

Fig. 1a compares the friction coefficient $C_{f}=\tau_{w} /\left(\frac{1}{2} \rho U_{b}^{2}\right)$ predicted by the two formulations in a channel flow as a function of $R e_{\tau}$. It can be seen that the two formulations provide very similar predictions when properly calibrated. The reason for preferring Eq. (38), although it does not provide the exact asymptotic behaviour of $\overline{u v}$ and $\overline{v w}$, is that the budgets of these components are globally not correctly reproduced using Eq. (44), as shown in Fig. 2, in which the production $P_{12}$, velocity-pressure gradient correlation $\phi_{12}^{*}$, dissipation $\varepsilon_{12}$ and total diffusion (viscous + turbulent) $D_{12}$ terms are plotted. It can be seen that the amplitudes of these terms are strongly underestimated using formulation (44), in contrast with formulation (38) that satisfactorily reproduces the different terms, in particular $P_{12}$ and $\phi_{12}^{*}$. Fig. 3 shows that, in a very thin near- 

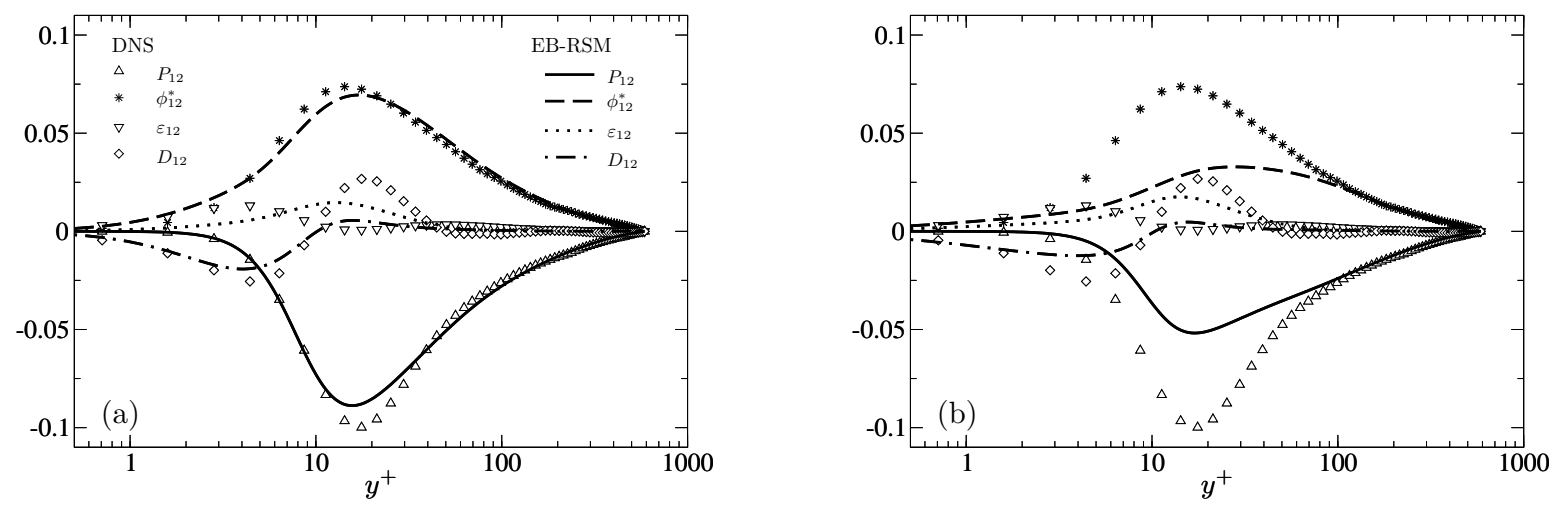

Figure 2: Computation of a channel flow at $R e_{\tau}=590$. Budget of the $\overline{u v}$ component. Comparison of the formulations (a) Eq. (38) and (b) Eq. (44).
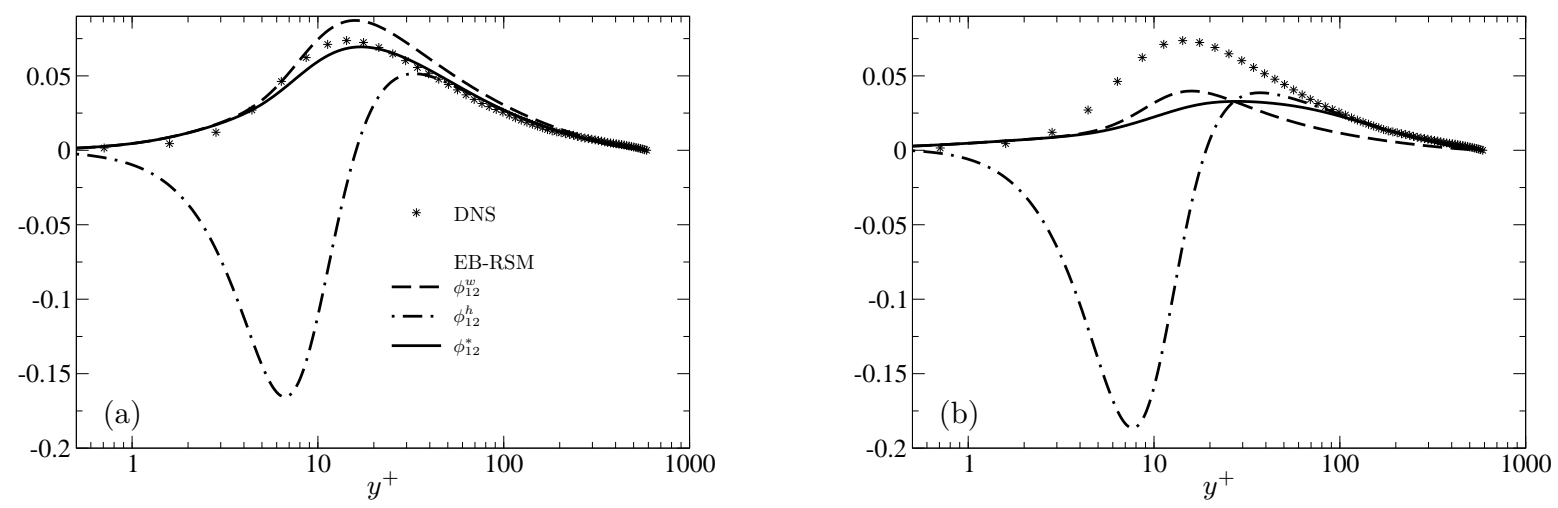

Figure 3: Computation of a channel flow at $R e_{\tau}=590$. Decomposition of $\phi_{12}^{*}$ into its near-wall and weakly inhomogeneous contributions. Comparison of the formulations (a) Eq. (38) and (b) Eq. (44).

wall region $\left(y^{+}<3\right)$, the asymptotically exact formulation (44) for $\phi_{12}^{w}$ indeed well reproduces the DNS data, but the blending with the $\phi_{12}^{h}$ model that is erroneous below $y^{+}=30$ yields a significant underestimation of $\phi_{12}^{*}$. Formulation (38), although it overestimates $\phi_{12}^{*}$ by a factor $5 / 2$ in the vicinity of the wall, provides a better prediction in the buffer layer, such that the blending with $\phi_{12}^{h}$ leads to an improved prediction. Therefore, although it is by compensation of errors, formulation (38) better represents the physical mechanisms in play in the $\overline{u v}$-equation, and it is retained in the reference model.

\subsection{Elliptic Blending functions}

In Eqs. (30) and (35), the blending functions $f_{\phi}$ and $f_{\varepsilon}$ must go to zero sufficiently fast at the wall in order to make sure that the weakly inhomogeneous terms $\phi_{i j}^{h}$ and $\varepsilon_{i j}^{h}$ do not perturb the behaviour of the near-wall terms $\phi_{i j}^{w}$ and $\varepsilon_{i j}^{w}$, respectively. The most critical case is that of the wall normal Reynolds stress $\overline{v^{2}}$ : since $\phi_{22}^{h}$ given by the SSG model (as other models) goes to a constant at the wall, and $\phi_{22}^{w}$ goes to zero as $y^{2}$, the blending function $f_{\phi}$ must go to zero at least as fast as $y^{2}$. The same holds for $f_{\varepsilon}$.

In the original Elliptic Blending model (Manceau and Hanjalić, 2002), the scaling function $\lambda$ in the equation (31) for the blending function $f_{\phi}$ was defined, similar to the case of the Elliptic Relaxation model (Durbin, 1991), as $\lambda=k$, such that $f_{\phi}=\lambda \alpha$ goes to zero as $y^{3}$. For $f_{\varepsilon}$, it was shown, using an analysis of a channel flow DNS database, that the length scale of the transition from the near-wall form to the isotropic form of $\varepsilon_{i j}$ is larger than for $\phi_{i j}^{*}$, which led to the introduction of the scaling function $\lambda=A k$ for $\varepsilon_{i j}$, where $A$ is Lumley's flatness parameter (Lumley, 1978).

However, this original formulation rapidly appeared numerically unstable for flows in complex geometries (Thielen et al., 2001, 2005) and was only applied in channel flows (Shin et al., 2003; Waclawczyk et al., 2004). These difficulties can be traced to the remaining nonlinearities, which are mainly due to Lumley's flatness parameter in $f_{\varepsilon}$. Moreover, it was observed that $f_{\phi}$ does not go properly to one when the flow is not sufficiently close to homogeneity: when spatial variations of $k$ are not small enough, the Laplacian in Eq. (31) is not negligible, such that $\alpha \neq 1 / k$. This behaviour is observed for instance in low-Reynoldsnumber channel flows, as shown in Fig. 4a. In this figure, the DNS data of Moser et al. (1999) are used to obtain the length scale from Eq. (33) and the turbulent kinetic energy $k$, which in turn are used to evaluate $\alpha$ from Eq. (32) and $f_{\phi}=k \alpha$. It is seen that the latter does not exactly go to one. Therefore, the model does not tend properly to 

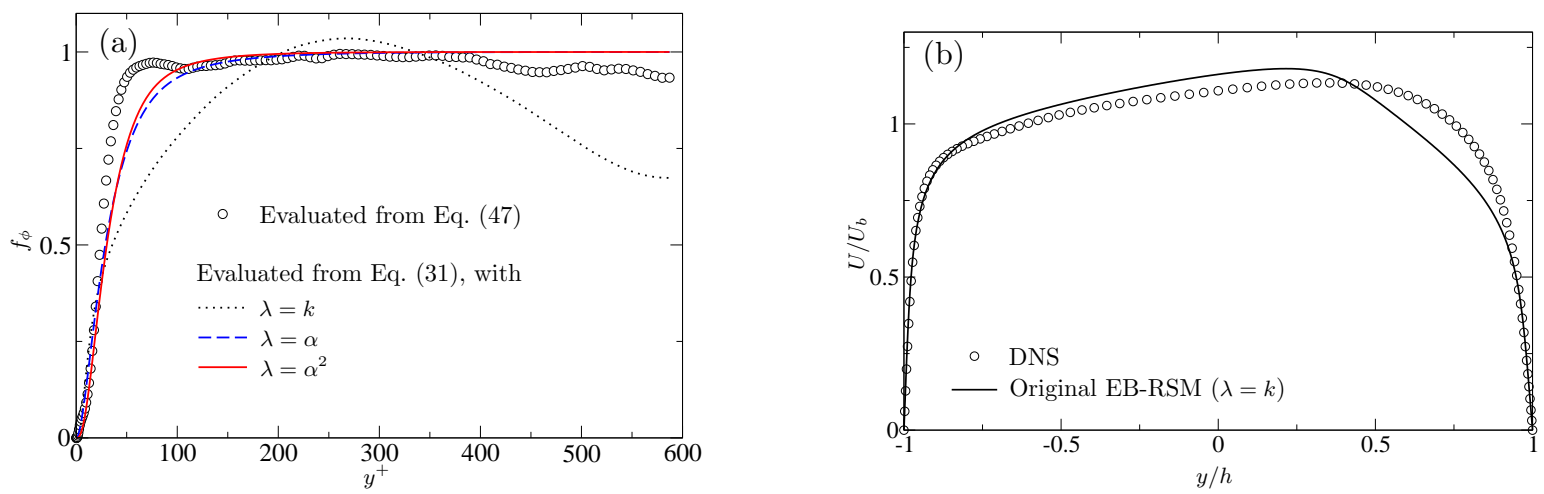

Figure 4: (a) Channel flow at $R e_{\tau}=590$. Comparison of the blending function $f_{\phi}$ evaluated a priori using DNS data (Moser et al., 1999) and obtained by solving the elliptic equation for $\alpha$ (with $L$ and $k$ from the DNS database). (b) Rotating channel flow at $R e=7000$ and $R o=1 / 6$. Comparison of the mean velocity profiles given by the highly-resolved LES of Lamballais et al. (1998) and by the original formulation of the EB-RSM (Manceau and Hanjalić, 2002).

the SSG model far from the wall, which is an undesired effect of the Elliptic Blending strategy. Finally, the model is erroneously sensitive to rotation, as shown in Fig. 4b, in comparison to the highly-resolved LES data of Lamballais et al. (1998) at $R e=h U_{b} / \nu=7000$ and $R o=2 \omega h / U_{b}=1 / 6$, where $h, U_{b}$ and $\omega$ are the half-width of the channel, the bulk velocity and the spanwise rotation rate, respectively (this test case is investigated in detail in section 5). The velocity profile predicted by the model behaves incorrectly in the region $0.3<y / h<0.9$, which leads to divergence of the computations for higher rotation numbers (see section $5)$.

Therefore, Manceau (2003) proposed an improved version, with $\lambda=\alpha$, which was successfully applied to many configurations (Manceau, 2005; Thielen et al., 2005; Borello et al., 2005; Choi et al., 2005; Viti et al., 2007; Shin et al., 2007; Ho et al., 2007; Fadai-Ghotbi et al., 2008; Choi and Kim, 2008a,b; Oceni et al., 2010; Terekhov and Pakhomov, 2011; Choi and Kim, 2012; Billard et al., 2012; Pakhomov and Terekhov, 2013a,b,c). More recently, Lecocq et al. (2008) proposed to use $\lambda=\alpha^{2}$, a version subsequently adopted by Dehoux et al. (2012), Benhamadouche et al. (2012) and Manceau et al. (2014), albeit with different coefficients, by Vanpouille (2013) for the derivation of an explicit algebraic model, and extended to hybrid RANS-LES by Fadai-Ghotbi et al. (2010a,b). The last two versions are actually close to each other and have shown equally good performance, of course with different coefficients. In association with several possibilities for the form of the dissipation equation $\varepsilon$, this leads to some variability of the model. The purpose of the present section is to compare these two formulations in order to be able to decide between them, and to fix the set of coefficients.

The rationale for using $\lambda=\alpha$ is to reproduce at best the asymptotic behaviour of the component normal to the wall $\phi_{22}^{*}$, at the origin of the two-component limit of turbulence, as shown in section 2. From Eq. (30), it can be deduced that the blending function must satisfy the fol- lowing relation:

$f_{\phi}=\frac{\phi_{22}^{*}-\phi_{22}^{w}}{\phi_{22}^{h}-\phi_{22}^{w}}$.

The asymptotic behaviour of the terms on the right hand side can be easily evaluated. Since $\phi_{22}^{*}$ and $\phi_{22}^{w}$ are both in $y^{2}$ and $\phi_{22}^{h}$ goes to a finite value at the wall, this relation suggests that $f_{\phi}$ must tend to zero as $y^{2}$, which is satisfied by $f_{\phi}=\alpha^{2}$, corresponding to a scaling function $\lambda=\alpha$. Unfortunately, this line of reasoning is correct only if the blending function $f_{\varepsilon}$ used for the dissipation term is different from $f_{\phi}$, which is not the case in the model proposed by Manceau (2003). Indeed, in the case $f_{\varepsilon}=f_{\phi}$, the two relations (30) and (35) can be formulated as a single relation for the difference $\phi_{i j}^{*}-\varepsilon_{i j}$,

$\phi_{i j}^{*}-\varepsilon_{i j}=\left(1-f_{\phi}\right)\left(\phi_{i j}^{w}-\varepsilon_{i j}^{w}\right)+f_{\phi}\left(\phi_{i j}^{h}-\varepsilon_{i j}^{h}\right)$.

For the term corresponding to the wall-normal direction, this relation now leads to

$f_{\phi}=\frac{\left(\phi_{22}^{*}-\varepsilon_{22}\right)-\left(\phi_{22}^{w}-\varepsilon_{22}^{w}\right)}{\left(\phi_{22}^{h}-\varepsilon_{22}^{h}\right)-\left(\phi_{22}^{w}-\varepsilon_{22}^{w}\right)}$.

Although all the terms in the numerator are in $y^{2}$, since the model is constructed in such a way that $\left(\phi_{22}^{w}-\varepsilon_{22}^{w}\right)$ exactly satisfies the asymptotic behaviour of $\left(\phi_{22}^{*}-\varepsilon_{22}\right)$, their difference is in $y^{3}$, which suggests the use of either $f_{\phi}=f_{\varepsilon}=k \alpha$ or $f_{\phi}=f_{\varepsilon}=\alpha^{3}$, corresponding to $\lambda=k$ and $\lambda=\alpha^{2}$, respectively. As mentioned above, $f_{\phi}=k \alpha$ does not give fully satisfactory results, even in a simple channel flow, such that $f_{\phi}=\alpha^{3}$ is used in the most recent applications of the EB-RSM. Fig. 4a compares the a priori evaluation of $f_{\phi}$ using Eq. (47) with the model for $f_{\phi}$, using the three formulations $f_{\phi}=k \alpha, f_{\phi}=\alpha^{2}$ and $f_{\phi}=\alpha^{3}$. It can be seen that, as expected, the a priori evaluated $f_{\phi}$ goes to zero at the wall and to one far from the wall. Actually, it is observed that it does not exactly go to one, because the SSG model $\phi_{i j}^{h}$ and the Kolmogorov model $\varepsilon_{i j}^{h}=\frac{2}{3} \varepsilon \delta_{i j}$ do not perfectly reproduce the difference $\phi_{22}^{*}-\varepsilon_{22}$, but this feature is to be ignored, since the purpose of the Elliptic Blending method is to enforce the near-wall behaviour 
of this term, rather than correcting imperfections of the weakly inhomogeneous models far from the wall. The two formulations $f_{\phi}=\alpha^{2}$ and $f_{\phi}=\alpha^{3}$ are globally very similar and provide the correct blending between the near-wall and weakly inhomogeneous models, contrary to $f_{\phi}=k \alpha$ that does not properly go to one far from the wall, as pointed out above. It is to be noted that the different formulations require different calibrations of the coefficients $C_{L}$ involved in the model (33) for the length scale, as well as for the coefficient $A_{1}$ involved in the dissipation equation, as discussed in section 4.3. These two coefficients are calibrated in order to optimize the prediction of the evolution of the friction coefficient $C_{f}$ as a function of $R e_{\tau}$, as shown in Fig. 1b. Values are given in Appendix C.

Fig. 1b shows that the original version of the model, with $f_{\phi}=k \alpha$, leads to predictions significantly less satisfactory than the other two formulations. Fig. 5 compares the results obtained with $f_{\phi}=\alpha^{2}$ and $f_{\phi}=\alpha^{3}$ in channel flows from $R e_{\tau}=180$ up to $R e_{\tau}=4200$. Although the two formulations give very similar results, it can be seen in Fig. 5a that the mean velocity predictions in the buffer layer are improved using $f_{\phi}=\alpha^{3}$. Fig. 5b shows that the two formulations give comparable and satisfactory results for the $\overline{v^{2}}, \overline{w^{2}}$ and $\overline{u v}$ components of the Reynolds stress tensor. For the $\overline{u^{2}}$ component, significant differences are observed, in particular for the peak of $\overline{u^{2}}$ in the buffer layer. The formulation $f_{\phi}=\alpha^{2}$ very satisfactorily replicates the profile at low Reynolds number, but underestimates the peak value at high Reynolds number. On the contrary, the formulation $f_{\phi}=\alpha^{3}$ provides better predictions at high Reynolds number, such that, in order to favour accurate predictions for engineering applications, in which the Reynolds number is usually high, this formulation is preferred and used in the reference model. It is worth pointing out that in the log layer, where the SSG model $\phi_{i j}^{h}$ is active, the model is not able to reproduce the high-level plateau of $\overline{u^{2}}$. This feature is common to all the RANS models, and is to be traced to the presence in the DNS results of very large scale motions (Wu et al., 2012) coming from the central region of the channel, which the models are not able to account for.

Fig. 6 shows the profile of the blending function $f_{\phi}$, here using $f_{\phi}=\alpha^{3}$, for several values of the friction Reynolds number $R e_{\tau}$. Fig. $6 \mathrm{~b}$ shows that $f_{\phi}$ is independent of the Reynolds number when plotted as a function of $y^{+}$. This is the desired behaviour, since the distance over which the blockage effect of the wall remains influential scales with the integral length scale (Perot and Moin, 1995) that satisfies $L^{+}=\kappa y^{+}$in the wall layer. When $\alpha$ is plotted as a function of $y / h$ (Fig. 6a), it is consistently observed that the region of influence of the wall reduces when the Reynolds number increases.

\subsection{Dissipation equation}

The transport equation for the dissipation rate $\varepsilon$ used by Manceau and Hanjalić (2002) reads

$\frac{\mathrm{D} \varepsilon}{\mathrm{D} t}=\frac{C_{\varepsilon 1} P-C_{\varepsilon 2} \varepsilon}{T}+\frac{\partial}{\partial x_{l}}\left(\frac{C_{\mu}}{\sigma_{\varepsilon}} \overline{u_{l} u_{m}} T \frac{\partial \varepsilon}{\partial x_{m}}\right)+\nu \frac{\partial^{2} \varepsilon}{\partial x_{k} \partial x_{k}}+E$

where, following Durbin (1993), the time scale $T$ is the eddy-turnover time bounded by the Kolmogorov time scale, in order to avoid a singularity at the wall,

$T=\max \left(\frac{k}{\varepsilon}, C_{T}\left(\frac{\nu}{\varepsilon}\right)^{1 / 2}\right)$.

The last term of Eq. (48),

$E=C_{\varepsilon 3} \nu \frac{k}{\varepsilon} \overline{u_{j} u_{k}}\left(\frac{\partial^{2} U_{i}}{\partial x_{j} \partial x_{l}}\right)\left(\frac{\partial^{2} U_{i}}{\partial x_{k} \partial x_{l}}\right)$

is intended to represent the term in the exact $\varepsilon$-equation (Hanjalić and Launder, 2011),

$P_{\varepsilon 3}=-2 \nu \overline{u_{k} \frac{\partial u_{i}}{\partial x_{l}}} \frac{\partial^{2} U_{i}}{\partial x_{k} \partial x_{l}}$,

which peaks in the buffer layer, and is negligible in weakly inhomogeneous regions (Mansour et al., 1988), such that it is usually not accounted for in standard models. Anticipating potential numerical difficulties linked to the term $E$, Manceau and Hanjalić (2002) also proposed to mimic the effect of $P_{\varepsilon 3}$ by stimulating production of dissipation in the buffer layer through a variable $C_{\varepsilon 1}$, denoted by $C_{\varepsilon 1}^{\prime}$, inspired by Durbin and Laurence (1996), of the form

$C_{\varepsilon 1}^{\prime}=C_{\varepsilon 1}\left[1+A_{1}\left(1-f_{\phi}\right) \sqrt{\frac{k}{\overline{u_{i} u_{j}} n_{i} n_{j}}}\right]$

(for a historical perspective on the evolution of Elliptic Relaxation models, see Billard and Laurence, 2012). In Eq. (52), Manceau and Hanjalić (2002) introduced the factor $\left(1-f_{\phi}\right)$ in order to avoid any influence of this term far from the wall. It is to be noted that the term $\sqrt{k / \overline{u_{i} u_{j}} n_{i} n_{j}}$ is singular at the wall, since it reduces to $\sqrt{k / \overline{v^{2}}}$, where $\overline{v^{2}}$ is the component normal to the wall, which behaves in $y^{-1}$ in the vicinity of the wall. However, since it is in front of the production rate that goes to zero as $y^{3}$ (see Appendix A), the production of $\varepsilon$ remains negligible in this region compared to viscous diffusion and destruction of $\varepsilon$ in Eq. (48). Manceau (2003) adopted this modification and observed a significant improvement of the numerical robustness of the EB-RSM compared to the term $E$, and it was used in subsequent applications of the model. However, Dehoux et al. (2011) observed a misbehaviour of this term in natural convection and proposed

$C_{\varepsilon 1}^{\prime}=C_{\varepsilon 1}\left[1+A_{1}\left(1-f_{\phi}\right) \frac{P}{\varepsilon}\right]$.

A variable coefficient $C_{\varepsilon 1}^{\prime}$ function of the ratio $P / \varepsilon$ was already used by Durbin (1993) in order to reproduce the physical effect of the term $P_{\varepsilon 3}$, in the frame of the Elliptic Relaxation model, and by Waclawczyk et al. (2004), 

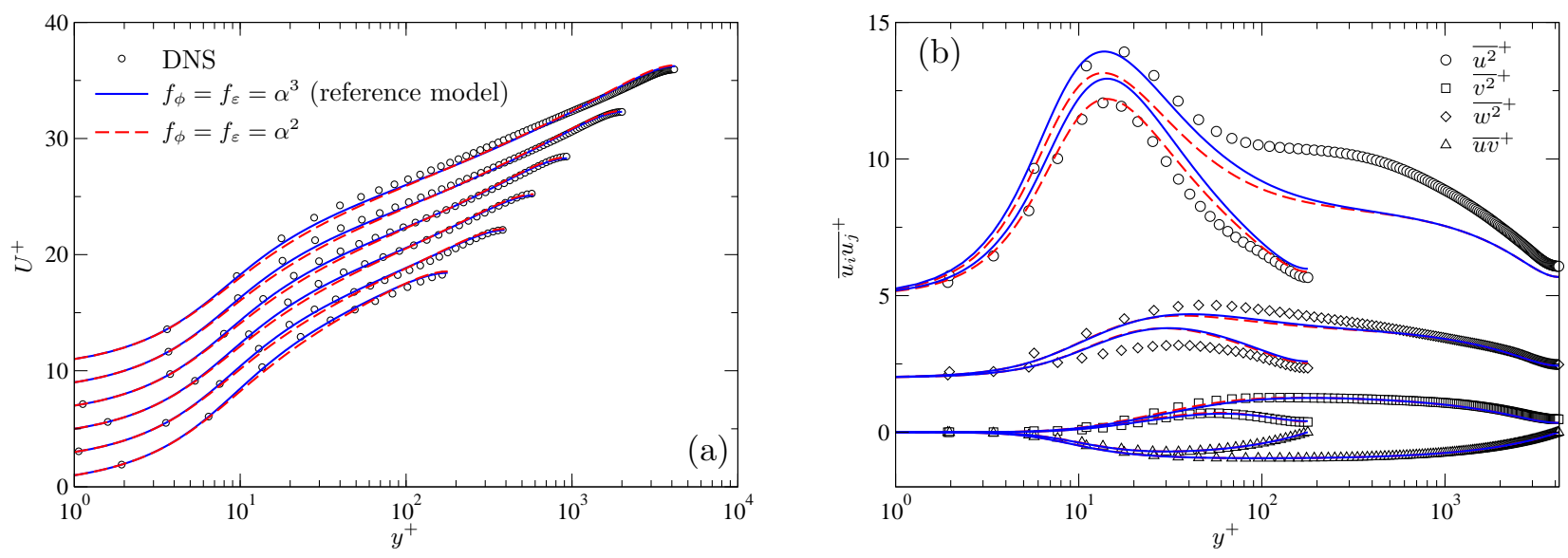

Figure 5: Comparison with DNS data (Moser et al., 1999; Lozano-Durán and Jiménez, 2014) of the results given in channel flows by two formulations of the EB-RSM. (a) Mean velocity profiles for $R e_{\tau}$ ranging from 180 to 4200 . (b) Reynolds stress profiles at $R e_{\tau}=180$ and 4200. $U, \overline{u^{2}}$ and $\overline{w^{2}}$ profiles are shifted for clarity.
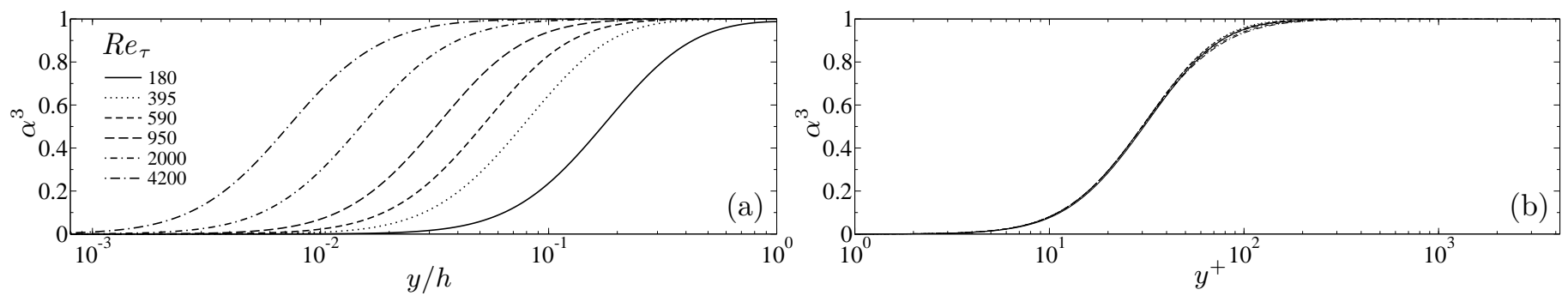

Figure 6: Evolution with $R e_{\tau}$ of the blending function $f_{\phi}=\alpha^{3}$ as a function of (a) $y / h$; (b) $y^{+}$.

in the frame of the EB-RSM. However, this model was later abandoned because the departure from unity of the ratio $P / \varepsilon$ in non-equilibrium flows can erroneously activate this term far from the wall (Durbin and Laurence, 1996). In Eq. (53), Dehoux et al. (2011) introduced the factor $\left(1-f_{\phi}\right)$ in order to restrict the effect of the term to near-wall regions. Recently, Manceau et al. (2014) experienced a lack of numerical robustness of Eq. (52) in the case of a jet impinging onto a rotating disk and adopted Eq. (53). This recent version is compared below with versions obtained by substituting other modelling options for the term $P_{\varepsilon 3}$. For the sake of completeness, the possibility of using $\left(1-f_{\phi}\right) E$ instead of $E$ is also investigated.

Fig. 1c shows a comparison, in the case of a channel flow, of the evolution of the friction coefficient with the Reynolds number. As explained above, the coefficient $C_{L}$ entering the length scale and the coefficient involved in the modelling of $P_{\varepsilon 3}$ (either $A_{1}$ or $C_{\varepsilon 3}$ depending on the formulation) are calibrated in order to optimize the prediction of $C_{f}$ in this figure. It is clearly seen that the particular model for $P_{\varepsilon 3}$ does not exhibit a significant influence on this curve. These results must not be misinterpreted: accounting for the physical role of $P_{\varepsilon 3}$ is absolutely necessary in order to obtain correct results; however, with a proper calibration of $C_{L}$ and either $A_{1}$ or $C_{\varepsilon 3}$, the different options are interchangeable.
Fig. 7 confirms this conclusion. All the results obtained for $U$ and the Reynolds stresses are superimposed, except at the lowest Reynolds number $R e_{\tau}=180$. For this case, a slightly better prediction of the mean velocity profile is observed with the terms $E$ and $\left(1-f_{\phi}\right) E$. However, as mentioned above, the EB-RSM is not intended to be a lowReynolds number model and does not include any specific modification. Owing to the significant gain in numerical robustness, the term given by Eq. (53) is then used in the reference model.

\section{Extension to rotating flows}

Rotating flows are of considerable importance to many industrial fields, in particular turbomachinery, or in geophysical and astrophysical applications. These flows are moreover very challenging for turbulence models (see, for instance, the review of Jakirlić et al., 2002). In particular, the case of a rotating channel with spanwise rotation is an idealization of the flow in turbine blade passages. The mean velocity equations being insensitive to rotation, since both the centrifugal and the Coriolis forces simply induce a modification of the mean pressure, the only influence of rotation on the mean velocity profile is through the $\overline{u v}$ component of the Reynolds stress (Launder et al., 1987). This test case is thus particularly relevant to the evaluation of the performance of a turbulence model. 

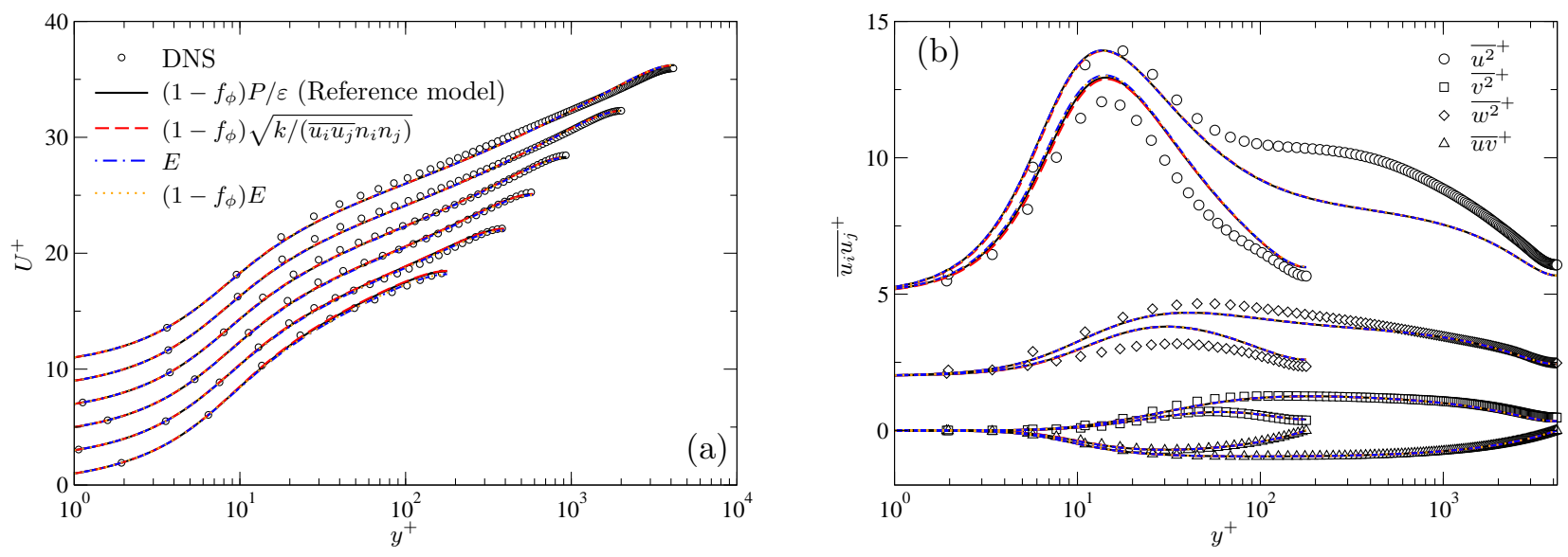

Figure 7: Same comparisons as in Fig. 5 for the different options for the modelling of the production term $P_{\varepsilon 3}$ in the exact transport equation for the dissipation rate $\varepsilon$.

Therefore, the case of the channel flow in spanwise rotation has been investigated by several authors, either experimentally (Johnston et al., 1972; Nakabayashi and Kitoh, 2005) or using DNS (Kristoffersen and Andersson, 1993; Lamballais et al., 1996, 1998; Nagano and Hattori, 2003; Grundestam et al., 2008). In order to assess the performance of the EB-RSM, the databases of Lamballais et al. (1998) and Grundestam et al. (2008) are used.

This type of flows has been widely investigated using RANS models, occasionally with Reynolds stress models (Launder et al., 1987; Belcher and Durbin, 1992; Wizman et al., 1996; Pettersson and Andersson, 1997; Iaccarino et al., 1999; Chaouat, 2001; Shin et al., 2003). Launder et al. (1987) first showed that, contrary to linear eddyviscosity models, Reynolds-stress models are by nature able to reproduce the influence of rotation in a turbulent channel flow, as soon as the Coriolis redistribution term ${ }^{1}$

$G_{i j}=-2 \omega_{k}\left(\epsilon_{i k m} \overline{u_{j} u_{m}}+\epsilon_{j k m} \overline{u_{i} u_{m}}\right)$

is introduced in the Reynolds-stress transport equation, and the model for the pressure-strain correlation is written in an objective manner. In order to satisfy the latter constraint, it can be shown that it is sufficient to write the model in terms of the mean rate of strain tensor $S_{i j}$ and the absolute mean vorticity tensor $W_{i j}$,

$W_{i j}=\frac{1}{2}\left(\frac{\partial U_{i}}{\partial x_{j}}-\frac{\partial U_{j}}{\partial x_{i}}\right)+\epsilon_{m j i} \omega_{m}$,

where $\boldsymbol{\omega}$ is the rotation axial vector. For details, see Speziale (1989) or the excellent article of Luca and Sadiki (2008).

Iaccarino et al. (1999) applied the SSG model to the case of a rotating channel flow, and obtained encouraging results. They nonetheless pointed out that the near-wall treatment they applied, a two-layer approach, is not satisfactory and is at the origin of the observed discrepancies. Belcher and Durbin (1992) and Wizman et al. (1996)

\footnotetext{
${ }^{1}$ Since the trace of this tensor is zero, the widely used denomination Coriolis production is considered misleading.
}

first showed that Durbin's Elliptic Relaxation model has the potential to reproduce the effect of the walls on turbulence in a rotating reference frame. Shin et al. (2003) tried to apply the original Elliptic Blending model to rotating channel flows. They faced numerical divergence for moderate rotation rates, and proposed a completely different model, albeit still based on the Elliptic Blending concept, and found necessary to introduce additional, nonlinear, terms. The robust version of the EB-RSM (Manceau, 2003), based on $f_{\phi}=\alpha^{2}$ and the variable coefficient $C_{\varepsilon 1}^{\prime}$ given by Eq. (52) (see sections 4.2 and 4.3, respectively), proved stable and gave globally satisfactory results in rotating channel flows. The purpose of the present section is to compare the various versions of the model in this configuration.

Since the Elliptic Blending model is based on nearwall asymptotic considerations, and, in a rotating reference frame, the budgets of the Reynolds stresses are modified by the presence of the Coriolis redistribution term (54), it is necessary to revisit the model. For a wall located at $y=0$, without any assumption on the rotation axial vector $\boldsymbol{\omega}$, the Coriolis term reads

$$
\begin{aligned}
G_{11} & =-4 \omega_{2} \overline{u w}+4 \omega_{3} \overline{u v} ; \\
G_{22} & =4 \omega_{1} \overline{v w}-4 \omega_{3} \overline{u v} ; \\
G_{33} & =-4 \omega_{1} \overline{v w}+4 \omega_{2} \overline{u w} ; \\
G_{12} & =2 \omega_{1} \overline{u w}-2 \omega_{2} \overline{v w}+2 \omega_{3} \overline{v^{2}}-2 \omega_{3} \overline{u^{2}} ; \\
G_{13} & =-2 \omega_{1} \overline{u v}+2 \omega_{3} \overline{v w}-2 \omega_{2} \overline{w^{2}}+2 \omega_{2} \overline{u^{2}} \\
G_{23} & =2 \omega_{2} \overline{u v}-2 \omega_{3} \overline{u w}+2 \omega_{1} \overline{w^{2}}-2 \omega_{1} \overline{v^{2}} .
\end{aligned}
$$

Using the asymptotic behaviour in the vicinity of the wall of the fluctuating velocities $u=\mathcal{O}(y), v=\mathcal{O}\left(y^{2}\right)$ and $w=\mathcal{O}(y)$, it is straightforward to show that

$$
\begin{gathered}
G_{11}=\mathcal{O}\left(y^{2}\right) ; G_{22}=\mathcal{O}\left(y^{3}\right) ; G_{33}=\mathcal{O}\left(y^{2}\right) ; \\
G_{12}=\mathcal{O}\left(y^{2}\right) ; G_{13}=\mathcal{O}\left(y^{2}\right) ; G_{23}=\mathcal{O}\left(y^{2}\right) .
\end{gathered}
$$

Comparing to the behaviour of other terms of the budget (see Appendix A), it can be seen that $G_{i j}$ is negligible 
in the vicinity of the wall for all the components of the Reynolds stress, since, in particular,

$$
\frac{G_{i j}}{\phi_{i j}^{*}}=\mathcal{O}\left(y^{-1}\right)
$$

Therefore, it is not necessary to account for the presence of the Coriolis redistribution term in the near-wall part $\phi_{i j}^{w}$ of the model: the EB-RSM does not require any other modification than Eqs. (54) and (55) for rotating flows (possible modifications of the dissipation equation are discussed below).

The model is evaluated against the DNS data, at $R e_{b}=$ $U_{b} h / \nu=2500$, where $U_{b}$ is the bulk velocity and $h$ the halfwidth of the channel, and the highly-resolved LES data, at $R e_{b}=7000$, of Lamballais et al. (1998), which are available for 4 different rotation numbers $R o=2 \omega h / U_{b}=0,1 / 6,0.5,1.5$; complemented by DNS data of Grundestam et al. (2008) at $R e_{\tau}=180$ and higher rotation numbers, ranging from 0.98 to 3.0 , up to complete laminarization (see table 2).

Computations are performed using the same 1D code as used in section 4. Grid convergence was carefully enforced, with up to 400 grid points in the channel, although it was found that 100 points were sufficient even at $R e_{b}=7000$. For each formulation of the model and each value of the Reynolds number, 133 computations were automatically run in order to span the range of rotation numbers from 0 to 4 , such that the results presented in Fig. 8 consist of about 1200 computations.

The global behaviour of the flow for increasing rotation numbers is well summarized by this figure, which shows the modification of the friction velocity $u_{\tau}=\sqrt{\tau_{w} / \rho}$ on both sides of the channel compared to the case without rotation, for the two values of the Reynolds number, $R e_{b}=2500$ and 7000, corresponding to the DNS and LES data of Lamballais et al. (1998), respectively. Although computations were performed with all the versions of the EB-RSM mentioned in section 4 , only a representative selection is shown in the figure, for the sake of conciseness. The legend describes the distinct versions, mainly by indicating the formulation used for the elliptic blending function $f_{\phi}\left(\alpha^{3}, \alpha^{2}\right.$ or $k \alpha)$ and the model for $P_{\varepsilon 3}\left(\left(1-f_{\phi}\right) P / \varepsilon,\left(1-f_{\phi}\right) k / \sqrt{\overline{v^{2}}}\right.$, $\left(1-f_{\phi}\right) E$ or $\left.E\right)$. Three additional curves are plotted in order to illustrate particular aspects, as described below (low-Re effect, influence of the nonlinear term in the SSG model, modification of the energy cascade).

Firstly, in order to illustrate the reasons why the original version of the model $(k \alpha, E)$ was discarded in section 4.2 , it is compared to LES data at high Re in Fig. 8b. Although the results obtained for low rotation rates appear very satisfactory in this figure, the friction velocity on both sides do not show the correct trend after $R o=1 / 6$ and the computation diverges for $R o>0.3$. As seen in Fig. $4 \mathrm{~b}$, the model gives erroneous profiles on the cyclonic side, mainly due to a misbehaviour of the blending function $f_{\phi}=k \alpha$ in case of laminarization, such that a blending function independent of $k$ is preferred.
Fig. 8 shows that other versions of the model yield results with modest differences. In particular, the version of Manceau (2003) $\left(\alpha^{2} ;\left(1-f_{\phi}\right) k / \sqrt{\overline{v^{2}}}\right)$, and the present version (reference model, $\alpha^{3}$ and $\left(1-f_{\phi}\right) P / \varepsilon$ ) are very close to each other. This result shows that, contrary to the case of the blending function mentioned just above, the presence of $k$ in the model for $P_{\varepsilon 3}$ is not problematic in case of laminarization. It is also worth pointing out that the inclusion of the nonlinear term (i.e., the second term in the right hand side of Eq. (34), with $g_{2}=4.2$ ) does not significantly affect the results (Fig. 8b). The version using $\left(1-f_{\phi}\right) E$ (or, equivalently, $E$ ) instead of $\left(1-f_{\phi}\right) P / \varepsilon$ gives slightly different results, and shows better or worse predictions, depending on the side of the channel and the rotation number. Since none of the alternative versions shows decisive advantages, in the remainder of the present article, only the results using the reference model will be shown.

It can be seen that, on the cyclonic (or suction) side, for weak values of $R o$, the friction velocity decreases almost linearly with $R o$, a result supported by experimental studies (e.g., Johnston et al., 1972). The trend suddenly changes for a value of $R o$ that depends on the Reynolds number, and the friction reaches a plateau or slightly increases, and eventually, a final decrease towards complete laminarization is observed. Although the number of rotation rates for which DNS/LES data are available is not sufficient to confirm this shape of the curve, the results given by the EB-RSM appear consistent with the data. On the anticyclonic side, a sharp increase of the friction velocity is initially obtained. The maximum is reached at about $R_{o}=0.45$ and $R o=0.25$ for the lower and higher values of the Reynolds number, respectively. For higher rotation rates, a laminarization is gradually observed on this side of the channel as well, and complete relaminarization is obtained for $R_{0}>3.5$, for which a symmetric, laminar friction velocity is reached.

Comparing Figs. 8a and $8 \mathrm{~b}$ shows that the results at the lower Reynolds number are less satisfactory than those at the higher Reynolds number. This is to be related to the fact that the model is purposely calibrated to reproduce high Reynolds number flows, as mentioned in section 4. Fig. 8a shows that, when the model is artificially calibrated in order to optimize the prediction of the nonrotating channel flow at $R e_{\tau}=180$ (using $C_{L}=0.105$ ) the reproduction of the evolution of the friction velocity with the rotation number is significantly improved for the lower bulk Reynolds number. Consequently, the relative misprediction of this evolution using the reference model is considered a low-Reynolds number effect and of minor importance for industrial applications.

In contrast, for the higher value of the Reynolds number, Fig. 8b, the predictions are globally satisfactory. However, it is observed that the model is slightly over-sensitive to rotation for weak rotation numbers, on both sides of the channel. Moreover, on the anticyclonic side, after the 
Table 2: List of DNS (top) and LES (bottom) databases used for comparison. Bold-faced cells correspond to data used in Fig. 9.

\begin{tabular}{|c|c|c|c|c|}
\hline Source & \multicolumn{4}{|c|}{ Lamballais et al. (1998) } \\
\hline Ro & 0.00 & $1 / 6$ & 0.50 & 1.50 \\
\hline$R e_{\tau}$ & 162 & 162 & 151 & 104 \\
\hline$R e_{b}$ & 2500 & 2500 & 2500 & 2500 \\
\hline Source & \multicolumn{4}{|c|}{ Lamballais et al. (1998) } \\
\hline Ro & 0.00 & $1 / 6$ & 0.50 & 1.50 \\
\hline$R e_{\tau}$ & 386 & 361 & 317 & 223 \\
\hline$R e_{b}$ & 7000 & 7000 & 7000 & 7000 \\
\hline
\end{tabular}
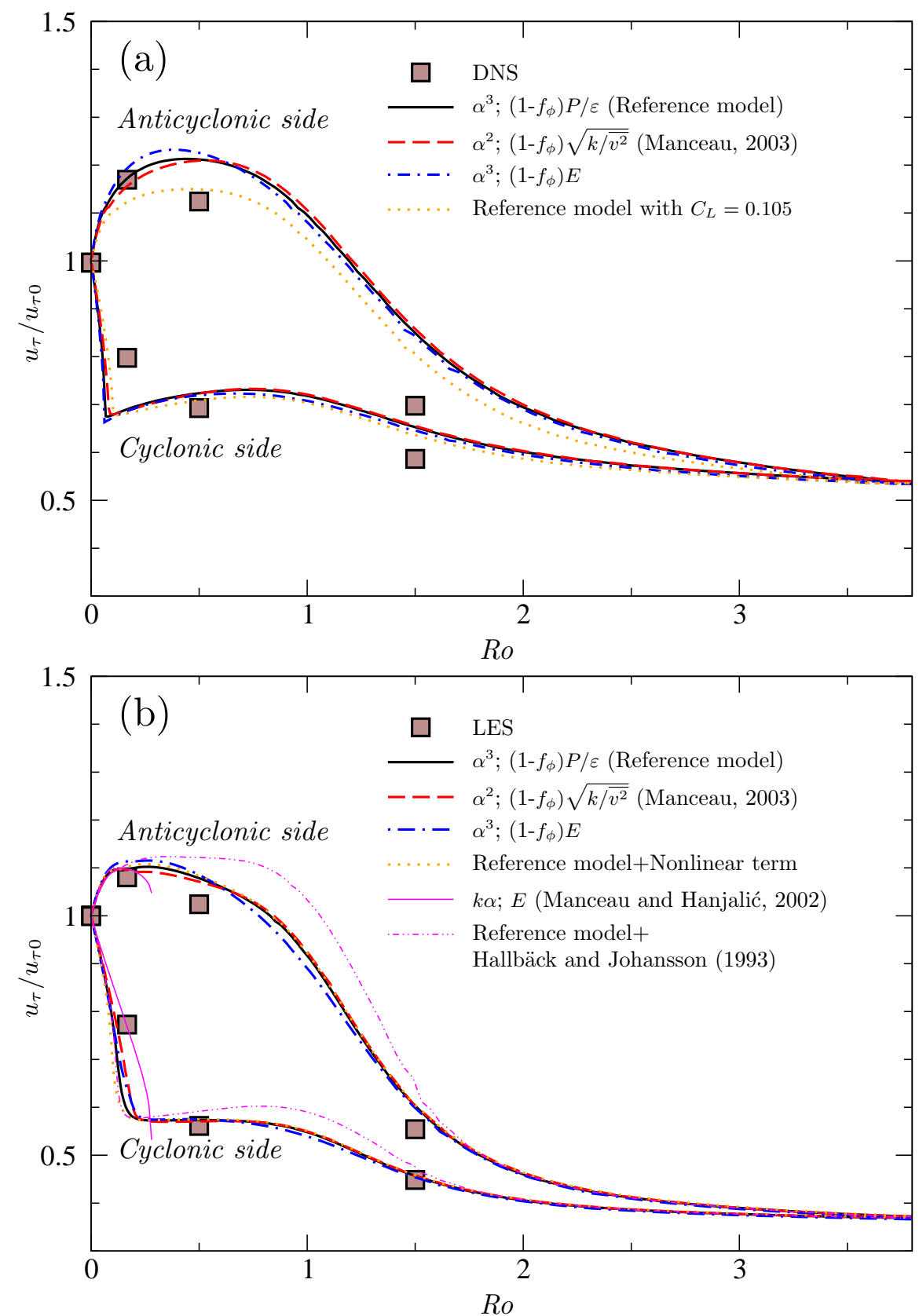

Figure 8: Rotating channel flows at: (a) $R e_{b}=2500$ (b) $R e_{b}=7000$. Investigation of the influence of the rotation number on the wall friction. Ratio of the friction velocity $u_{\tau}$ on both sides of the channel to the friction velocity for the non-rotating case $u_{\tau 0}$. Comparison with the DNS and LES data of Lamballais et al. (1998). 
maximal friction velocity is reached, the laminarization is too slow. In order to investigate possible improvement of these predictions, modifications of the dissipation equation are tested. Indeed, Bardina et al. (1983) emphasized the necessity of making the $\varepsilon$-equation sensitive to rotation, in order to account for the retardation of the energy cascade (Jacquin et al., 1990; Sagaut and Cambon, 2008) and many modifications were proposed in the literature (for a recent review, see Jakirlić et al., 2002). Here, four modifications, proposed by Bardina et al. (1985), Hallbäck and Johansson (1993), Shimomura (1993) and Rubinstein and Zhou (1997), respectively, were introduced in order to investigate their influence of the prediction of the friction velocities shown in Fig. 8. All these modifications aim at reducing the dissipation rate in rotating cases by increasing the destruction term in its transport equation, and yield similar incorrect results in the present case. To illustrate the effect of such terms, the results obtained with the modification of Hallbäck and Johansson (1993), which consists in sensitizing the destruction term of $\varepsilon$ to the rotation rate by multiplying the $C_{\varepsilon 2}$ coefficient by the factor

$1+\frac{A \sqrt{R e_{t}}}{C_{\varepsilon 2}\left(25+2 \omega^{*}\right)} \omega^{*}$

where $R e_{t}$ is the turbulent Reynolds number $R e_{t}=k^{2} /(\nu \varepsilon)$ and $\omega^{*}=\omega k / \varepsilon$, are shown in Fig. $8 \mathrm{~b}$. It can be seen that this modification has a dramatic effect on the results: the recommended coefficient $A=0.6$ had to be reduced here to 0.1 to avoid a rapid departure from a physically admissible behaviour. Moreover, the introduction of this term does not improve the results: for small values of $R o$, the term has the undesired effect of increasing the sensitivity of the model to rotation; for large values of $R o$, the laminarization is delayed, on both sides of the channel. Such modifications are intended to reproduce the effect of the inhibition of the energy cascade in homogeneous turbulence, and the present results suggest that they are not adequate for improving the results of the EB-RSM in wall-bounded, rotating, flows. Moderating the sensitivity of the model to rotation at low rotation rates and accelerating the laminarization at high rotation rates thus remains an open issue.

Since the data of Lamballais et al. (1998) are limited to $R o \leq 1.5$, in order to evaluate the model for very high rotation numbers, up to complete laminarization, computations are carried out using flow parameters corresponding to the data of Grundestam et al. (2008) (see table 2). For the purpose of spanning the whole range of rotation numbers from 0 to 3, a composite figure is plotted (Fig. 9), using all the available data corresponding to values of $R e_{\tau}$ close to 180. The list of DNS and LES computations used in this figure is given in table 2 (bold-faced cells). Here, since neither the bulk Reynolds number nor the friction Reynolds number are constant, the friction velocity is not made non-dimensional using the value for the corresponding non-rotating case, but rather the average friction velocity defined by $u_{\tau a}^{2}=\left(u_{\tau 1}^{2}+u_{\tau 2}^{2}\right) / 2$, where $u_{\tau 1}$ and $u_{\tau 2}$

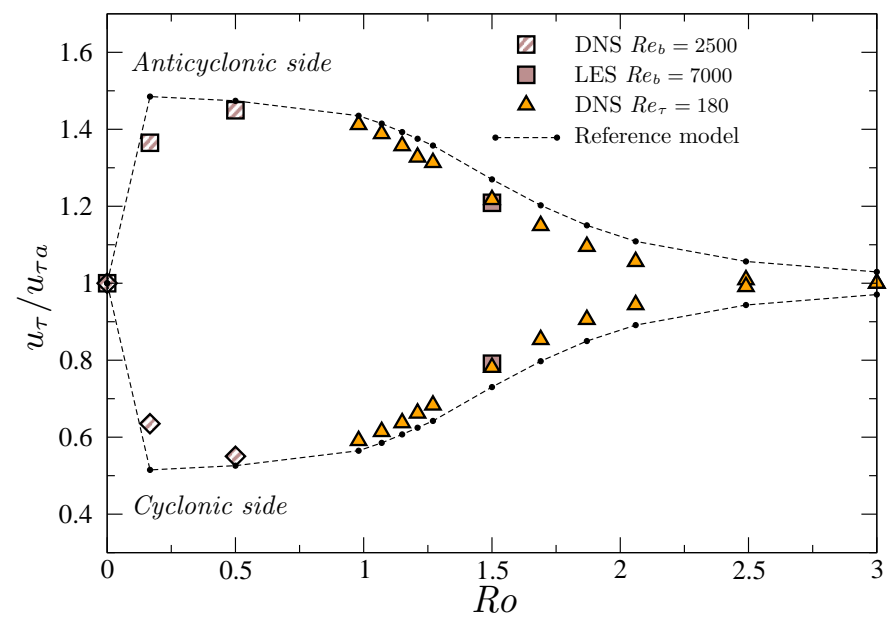

Figure 9: Rotating channel flow at $R e_{\tau} \simeq 180$. Ratio of the friction velocity $u_{\tau}$ on both sides of the channel to the average friction velocity $u_{\tau a}$. Comparison with the DNS and LES data of Lamballais et al. (1998) and the DNS data of Grundestam et al. (2008).

are the friction velocity evaluated at the two walls. For $R o \leq 1.5$, Fig. 9 obviously confirms what was observed in Fig. 8, i.e., a slight over-sensitivity of the model to the effects of rotation. For high rotation numbers, it is observed that the model predicts the laminarization in the whole domain in a proper way, with an excellent reproduction of the shape of the curves, although it is slightly delayed compared to the DNS: Grundestam et al. (2008) observed complete laminarization $\left(u_{\tau 1}=u_{\tau 2}\right)$ for $R o \simeq 3$, while the model maintains a weak level of turbulence on the anticyclonic side up to $R o \simeq 3.5$.

Fig. 10 shows the influence of the rotation rate on the mean velocity, kinetic energy and shear stress profiles, for low (top) and high (bottom) Reynolds numbers. When the rotation rate is increased, the velocity profile becomes asymmetric, in response to the asymmetry of the shear stress. Three regions can be identified: the two boundary layers, on the cyclonic (suction) and anticyclonic (pressure) sides, separated by the core region, characterized by a zero absolute mean vorticity (see Eq. 55) (Johnston et al., 1972; Kristoffersen and Andersson, 1993; Oberlack, 2001), such that, using the half-width of the channel $h$ and the bulk velocity $U_{b}$ as reference units, we have

$\frac{\partial U / U_{b}}{\partial y / h}=R o$

in this region. In the framework of homogeneous shear flows, it can be shown (Bradshaw, 1969; Johnston et al., 1972; Bertoglio, 1982; Speziale and Mac Giolla Mhuiris, 1989 ) that the effect of rotation on the turbulent field can be either stabilizing for $B>0$ or destabilizing for $B<0$, where $B$ is the Bradshaw number defined as

$B=-\frac{2 \omega}{S}\left(1-\frac{2 \omega}{S}\right)$

where $S=\partial U / \partial y$. Here, $\omega>0$, such that, on the cyclonic side, where $S<0$, rotation always has a stabilizing effect. 

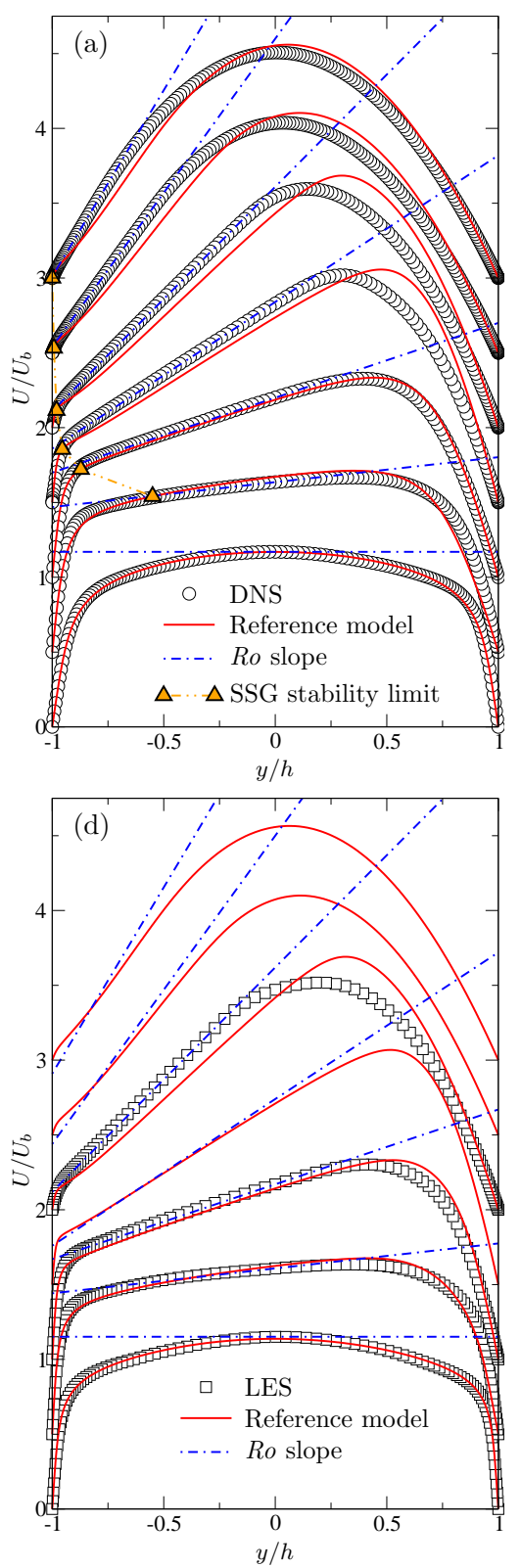

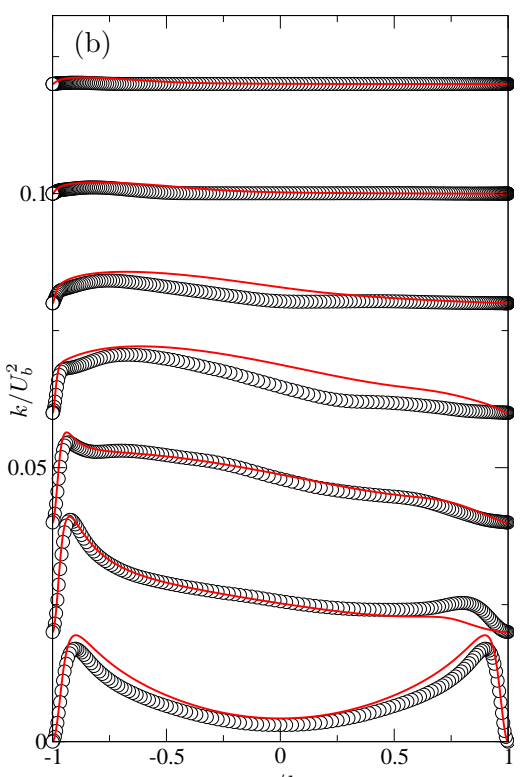

$y / h$

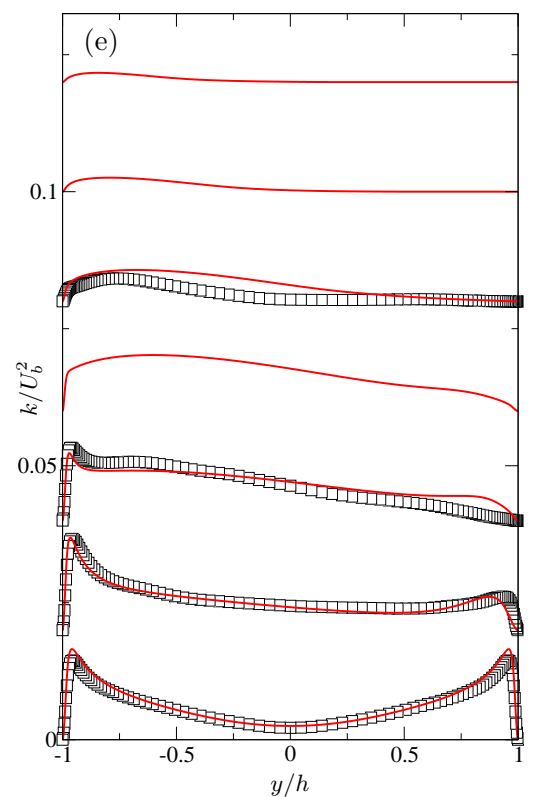

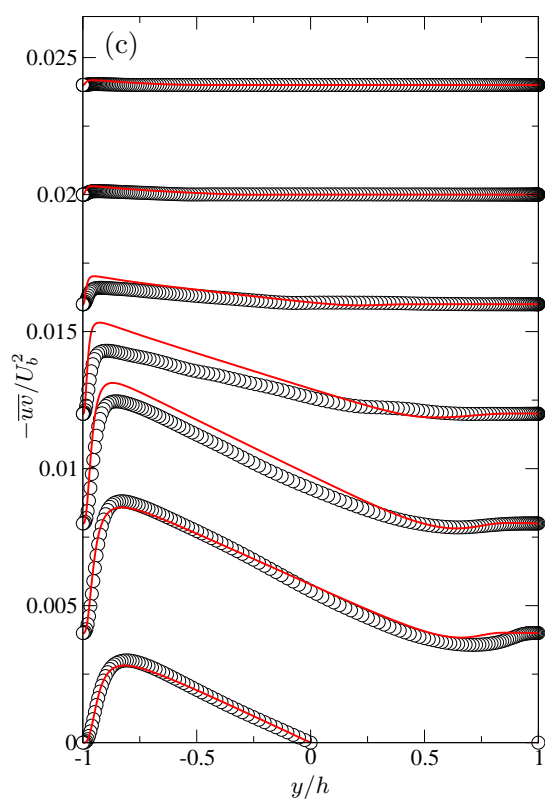

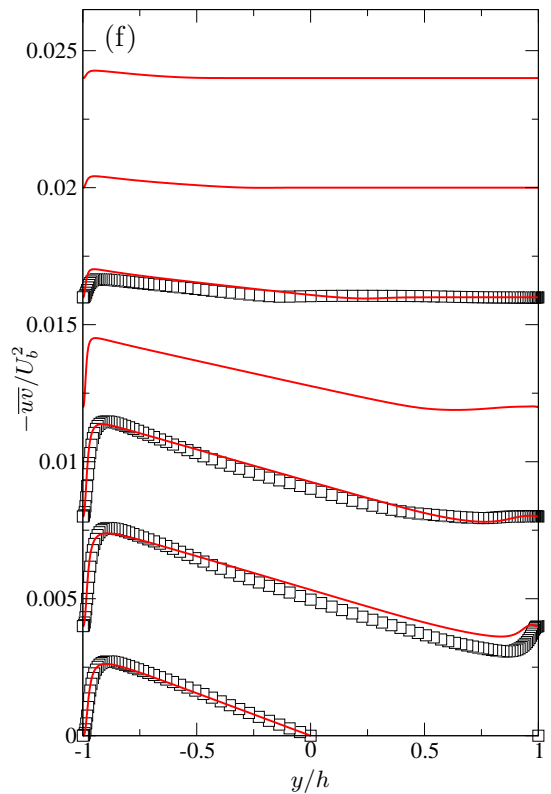

Figure 10: Rotating channel flow. Evolution with the rotation number of $(\mathrm{a}, \mathrm{d})$ the mean velocity profile; (b,e) the turbulent energy profile and $(\mathrm{c}, \mathrm{f})$ the shear stress profile. (a,b,c) Low Reynolds number: comparisons with the DNS data of Lamballais et al. (1998) at $R e_{b}=2500$ for $R o=0,1 / 6$ and 0.5 and with the DNS data of Grundestam et al. (2008) at $R e_{\tau}=180$ for $R o=0.98,1.50,2.06$ and 2.49. (e,f,g) High Reynolds number: comparisons with the LES data of Lamballais et al. (1998) at $R e_{b}=7000$ for $R o=0,1 / 6,0.5$ and 1.5 . Profiles are shifted for clarity.

On the contrary, rotation has a destabilizing effect on the anticyclonic side $(S>0)$ for moderate rotation rates, and a stabilizing effect for strong rotation rates $(\omega>S / 2)$. The core region, where the velocity profile is nearly linear, according to Eq. (60), is a region of neutral stability. It can be seen in Figs. 10a and d that the model is able to reproduce these effects, mainly because a particular attention was paid by Speziale et al. (1991) in the derivation of the SSG model to the reproduction of the stability diagram of rotating, homogeneous shear flows. In particular, for such a flow, an unstable equilibrium solution is obtained for $-0.09<\omega / S<0.53$, which is close to the theoretical range $0<\omega / S<0.5$ corresponding to $B<0$. Therefore, on the anticyclonic side, as long as the mean shear $S$ is larger than the threshold $\omega / 0.53$, the flow is locally unstable. In Fig. 10a, the point of the velocity profile where this threshold is reached is highlighted, indicating that the flow is unstable in the region between the anticyclonic wall and this particular location. It is observed that the extent of this region very rapidly reduces with increasing rotation rates. Although the theoretical unstable range $0<\omega / S<0.5$ is valid for homogeneous flows only, it can be expected, as emphasized by Grundestam et al. (2008), that turbulence cannot be sustained if the threshold $\omega / 0.5$ is larger than 
the peak value of $S$ for the laminar velocity profile, i.e., the value at the anticyclonic wall. In units based on $h$ and $U_{b}$, this maximal value of $\tilde{S}=h S / U_{b}$ is 3 , and the Bradshaw number writes

$$
B=-\frac{R o}{\tilde{S}}\left(1-\frac{R o}{\tilde{S}}\right),
$$

such that the criterion of Grundestam et al. (2008) for a sustainable turbulent activity is $R o<3$. It is indeed observed in Fig. 9 that a fully laminar solution is obtained by DNS at $R o=3$. Since the instability range of the SSG model extents to 0.53 instead of 0.5 , a residual turbulent energy is obtained beyond this value, up to $R o=3.5$ (see Fig. 8), which is slightly above the theoretical threshold for the SSG model $R o=3 \times 0.53 / 0.5=3.18$ (it is worth recalling that this threshold strictly holds for homogeneous turbulence only). This is the main reason for the observed delay in the laminarization of the flow with the EB-RSM for high rotation rates. Moreover, the reason for the underestimation of the slope of the mean velocity profile in the core region by the EB-RSM is also linked to the slight overestimation of the bifurcation threshold by the SSG model. Indeed, this region is a region of neutral stability, such that the slope (60), plotted in Figs. 8a and d, corresponds $\omega / S=0.5$. The EB-RSM underestimates the slope by a factor $0.53 / 0.5$.

As emphasized by Iaccarino et al. (1999), the near-wall treatment associated with the model can be detrimental to the reproduction of such subtle physical mechanisms and the Elliptic Blending treatment is, in this respect, satisfactory. However, it is observed on the cyclonic side that the mean velocity gradient is slightly too low for the weakest rotation rate $(R o=1 / 6)$, in agreement with the too rapid laminarization observed in Fig. 8. For higher rotation rates $(R o \geq 0.98)$, the core region extends too far towards the cyclonic wall, a discrepancy linked to the delayed laminarization on this side of the channel, at the origin of a slow convergence of the velocity profile towards a parabola. This feature leads to a local overestimation of the mean velocity, and, in order to satisfy the mass flow rate, an underestimation in the rest of the channel. Therefore, it should be pointed out that the most visible discrepancy in the results, i.e., the underestimation of the mean velocity in the core region at high rotation rates, is an indirect consequence of the delayed laminarization on the cyclonic side when the rotation number is gradually increased, linked to the overestimated bifurcation threshold $\omega / S=0.53$ of the SSG model.

It is consistently observed in figure Fig. 10b,e and c,f that the damping of the turbulent kinetic energy and the shear stress on the cyclonic side is overestimated for low rotation rates and underestimated for high rotation rates. On the anticyclonic side, for weak rotations, the correct intensification of turbulence is obtained. The stabilization for higher rotation rates is correctly reproduced as well, although, for the low Reynolds number (Fig. 10c), the shear stress is overestimated for $0.5<R o<1.5$. This obser- vation is consistent with Fig. 8a that shows a significant delay of the laminarization on the anticyclonic side for this Reynolds number.

Fig. 11 shows the turbulent anisotropy $b_{i j}=\overline{u_{i} u_{j}} /(2 k)-$ $1 / 3 \delta_{i j}$ for the non-rotating case and the case $R o=0.98$. The influence of rotation on the anisotropy is strong and very different depending on the location. The DNS data exhibit a separation of the domain into the three regions already identified in Fig. 10a and d: the anticyclonic boundary layer, the core region and the cyclonic boundary layer, in the range $-1<y / h<-0.9,-0.9<y / h<0.25$ and $0.25<y / h<1$ in Fig. 11b, respectively. In addition, very close to the two walls, sublayers can be identified, characterized by stronger fluctuations in the spanwise direction than in the streamwise direction $\left(b_{33}>b_{11}\right)$. The EB-RSM is able to reproduce all these regions except the sublayer nearby the anticyclonic wall, but their extent is not perfectly reproduced: in particular, the excessive extension of the core region towards the cyclonic wall, already mentioned above, is observed in the figure. In the anticyclonic and cyclonic boundary layers, the anisotropy is similar to that of the non-rotating case, with $b_{11}>b_{33}>b_{22}$, except in the two sublayers, where $b_{33}$ exceeds $b_{11}$. These regions are in particular constrained by the blockage effect, that imposes $b_{22}=-1 / 3$ at the walls (two-component limit), which is exactly fulfilled by the EB-RSM. In contrast, the core region is characterized by a complete inversion of the components of the anisotropy, with $b_{22}>b_{33}>b_{11}$, due to the the Coriolis redistribution that takes energy to the streamwise Reynolds stress component $\overline{u^{2}}$ and redistributes it to the wall normal component $\overline{v^{2}}$.

Fig. 12 shows the budgets of the Reynolds stresses for the same two cases, in wall units and in semi-log scale, for the sake of clarity. Due to the appearance of the core region, the terms of the budget do not go to zero in the center of the channel. In the cyclonic boundary layer $\left(y^{+}>250\right)$, all the terms are strongly damped. Another striking feature is that the terms of the budgets of the components $\overline{v^{2}}$, $\overline{w^{2}}$ and $\overline{u v}$ are very significantly amplified compared to the non-rotating case: maximal values are increased by a factor about ten. This is clearly due to the presence of Coriolis redistribution, which is much more efficient than pressure redistribution ${ }^{2}$. This mechanism can be highlighted by focusing on the components $\overline{u^{2}}$ and $\overline{v^{2}}$. In the nonrotating case, turbulence extracts energy from the mean flow through the $P_{11}$ component only. In the budget of $\overline{u^{2}}$, this term is partly compensated by a relatively modest redistribution of energy to other components, for which it is the only source of energy. In the rotating case, this picture is completely modified, in particular in the core region, beyond $y^{+}=20$ : Coriolis production redistributes to $\overline{v^{2}}$ all the energy transferred from the mean flow to turbulence

\footnotetext{
${ }^{2}$ Although, for reasons explained above, the velocity-pressure gradient term is not decomposed into pressure diffusion and redistribution, the main effect of this term, in particular far from the walls, is a redistribution of energy among the normal stresses.
} 


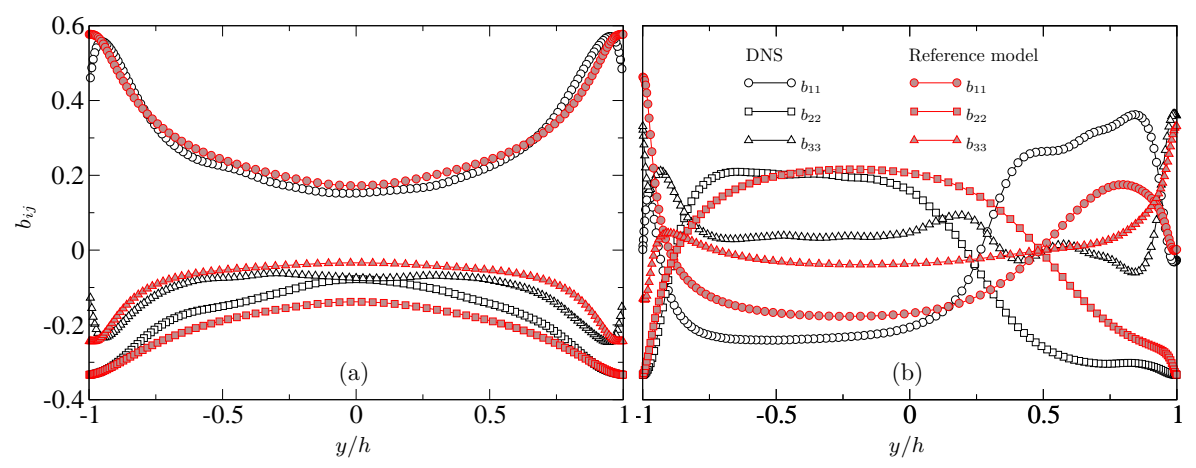

Figure 11: Rotating channel flow. Profiles of the normal components of the turbulent anisotropy for (a) $R o=0\left(R_{b}=2500\right) ;(\mathrm{b}) R o=0.98$ $\left(\operatorname{Re}_{\tau}=180\right)$.

$\left(G_{11}=-G_{22} \simeq-P_{11}\right)$. This is to be traced to the linear velocity profile observed in this region, with the slope $2 \omega$, which is equivalent to

$P_{11}=-2 \overline{u v} \frac{\partial U}{\partial y}=-4 \omega \overline{u v}=-G_{11}$.

This mechanism explains the high level of fluctuations in the wall-normal direction observed in Fig. 11. In comparison with the non-rotating case, the main source term in the $\overline{v^{2}}$-equation is 8 times larger, of the same order of magnitude as production. The velocity-pressure gradient term, which usually redistributes energy from the rich component $\overline{u^{2}}$ to the poor components $\overline{v^{2}}$ and $\overline{w^{2}}$ (non-rotating case), then decides to change its sign and to take energy from the nouveau riche $\overline{v^{2}}$ in favour of $\overline{w^{2}}$.

Although the distinction between rapid and slow parts of redistribution is not made in the DNS database, the following can be conjectured: these two components are in competition in the budget of $\overline{u^{2}}$. Indeed, as can be seen in Fig. $11 \mathrm{~b}, \overline{u^{2}}$ is very small in the core region. Actually, since $P_{11}+G_{11} \simeq 0$, it is only supplied with energy by turbulent diffusion (Fig. 12e). Therefore, it is expected that the slow part redistributes energy to $\overline{u^{2}}$, in order to resist strong anisotropies (Weinstock and Burk, 1985). In contrast, the rapid part is usually interpreted as an isotropization of the production processes (Naot et al., 1973), but, for reasons put forth by Launder et al. (1987), the redistributed source term is $P_{i j}+\frac{1}{2} G_{i j}$ rather than $P_{i j}+G_{i j}$, such that the rapid part is expected to take energy from $\overline{u^{2}}$, even though this component is weak. Fig. 12e shows that, in total, the slow and rapid terms nearly compensate in the core region. The decrease of the redistribution from $\overline{u^{2}}$ to other components is reproduced by the model, with a change of sign of the slow part compared to the non-rotating case (not shown here). However, the balance between the slow and rapid parts is not exactly obtained, and the velocity-pressure gradient term is slightly positive.

The term $\phi_{33}^{*}$ remains the only source of energy for $\overline{w^{2}}$ (Fig. 12g), but, contrary to the non-rotating case, energy is mainly redistributed from $\overline{v^{2}}$ rather than $\overline{u^{2}}$. In the region $y^{+}<10$, the model severely underestimates $\phi_{33}^{*}$, which, together with the prediction of a wrong sign of $\phi_{11}^{*}$, explains why the inversion of $b_{11}$ and $b_{33}$ in this region is not reproduced.

Fig. $12 \mathrm{~h}$ shows that for $-\overline{u v}$, the Coriolis term has a completely different effect in the anticyclonic boundary layer and the core region, with a change of sign located at $y^{+} \simeq 20$. In the anticyclonic boundary layer, it is a source term (i.e., it is positive in the budget of $-\overline{u v}$ ), and over-weights the production by the mean shear. The sum of this two terms is mainly balanced by $\phi_{12}^{*}$. The amplitude of this term is quite well predicted by the model in this region, which again validates the choice, made in section 4.1 , for the near-wall model $\phi_{12}^{w}$. The change of sign of $G_{12}$ is linked to the fact that wall-normal fluctuations become more energetic than streamwise fluctuations (see Fig. 11b), since we have

$G_{12}=-2 \omega\left(\overline{u^{2}}-\overline{v^{2}}\right)$.

In the core region, since $\overline{v^{2}}$ is much larger than $\overline{u^{2}}, G_{12}$ becomes a strong sink term in the budget of $-\overline{u v}$. In contrast with the case of the $\overline{u^{2}}$-equation, this term and production do not balance each other, since their sum

$$
\begin{aligned}
P_{12}+G_{12} & =-\overline{v^{2}} S-2 \omega\left(\overline{u^{2}}-\overline{v^{2}}\right) \\
& =-2 \overline{v^{2}} \omega-2 \omega\left(\overline{u^{2}}-\overline{v^{2}}\right) \\
& =-2 \omega \overline{u^{2}}
\end{aligned}
$$

remains a source term for $-\overline{u v}$. This source term is balanced by $\phi_{12}^{*}$, which is correctly reproduced by the model.

In conclusion, the analysis of Figs. 11 and 12 shows that the main mechanisms underlying the strong modification of the structure of turbulence in rotating channel flows are correctly reproduced by the EB-RSM, although the amplitudes of the different terms are not always correct and the extents of the different regions, characterized by different dominant mechanisms, are not perfectly predicted. Many of these good properties are to be attributed to the SSG model, but the use of the Elliptic Blending strategy to extend the validity of this model to the near-wall region appears relevant, and, in view of the satisfactory reproduction of the effects of rotation on the mean velocity profiles and the Reynolds stresses, it is felt that the version of the EB-RSM selected above, called herein the reference model, 

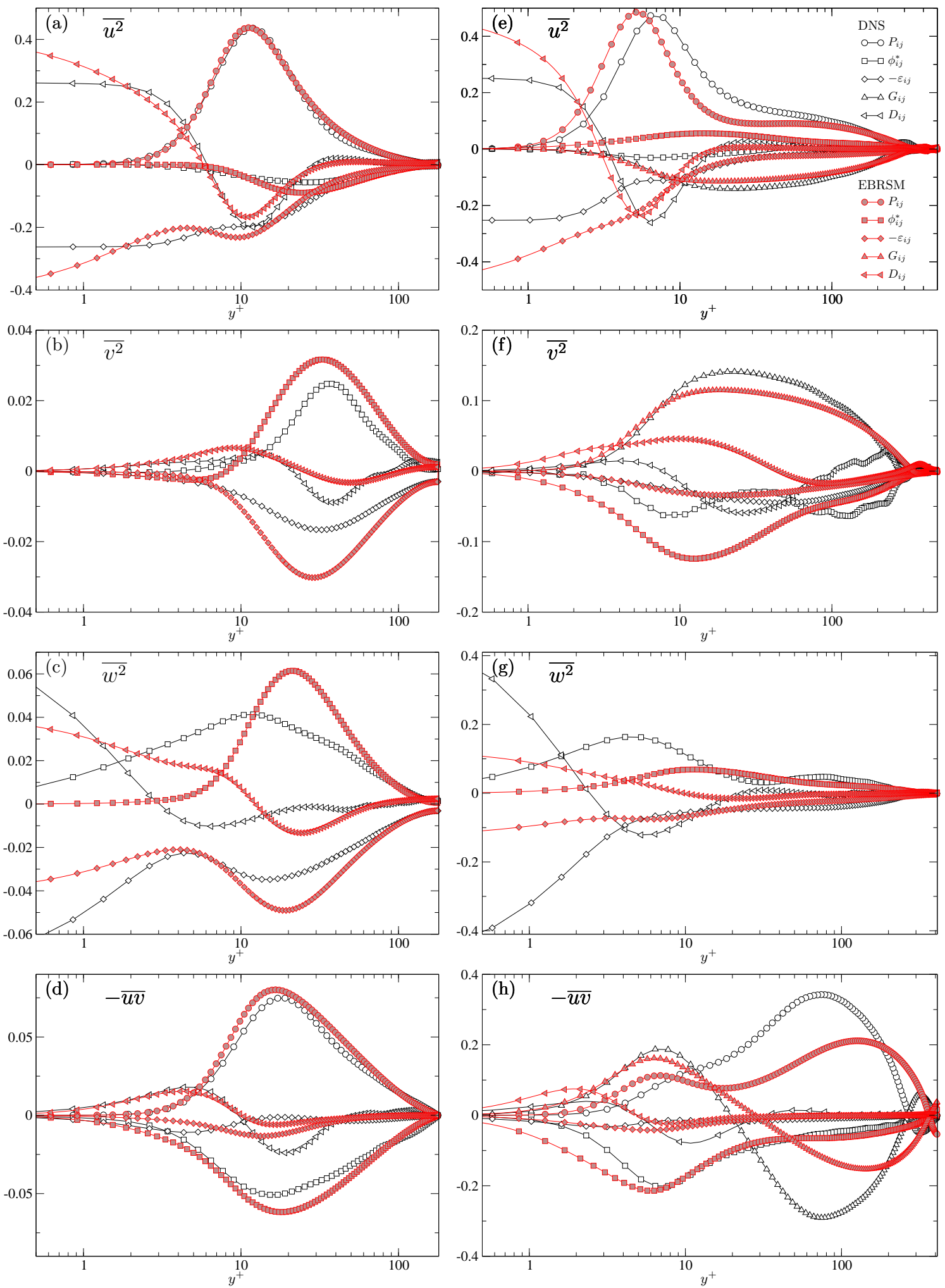

Figure 12: Budgets at $R e_{\tau}=180$ of: $(\mathrm{a}, \mathrm{e}) \overline{u^{2}} ;(\mathrm{b}, \mathrm{f}) \overline{v^{2}} ;(\mathrm{c}, \mathrm{g}) \overline{w^{2}}$ and $(\mathrm{d}, \mathrm{h})-\overline{u v}$. (a,b,c,d) non-rotating case; (e,f,g,h) case $R o=0.98$. Comparison with the DNS data of Mansour et al. (1988) (non-rotating case) and Grundestam et al. (2008) (rotating case). $P_{i j}=$ production; $\phi_{i j}^{*}=$ velocity-pressure gradient correlation; $\varepsilon_{i j}=$ dissipation; $G_{i j}=$ Coriolis redistribution; $D_{i j}=$ diffusion (molecular+turbulent). Data are in wall units, based on the anticyclonic friction velocity, and $y^{+}=0$ corresponds to the anticyclonic wall. For the sake of clarity, zero terms are not plotted $\left(P_{22}, P_{33}\right.$ and $\left.G_{33}\right)$ and the budget of $-\overline{u v}$ is plotted rather than that of $\overline{u v}$, such that terms located on the upper half of the plots represent a gain (plotted terms are thus $-P_{12},-\phi_{12}^{*}, \varepsilon_{12},-G_{12}$ and $-D_{12}$ ). 


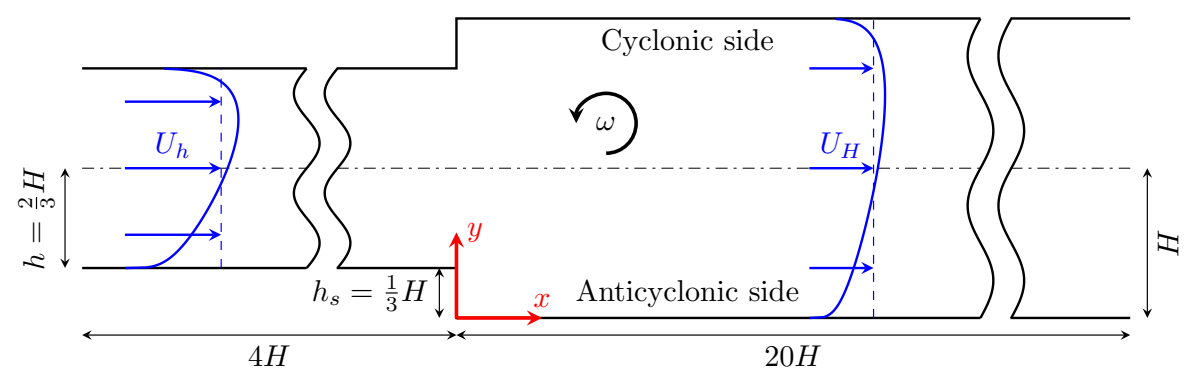

Figure 13: Schematic view of the rotating channel with sudden expansion.

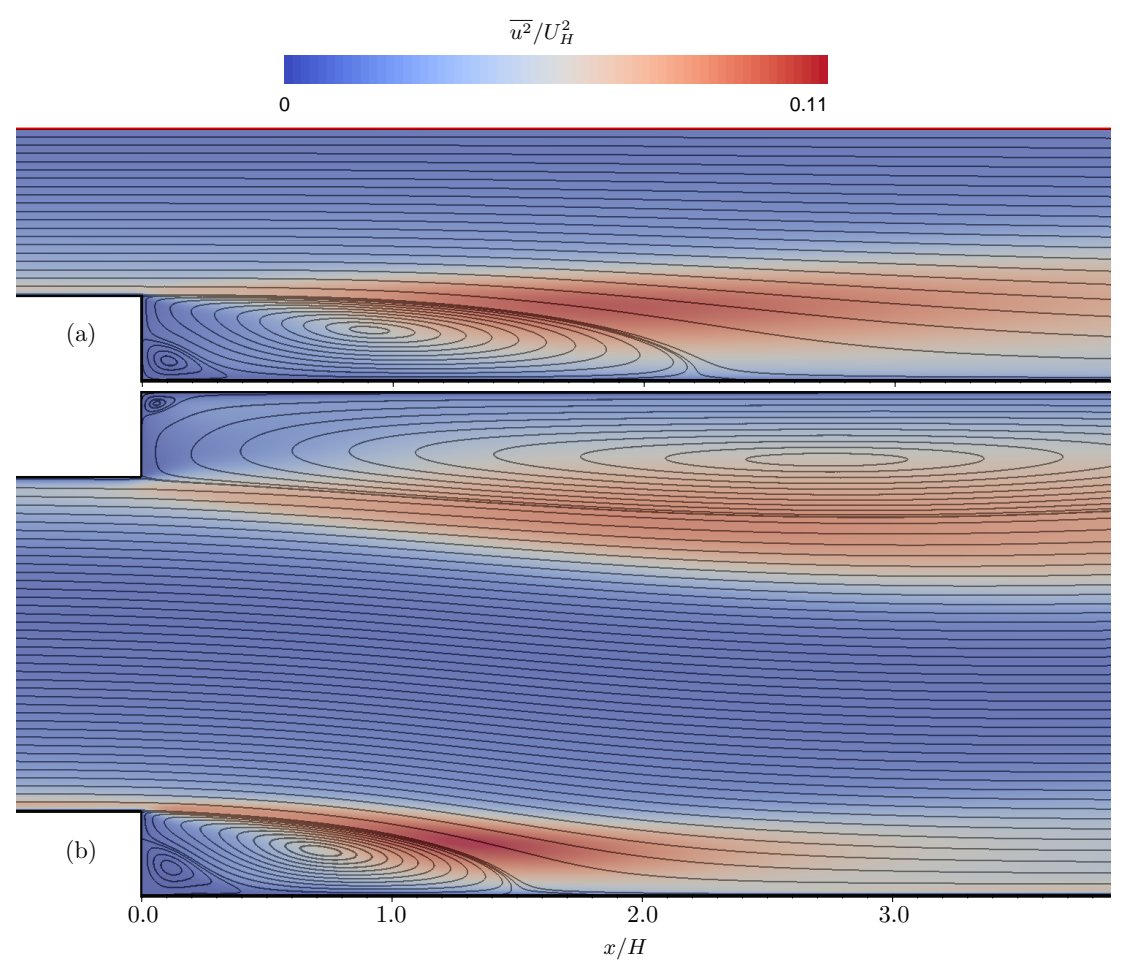

Figure 14: Channel with sudden expansion. Streamlines and isocontours of $\overline{u^{2}} / U_{H}^{2}$. (a) Non-rotating case; (b) Rotating case.

can be safely applied to more complex flows. Next section investigates the performance of the model in such a flow.

\section{Rotating channel flow with sudden expansion}

The case of a channel flow with sudden expansion under spanwise rotation is now investigated, corresponding to the very recent DNS data of Lamballais (2014). This geometrically simple configuration is relevant to the study of the influence of rotation on separated regions, which is encountered in turbomachinery applications or in geophysical fluid dynamics. Despite their challenging character for turbulence models and its practical importance, this type of flows has been the subject of only very few experimental (Rothe and Johnston, 1979; Visscher and Anders- son, 2011), DNS (Barri and Andersson, 2010; Lamballais, 2014), and turbulence modelling (Nilsen and Andersson, 1990; Iaccarino et al., 1999; Viswanathan and Tafti, 2007) studies. To the knowledge of the author, sudden-expansion flows with spanwise rotation have never been investigated with second moment closures.

The flow configuration, described in Fig. 13, is characterized by three non-dimensional numbers: the expansion ratio $E_{r}=H / h$, where $h$ and $H$ are the half-heights of the downstream and upstream channels, respectively; the Reynolds number $R e=h U_{h} / \nu=H U_{H} / \nu$, where $U_{h}$ and $U_{H}$ are the bulk velocities in the downstream and upstream channels, respectively; and the rotation number $R o=2 \omega H / U_{H}$, where $\omega$ is the angular velocity of the spanwise rotation. Here, these parameters are chosen to match 

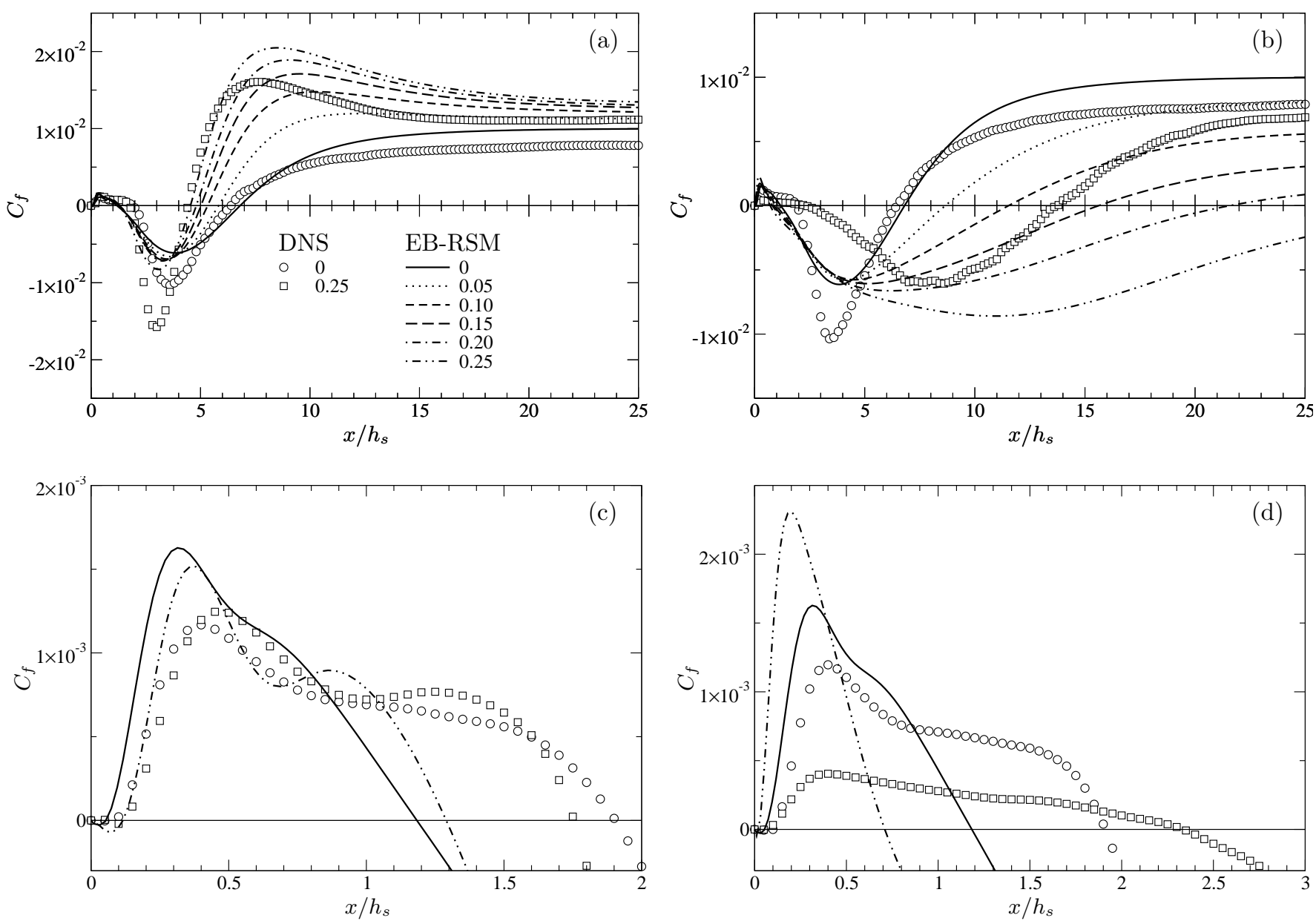

Figure 15: Channel with sudden expansion. Evolution with the rotation number of the $C_{f}$ distribution after the expansion. (a) Anticyclonic side; (b) Cyclonic side; (c,d) Enlargements of (a) and (b), respectively, showing the secondary bubbles (for the sake of clarity, only the cases $R o=0$ and $R o=0.25$ are shown).

the configuration of Lamballais (2014): $E_{r}=3 / 2$ (the step height is thus $\left.h_{s}=\left(E_{r}-1\right) h=h / 2=H / 3\right) ; R e=5000$; $R o=0$ and $R o=0.25$. In addition, in order to better describe the evolution of the flow with the rotation rate, four intermediate rotation numbers are computed: $R o=0.05$, $0.10,0.15$ and 0.20 .

Computations are performed with the open-source CFD solver Code_Saturne ${ }^{3}$, developed by EDF (Archambeau et al., 2004; Fournier et al., 2011), based on the finite volume method in cell-centered collocated arrangement, using a second-order upwind-biased differencing scheme. Inlet boundary conditions are generated by a precursor computation in a fully-developed (periodic) channel flow (see section 5), which is consistent with the recycling method used in the DNS. The inlet and outlet boundaries are located $4 H=12 h_{s}$ upstream and $20 H=60 h_{s}$ downstream of the expansion, respectively, which was found fully sufficient in preliminary tests.

Five different structured, 2D, meshes were built us-

\footnotetext{
${ }^{3}$ http://code-saturne.org
}

ing the open-source platform Salome ${ }^{4}$, in order to ensure a grid-converged solution. As usual with the EB-RSM (Manceau and Hanjalić, 2002), it was observed that maintaining the first discretization point nearby the wall below $y^{+}=1$ is sufficient. The influence of the refinement in the separated shear layer was also carefully checked and, owing to the relatively low-cost character of the computations, the finest mesh, consisting of 95000 cells, was used for all the computations presented below.

Fig. 14 shows the streamlines for the two cases $R o=0$ and 0.25 , in order to illustrate the main effect due to rotation in this type of flows, i.e., the shortening and lengthening of the recirculation bubbles on the anticyclonic and cyclonic sides, respectively. Fig. 15 shows the friction coefficient $C_{f}=\tau_{w} /\left(\frac{1}{2} \rho U_{H}^{2}\right)$ on the two walls, for the five values of the rotation number $R o$. The evolution of the recirculation lengths with the rotation rate, extracted from this figure, is plotted in Fig. 16. The model reproduces the monotonic shortening of the recirculation bubble on the anticyclonic side observed by Rothe and Johnston (1979).

\footnotetext{
${ }^{4}$ http://www.salome-platform.org
} 


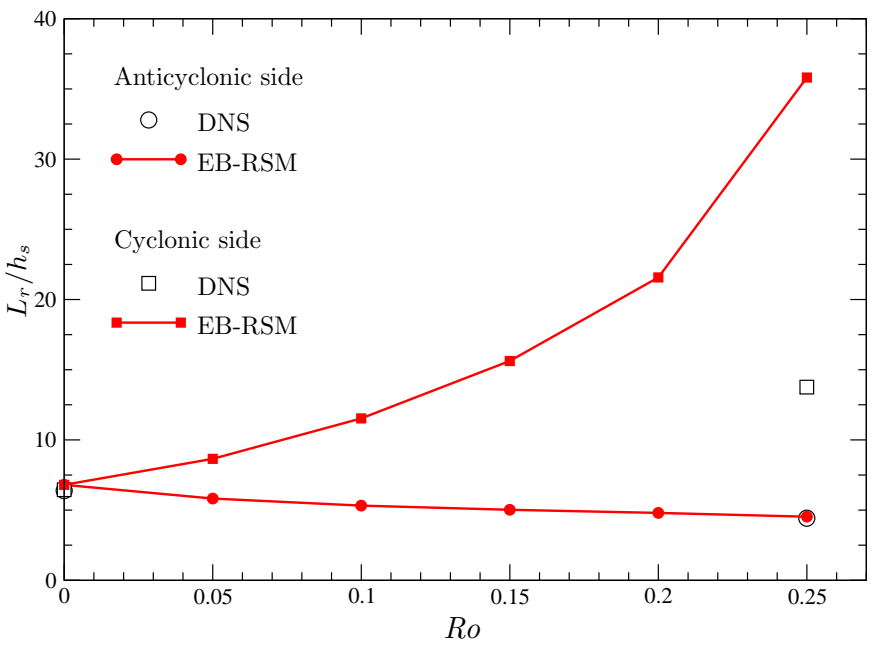

Figure 16: Channel with sudden expansion. Evolution of the reattachment length with the rotation number.

For the non-rotating case, the model gives a recirculation length $L_{r}=6.80 h_{s}$, which is only $6 \%$ above the DNS value $L_{r}=6.42 \pm 0.03$ (the error bar here comes from a residual asymmetry in the DNS results). For $R o=0.25$, the model shows a $33 \%$ reduction of the recirculation bubble $\left(L_{r}=4.53 h_{s}\right)$, which is to be compared to $31 \%$ for the DNS $\left(L_{r}=4.42 h_{s}\right)$. In contrast, it can be seen in Fig. $15 \mathrm{~b}$ that the model is, as already observed in section 5 , oversensitive to rotation on the cyclonic side. Whereas the DNS exhibits an increase of the recirculation length by a factor 3.1, the model predicts a factor 5.2. An interpolation of the results of Fig. 16 suggests that the model yields the same recirculation length as the DNS for a rotation number reduced by a factor about 2 .

Fig. 15a shows that the shape of the $C_{f}$ distribution on the anticyclonic side, for the non-rotating and the rotating cases, is correctly reproduced. The intensification with the rotation rate of the backflow is predicted, although the intensity is significantly underestimated by the model. After reattachment, the model overestimates the friction coefficient, due to too slow a recovery of the fully developed channel flow profile.

Figs. $15 \mathrm{c}$ and $15 \mathrm{~d}$ show that the $C_{f}$ distributions given by both the DNS and the EB-RSM computation exhibit two additional zero crossing, inside the main recirculation bubble, which are the footprints of secondary and tertiary recirculation bubbles. For the non-rotating case, the extents of these bubbles given by the DNS are $1.9 h_{s}$ and $0.05 h_{s}$, respectively, and $1.2 h_{s}$ and $0.05 h_{s}$ for the model. For the case $R o=0.25$, the DNS shows a decrease of the size of the secondary bubble on the anticyclonic side and an increase on the cyclonic side, while the model predicts opposite trends. In contrast, for the tertiary bubble, the model correctly predicts an increase on the anticyclonic side and a decrease on the cyclonic side.

The globally correct predictions of the model on the anticyclonic side seen in Fig. 15a, albeit with a slight over- estimation of the recirculation and recovery lengths and a significant underestimation of the backflow, are also visible in Fig. 17. A striking feature is the incorrect deceleration of the flow in the core region $(0.25 H<y<1.25 H)$ after the reattachment on the anticyclonic side. It clearly appears that this is a side effect of the strong overestimation of the backflow and of the size of the recirculation bubble on the cyclonic side: the negative mass flow rate is overestimated in the cyclonic recirculation region, and does not change sign after the location where DNS predicts the reattachment, such that, in order to preserve the global mass flow rate, the mean streamwise velocity is overestimated in the core region.

Figs. 18 and 19 show the turbulent kinetic energy and shear stress profiles, respectively, for the two cases $R o=0$ and $R o=0.25$. Both quantities are very satisfactorily reproduced for the non-rotating case, in particular before the expansion and in the recovery region, but, as usual with RANS models (see, for instance, Jakirlić and Maduta, 2014), their amplitudes are underestimated in the separated shear layer. On the anticyclonic side, the unstable character of the flow is correctly reproduced, leading to an increase of the turbulent energy and shear stress amplitudes, although the underestimation in the separated shear layer observed for the non-rotating case remains. On the cyclonic side, predictions are, as already noted in Fig. 17b, not satisfactory. In the region just after the expansion $(x / H<1), k$ and $\overline{u v}$ are significantly overestimated in the separated shear layer, yielding too strong an entrainment, which is at the origin of the too intense backflow in the cyclonic recirculation region observed in Fig. 17b. Downstream of the location $x / H=1$, the stabilization effect of rotation is, on the contrary, overestimated by the model, leading to the significant downstream shift of the reattachment point.

Fig. 20 compares the normal Reynolds stress profiles in the non-rotating and rotating cases. For the sake of clarity, only 4 profiles are plotted, located upstream of the expansion $(x / H=-1)$, across the recirculation bubbles $(x / H=0.5)$, after the non-rotating and anticyclonic reattachment points $(x / H=2)$ and further in the recovery region $(x / H=4)$. Note that on the cyclonic side, the last three profiles cross the recirculation bubble. In the non-rotating case, the predictions are globally satisfactory. It can be seen that the underestimation of the turbulent energy in the separated shear layer is mainly due to the streamwise component $\overline{u^{2}}$. In the recirculation bubble, a plateau of $\overline{u^{2}}$ is observed that is correctly reproduced by the model. Whereas $\overline{u^{2}}$ is better predicted than $\overline{v^{2}}$ and $\overline{w^{2}}$ just after reattachment, the opposite is observed in the downstream recovery region. For the rotating case, it is first observed that the Reynolds stresses are very satisfactory in the incoming channel. After the expansion, on the anticyclonic side, the anisotropy is globally very well reproduced, in particular in the recovery region. However, in the lower half of the core region $(0.25 H<y<0.75 H)$, the Reynolds stresses go too rapidly to zero. Due to the 


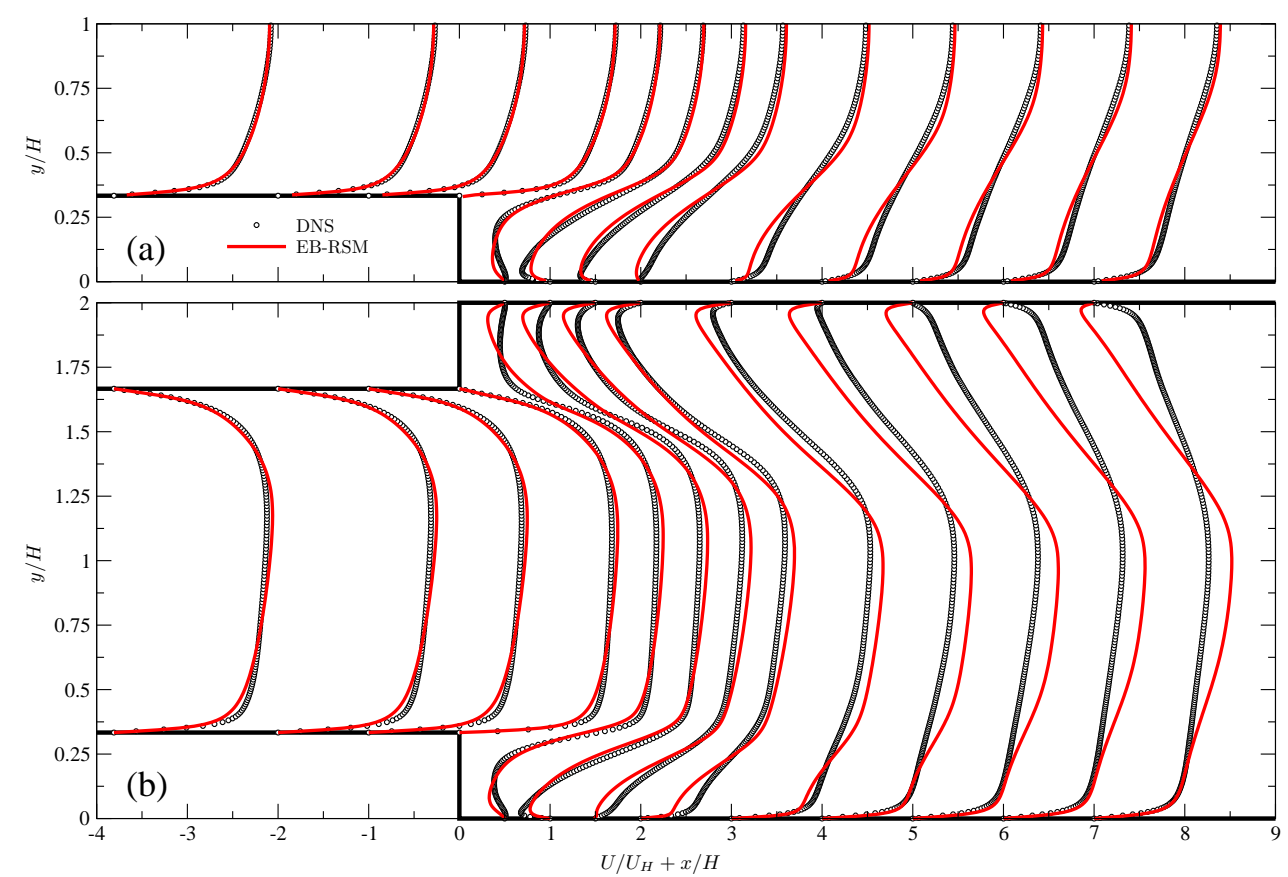

Figure 17: Channel with sudden expansion. Mean streamwise velocity profiles. (a) Non-rotating case; (b) Rotating case.

large amplitude of the underprediction, it is believed that it cannot be simply attributed to an underestimation of turbulent diffusion: this is to be traced to the misprediction of the streamwise velocity in this region (Fig. 17b). Indeed, as mentioned above, the overestimated recirculation length on the cyclonic side is at the origin of a significant underestimation of the deceleration in the core region. A deceleration yields a transfer of energy from the mean flow to turbulence through the production term $-\overline{u^{2}} \partial U / \partial x$ in the $\overline{u^{2}}$-equation, which helps maintaining the turbulent energy level. Although the DNS Reynolds stress budgets are not available, it can be thus conjectured that the underestimated deceleration is the main reason for the low level of $\overline{u^{2}}$ in the EB-RSM computation. Due to the pressure and Coriolis redistributions of energy among the components, $\overline{v^{2}}$ and $\overline{w^{2}}$ are in turn affected by this mechanism.

In conclusion, the EB-RSM is globally able to reproduce the main mechanisms driving the modification of the flow in a channel with sudden expansion. The observed discrepancies concerning the recirculation length on the cyclonic side and the tendency to laminarize in the core region, associated to an incorrect deceleration of the flow, can be attributed to the erroneous sensitivity of the model to rotation on the cyclonic side. This weakness, already pointed out in the case of a rotating channel without expansion in section 5, constitutes the main possibility of improvement for this type of flows for the future.

\section{Conclusion}

The various modifications of the original Elliptic Blending Reynolds stress model (EB-RSM) of Manceau and
Hanjalić (2002) proposed by several authors, and successfully applied to many complex configurations, have been revisited in order to formulate a reference EB-RSM. The ingredients of the model concerned with these modifications are the velocity-pressure gradient correlation tensor, either the near-wall or the weakly inhomogeneous formulations, the blending function used to continuously switch between these two formulations, and the generation term in the dissipation equation. Theoretical arguments and comparisons with DNS data lead to the following conclusions:

(i) The inclusion or not of the nonlinear term in the slow part of the SSG model, used in the weakly inhomogeneous regions, does not have a significant influence on the results. The use of this term is consequently not recommended, for numerical stability reasons.

(ii) In the original model, the near-wall formulation $\phi_{i j}^{w}$ for the velocity-pressure gradient correlation tensor does not lead to the correct asymptotic behaviour of the components $\overline{u v}$ and $\overline{v w}$, where $v$ is the wallnormal fluctuating velocity. The alternative formulation that corrects this behaviour, initially discarded by Manceau and Hanjalić (2002) based on channel flow results, and reintroduced by Törnblom and Johansson (2007), does not yield a correct prediction of the budgets of these Reynolds stress components.

(iii) The original formulation of the blending function $f_{\phi}=$ $k \alpha$ does not reproduce satisfactorily the friction coefficient in a channel flow over the range of friction Reynolds number for which DNS data are available $\left(180 \leq R e_{\tau} \leq 4200\right)$ and does not behave correctly 


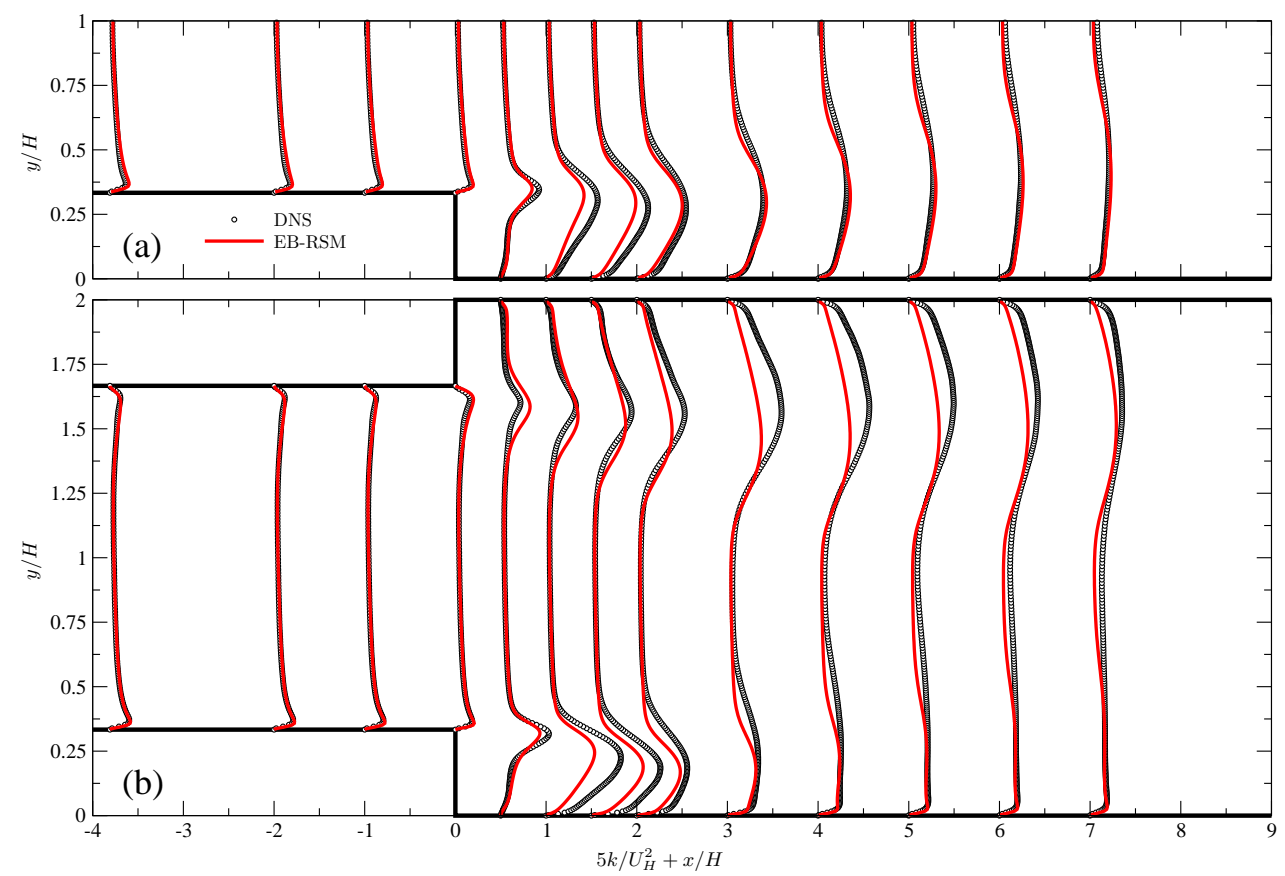

Figure 18: Channel with sudden expansion. Turbulent kinetic energy profiles. (a) Non-rotating case; (b) Rotating case.

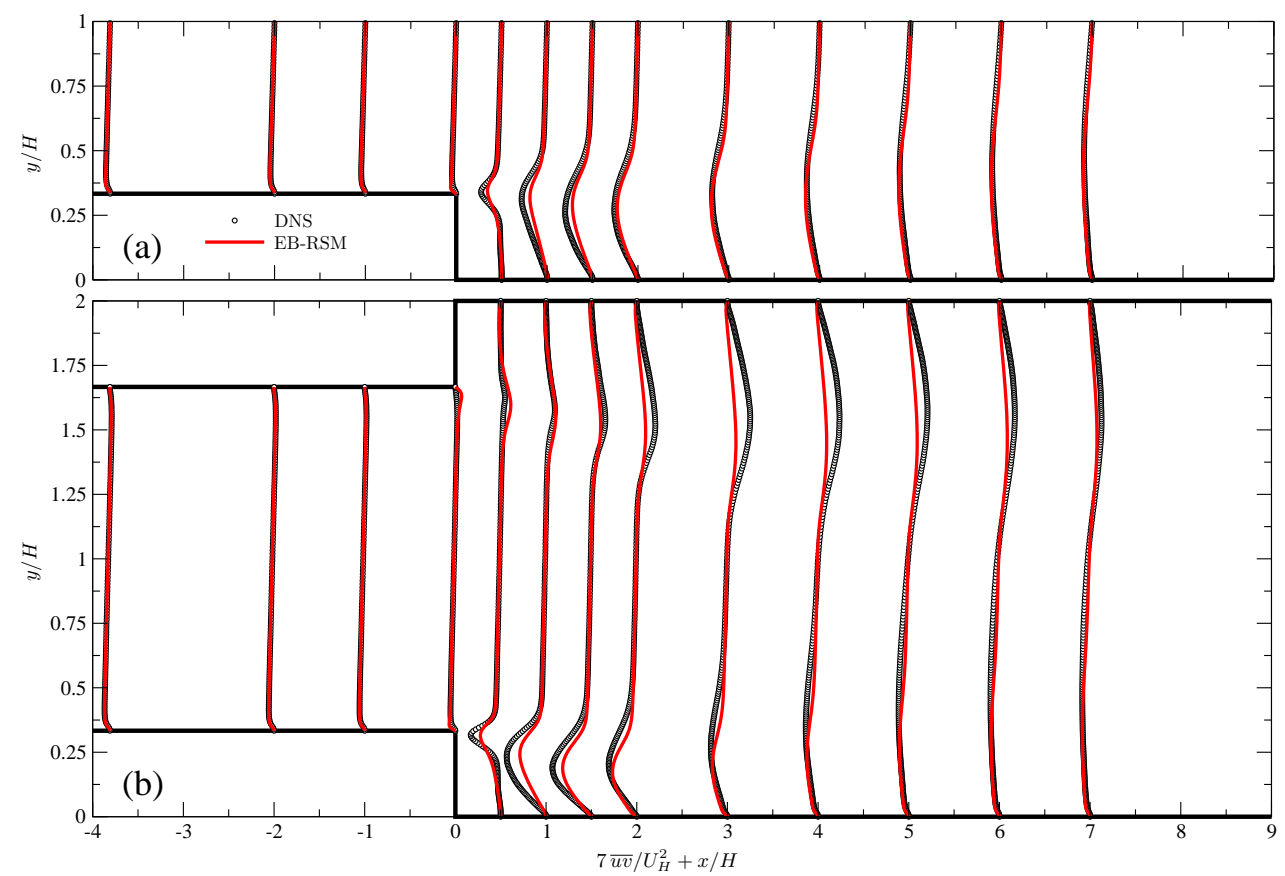

Figure 19: Channel with sudden expansion. Shear stress $(\overline{u v})$ profiles. (a) Non-rotating case; (b) Rotating case. 


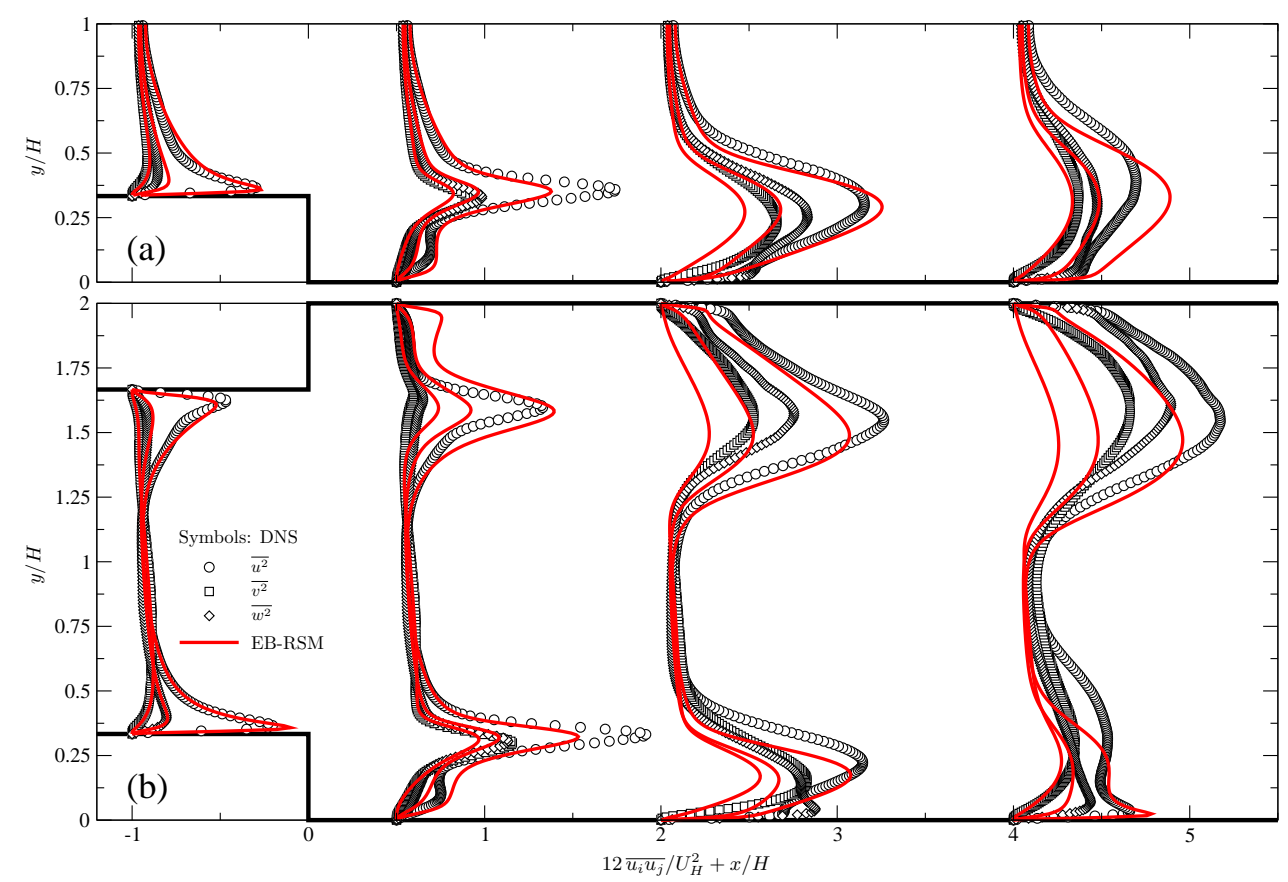

Figure 20: Channel with sudden expansion. Normal stress $\left(\overline{u^{2}}, \overline{v^{2}}, \overline{w^{2}}\right)$ profiles. (a) Non-rotating case; (b) Rotating case.

in rotating channels. The formulations $f_{\phi}=\alpha^{2}$ and $f_{\phi}=\alpha^{3}$ yield very similar results, and the latter is preferred based on the theoretical argument that it satisfies the exact asymptotic behaviour of the crucial term $\phi_{22}^{*}-\varepsilon_{22}$.

(iv) The various formulations used to account for the $P_{\varepsilon 3}$ term in the exact dissipation equation yield very similar results if coefficients are properly calibrated. The version using a variable coefficient $C_{\varepsilon 1}\left(1+A_{1}(1-\right.$ $\left.f_{\phi}\right) P(\varepsilon)$ is preferred to favour numerical robustness.

These conclusions lead to the reference EB-RSM given in Appendix C. Although heat transfer is beyond the scope of the present article, the model can be associated with a variety of models for the turbulent heat fluxes, ranging from the Elliptic Blending Generalized Gradient Hypothesis (EB-GGDH), well adapted to the forced convection regime (Dehoux et al., 2012; Benhamadouche et al., 2012), to the full Elliptic Blending Differential Flux Model (Shin et al., 2008; Dehoux, 2012), and including the Elliptic Blending Algebraic Flux Model (Dehoux et al., 2012).

The performance of the reference EB-RSM was investigated in detail in cases with spanwise rotation. The model does not require any adaptation to rotation in addition to the standard inclusion of the Coriolis term and the replacement of the mean vorticity tensor by the absolute mean vorticity tensor. In spanwise rotating channel flows, the model correctly reproduces the effects of rotation, in particular on the anticyclonic (pressure) side, but shows an inaccurate sensitivity to rotation on the cyclonic (suction) side. Although many of the good properties of the model in rotating cases are to be attributed to the SSG model on which it relies far from the wall, the Elliptic Blending strategy appears relevant to extend this model to nearwall regions. As a corollary, some discrepancies between the EB-RSM and DNS results are also inherited from the SSG model, in particular the slight under-prediction of the slope of the linear velocity profile in the core region and the delayed laminarization at high rotation numbers, which are linked to the slight over-prediction of the bifurcation threshold $\omega / S=0.5$ for sheared homogeneous turbulence with system rotation.

In the case with separation, the conclusions are very similar. EB-RSM predictions are globally very satisfactory, in particular for the cases without or with anticyclonic rotation, yielding an excellent reproduction of the recirculation length. However, consistent with the rotating channel case, the cyclonic region is found over-sensitive to rotational effects, leading to an overestimation of the recirculation length. This misprediction has a significant impact on the rest of the domain, in particular the core region, which experiences an overestimation of the mean streamwise velocity to compensate for the loss of flow rate on the cyclonic side. Since additional corrections of the model, via the dissipation equation, used in rotating homogeneous turbulence in order to inhibit the energy cascade, were found detrimental to the prediction of the cyclonic side in rotating channel flows, the improvement of the sensitivity to rotation in this region remains an open issue.

\section{Acknowledgements}

The present work owes much to the influence of $\mathrm{K}$. Hanjalić, co-author of the original EB-RSM, and B. Laun- 
der, who, among others, have pioneered modern turbulence modelling and inspired subsequent generations of researchers. O. Grundestam and S. Wallin are gratefully acknowledged for providing their DNS data. Special thanks are due to E. Lamballais for making his data available and for many friendly discussions about the present topic and many others during years spent in Poitiers. I am also embedded to T.B. Gatski for his comments about Appendix $\mathrm{B}$ and for our friendly collaboration over the years. This article is dedicated to the memory of our colleagues, C. Fourment and J. Delville, who recently passed away.

\section{Appendix A. Asymptotic behaviour of the terms of the Reynolds-stress budget}

This appendix provides, in the case of an incompressible flow, the asymptotic behaviour in the vicinity of a solid wall of the terms of the Reynolds-stress budget

$-C_{i j}+D_{i j}^{\nu}+D_{i j}^{T}+\phi_{i j}^{*}+P_{i j}-\varepsilon_{i j}=0$,

where $C_{i j}, D_{i j}^{\nu}, D_{i j}^{T}, \phi_{i j}^{*}, P_{i j}$ and $\varepsilon_{i j}$ denote convection, viscous diffusion, turbulent diffusion, velocity-pressure gradient correlation, production and dissipation, respectively.

The reference frame can, without loss of generality, be chosen such that the $y$-direction is locally normal to the wall. The Taylor series expansion of the mean velocities $U, V, W$ and the fluctuating velocities $u, v, w$ and pressure $p$ reads

$$
\begin{array}{cc}
U= & A_{1}(x, z, t) y+A_{2}(x, z, t) y^{2}+\mathcal{O}\left(y^{3}\right), \\
V= & B_{2}(x, z, t) y^{2}+\mathcal{O}\left(y^{3}\right), \\
W= & C_{1}(x, z, t) y+C_{2}(x, z, t) y^{2}+\mathcal{O}\left(y^{3}\right), \\
u= & a_{1}(x, z, t) y+a_{2}(x, z, t) y^{2}+\mathcal{O}\left(y^{3}\right),(\mathrm{A} .2) \\
v= & b_{2}(x, z, t) y^{2}+\mathcal{O}\left(y^{3}\right), \\
w= & c_{1}(x, z, t) y+c_{2}(x, z, t) y^{2}+\mathcal{O}\left(y^{3}\right), \\
p=p_{0}(x, z, t)+p_{1}(x, z, t) y+p_{2}(x, z, t) y^{2}+\mathcal{O}\left(y^{3}\right),
\end{array}
$$

where $A_{i}, B_{i}$ and $C_{i}$ are deterministic functions, and $a_{i}$, $b_{i}, c_{i}$ and $p_{i}$ are stochastic variables.

Using the identities $\partial p_{0} / \partial x=2 \rho \nu a_{2}, \partial p_{0} / \partial z=2 \rho \nu c_{2}, p_{1}=$ $2 \rho \nu b_{2}$ and $p_{2}=3 \rho \nu b_{3}$ obtained from the near-wall balance of the fluctuating Navier-Stokes equations, the Taylorseries expansions of the terms in Eq. (A.1) lead to the dominant terms shown in table A.3.

\section{Appendix B. Derivation of the near-wall form $\phi_{i j}^{w}$}

The purpose of the present appendix is to derive a general form for the symmetric tensor $\boldsymbol{\Phi}^{w} / \varepsilon$ as a function of the anisotropy tensor $\mathbf{b}$ and the non-dimensional, unit vector $\mathbf{n}$. The general theorems of the theory of invariants applied below can be found in Spencer (1971) or, in a summarized form, more accessible to fluid dynamicist, in Deville and Gatski (2012).

\section{Appendix B.1. Integrity basis}

The first step towards a polynomial representation of $\boldsymbol{\Phi}^{w} / \varepsilon$ is the identification of the integrity basis associated with the second-order tensor $\mathbf{b}$ and the absolute vector $\mathbf{n}$, i.e., the minimal set of functions of $\mathbf{b}$ and $\mathbf{n}$ necessary to generate all the polynomial invariants of $\mathbf{b}$ and $\mathbf{n}$ under the full orthogonal group of transformations.

The integrity basis consists of irreducible invariants formed using $\mathbf{b}$ by itself, which can be extracted from table 2.1 of Deville and Gatski (2012),

$\{\mathbf{b}\} ;\left\{\mathbf{b}^{2}\right\} ;\left\{\mathbf{b}^{3}\right\}$

and additional invariants of the form

$n_{i} \Pi_{i j}^{(k)} n_{j}$,

where $\Pi^{(k)}=\Pi_{i i}^{(k)}$ are invariants listed in table 2.3a of Deville and Gatski (2012),

$n_{i} \delta_{i j} n_{j} ; n_{i} b_{i j} n_{j} ; n_{i} b_{i k} b_{k j} n_{j}$.

Introducing the second-order tensor $\mathbf{N}=\mathbf{n} \otimes \mathbf{n}$, the general integrity basis for one symmetric tensor and one absolute vector then reads

$\{\mathbf{b}\} ;\left\{\mathbf{b}^{2}\right\} ;\left\{\mathbf{b}^{3}\right\} ;\{\mathbf{N}\} ;\{\mathbf{b N}\} ;\left\{\mathbf{b}^{2} \mathbf{N}\right\}$.

Here, using the particular features $\{\mathbf{b}\}=0$ and $\{\mathbf{N}\}=1$, the basis reduces to

$\left\{\mathbf{b}^{2}\right\} ;\left\{\mathbf{b}^{3}\right\} ;\{\mathbf{b} \mathbf{N}\} ;\left\{\mathbf{b}^{2} \mathbf{N}\right\}$.

It is worth mentioning that this procedure is equivalent to constructing the integrity basis for two symmetric tensors (here, $\mathbf{b}$ and $\mathbf{N}=\mathbf{n} \otimes \mathbf{n}$ ) and to discard terms of order two or higher in $\mathbf{N}$. This method is more familiar to the turbulence modelling community, since it is similar to that applied in the framework of the derivation of explicit algebraic models based on the symmetric tensor $\mathbf{S}$ and the skew-symmetric tensor W (Pope, 1975). Again, table 2.1 of Deville and Gatski (2012) gives the integrity basis

$\{\mathbf{b}\} ;\left\{\mathbf{b}^{2}\right\} ;\left\{\mathbf{b}^{3}\right\} ;\{\mathbf{N}\} ;\left\{\mathbf{N}^{2}\right\} ;$

$\left\{\mathbf{N}^{3}\right\} ;\{\mathbf{b} \mathbf{N}\} ;\left\{\mathbf{b N}^{2}\right\} ;\left\{\mathbf{b}^{2} \mathbf{N}\right\} ;\left\{\mathbf{b}^{2} \mathbf{N}^{2}\right\}$,

which yields Eq. (B.4) after discarding invariants of order more than one in $\mathbf{N}$.

The reason why terms of order more than one are reducible lies in the fact that the determinant of the tensorial product of a vector with itself is zero, such that $\left\{\mathbf{N}^{3}\right\}$ is reducible,

$\left\{\mathbf{N}^{3}\right\}=\frac{3}{2}\{\mathbf{N}\}\left\{\mathbf{N}^{2}\right\}-\frac{1}{2}\{\mathbf{N}\}^{3}$.

Consequently, the Cayley-Hamilton theorem reduces to

$\mathbf{N}^{2}=\{\mathbf{N}\} \mathbf{N}-\frac{1}{2}\left(\{\mathbf{N}\}^{2}-\left\{\mathbf{N}^{2}\right\}\right)$,

which yields

$\left\{\mathbf{N}^{2}\right\}=\{\mathbf{N}\}^{2}$. 


\begin{tabular}{|c|c|c|c|c|c|c|}
\hline & $-C_{i j}$ & $D_{i j}^{\nu}$ & $D_{i j}^{T}$ & $\phi_{i j}^{*}$ & $P_{i j}$ & $-\varepsilon_{i j}$ \\
\hline$\overline{u^{2}}$ & $\mathcal{O}\left(y^{3}\right)$ & $2 \nu \overline{a_{1}^{2}}+12 \nu \overline{a_{1} a_{2}} y+\mathcal{O}\left(y^{2}\right)$ & $\mathcal{O}\left(y^{3}\right)$ & $-4 \nu \overline{a_{1} a_{2}} y+\mathcal{O}\left(y^{2}\right)$ & $\mathcal{O}\left(y^{3}\right)$ & $-2 \nu \overline{a_{1}^{2}}-8 \nu \overline{a_{1} a_{2}} y+\mathcal{O}\left(y^{2}\right)$ \\
\hline$\overline{v^{2}}$ & $\mathcal{O}\left(y^{5}\right)$ & $12 \nu \overline{b_{2}^{2}} y^{2}+40 \nu \overline{b_{2} b_{3}} y^{3}+\mathcal{O}\left(y^{4}\right)$ & $\mathcal{O}\left(y^{5}\right)$ & $-4 \nu \overline{b_{2}^{2}} y^{2}-16 \nu \overline{b_{2} b_{3}} y^{3}+\mathcal{O}\left(y^{4}\right)$ & $\mathcal{O}\left(y^{5}\right)$ & $-8 \nu \overline{b_{2}^{2}} y^{2}-24 \nu \overline{b_{2} b_{3}} y^{3}+\mathcal{O}\left(y^{4}\right)$ \\
\hline$\overline{w^{2}}$ & $\mathcal{O}\left(y^{3}\right)$ & $2 \nu \overline{c_{1}^{2}}+12 \nu \overline{c_{1} c_{2}} y+\mathcal{O}\left(y^{2}\right)$ & $\mathcal{O}\left(y^{3}\right)$ & $-4 \nu \overline{c_{1} c_{2}} y \mathcal{O}\left(y^{2}\right)$ & $\mathcal{O}\left(y^{3}\right)$ & $-2 \nu \overline{c_{1}^{2}}-8 \nu \overline{c_{1} c_{2}} y+\mathcal{O}\left(y^{2}\right)$ \\
\hline$\overline{u v}$ & $\mathcal{O}\left(y^{4}\right)$ & $6 \nu \overline{a_{1} b_{2}} y+\left(12 \nu \overline{a_{1} b_{3}}+12 \nu \overline{a_{2} b_{2}}\right) y^{2}+\mathcal{O}\left(y^{3}\right)$ & $\mathcal{O}\left(y^{4}\right)$ & $-2 \nu \overline{a_{1} b_{2}} y-\left(6 \nu \overline{a_{1} b_{3}}+4 \nu \overline{a_{2} b_{2}}\right) y^{2}+\mathcal{O}\left(y^{3}\right)$ & $\mathcal{O}\left(y^{4}\right)$ & $-4 \nu \overline{a_{1} b_{2}} y-\left(6 \nu \overline{a_{1} b_{3}}+8 \nu \overline{a_{2} b_{2}}\right) y^{2}+\mathcal{O}\left(y^{3}\right)$ \\
\hline$\overline{u w}$ & $\mathcal{O}\left(y^{3}\right)$ & $2 \nu \overline{a_{1} c_{1}}+\left(6 \nu \overline{a_{1} c_{2}}+6 \nu \overline{a_{2} c_{1}}\right) y+\mathcal{O}\left(y^{2}\right)$ & $\mathcal{O}\left(y^{3}\right)$ & $\left(-2 \nu \overline{a_{1} c_{2}}-2 \nu \overline{a_{2} c_{1}}\right) y+\mathcal{O}\left(y^{2}\right)$ & $\mathcal{O}\left(y^{3}\right)$ & $-2 \nu \overline{a_{1} c_{1}}\left(-4 \nu \overline{a_{1} c_{2}}-4 \nu \overline{a_{2} c_{1}}\right) y+\mathcal{O}\left(y^{2}\right)$ \\
\hline$\overline{v w}$ & $\mathcal{O}\left(y^{4}\right)$ & $6 \nu \overline{b_{2} c_{1}} y+\left(12 \nu \overline{b_{3} c_{1}}+12 \nu \overline{b_{2} c_{2}}\right) y^{2}+\mathcal{O}\left(y^{3}\right)$ & $\mathcal{O}\left(y^{4}\right)$ & $-2 \nu \overline{b_{2} c_{1}} y-\left(6 \nu \overline{b_{3} c_{1}}+4 \nu \overline{b_{2} c_{2}}\right) y^{2}+\mathcal{O}\left(y^{3}\right)$ & $\mathcal{O}\left(y^{4}\right)$ & $-4 \nu \overline{b_{2} c_{1}} y-\left(6 \nu \overline{b_{3} c_{1}}+8 \nu \overline{b_{2} c_{2}}\right) y^{2}+\mathcal{O}\left(y^{3}\right)$ \\
\hline
\end{tabular}

Table A.3: Dominant terms in the Taylor series expansion of the Reynolds stress budgets.

Combining Eqs. (B.7) and (B.8) simply leads to

$\left\{\mathbf{N}^{3}\right\}=\{\mathbf{N}\}^{3}$.

Additionally, in the present case, $\mathbf{n}$ is a unit vector, such that $\{\mathbf{N}\}=1$, and

$\mathbf{N}^{2}=\mathbf{N}$.

\section{Appendix B.2. Polynomial representation of $\boldsymbol{\Phi}^{w} / \varepsilon$}

The method to build a polynomial representation of $\boldsymbol{\Phi}^{w} / \varepsilon$ consists in identifying the integrity basis for the invariants of $\mathbf{b}, \mathbf{n}$ and the arbitrary symmetric tensor $\boldsymbol{\Psi}$. Indeed, the invariant

$\mathcal{J}=\frac{1}{\varepsilon}\left\{\boldsymbol{\Phi}^{w} \boldsymbol{\Psi}\right\}$

is a polynomial of $\mathbf{b}, \mathbf{n}$ and $\boldsymbol{\Psi}$, such that a polynomial representation of $\boldsymbol{\Phi}^{w} / \varepsilon$ can be obtained by taking the derivative of $\mathcal{J}$ with respect to $\boldsymbol{\Psi}$. Since $\boldsymbol{\Phi}^{w} / \varepsilon$ is independent of $\boldsymbol{\Psi}$, the polynomial representation of $\mathcal{J}$ must be restricted to terms linear in $\boldsymbol{\Psi}$.

These terms are irreducible invariants formed from $\mathbf{b}$ and $\boldsymbol{\Psi}$ that are linear in $\boldsymbol{\Psi}$

$\{\boldsymbol{\Psi}\} ;\{\mathbf{b} \boldsymbol{\Psi}\} ;\left\{\mathbf{b}^{2} \boldsymbol{\Psi}\right\}$,

complemented by invariants of the form

$n_{i} \Psi_{i j} n_{j} ; n_{i} b_{i k} \Psi_{k j} n_{j} ; n_{i} b_{i k} b_{k l} \Psi_{l j} n_{j}$,

i.e.,

$\{\mathbf{N} \boldsymbol{\Psi}\} ;\{\mathbf{b} \mathbf{N} \boldsymbol{\Psi}\} ;\left\{\mathbf{b}^{2} \mathbf{N} \boldsymbol{\Psi}\right\}$.

Again, the same list of terms can be obtained from the irreducible invariants of $\mathbf{b}, \mathbf{N}$ and $\mathbf{\Psi}$, linear in $\mathbf{\Psi}$,

$\{\boldsymbol{\Psi}\} ;\{\mathbf{b} \boldsymbol{\Psi}\} ;\left\{\mathbf{b}^{2} \boldsymbol{\Psi}\right\} ;\{\mathbf{N} \boldsymbol{\Psi}\} ;\left\{\mathbf{N}^{2} \boldsymbol{\Psi}\right\} ;$

$\{\mathbf{b N} \boldsymbol{\Psi}\} ;\left\{\mathbf{b}^{2} \mathbf{N} \boldsymbol{\Psi}\right\} ;\left\{\mathbf{b N}^{2} \boldsymbol{\Psi}\right\} ;\left\{\mathbf{b}^{2} \mathbf{N}^{2} \boldsymbol{\Psi}\right\}$,

discarding invariants of order more than one in $\mathbf{N}$,

$\{\boldsymbol{\Psi}\} ;\{\mathbf{b} \boldsymbol{\Psi}\} ;\left\{\mathbf{b}^{2} \boldsymbol{\Psi}\right\} ;\{\mathbf{N} \boldsymbol{\Psi}\} ;\{\mathbf{b} \mathbf{N} \boldsymbol{\Psi}\} ;\left\{\mathbf{b}^{2} \mathbf{N} \boldsymbol{\Psi}\right\}$.

The polynomial representation of the symmetric tensor $\boldsymbol{\Phi}^{w} / \varepsilon$ can then be obtained as

$\frac{\phi_{i j}^{w}}{\varepsilon}=\frac{1}{2}\left(\frac{\phi_{i j}^{w}}{\varepsilon}+\frac{\phi_{j i}^{w}}{\varepsilon}\right)=\frac{1}{2}\left(\frac{\partial \mathcal{J}}{\partial \Psi_{i j}}+\frac{\partial \mathcal{J}}{\partial \Psi_{j i}}\right)$, such that it contains the terms

$\mathbf{I} ; \mathbf{b} ; \mathbf{b}^{2} ; \mathbf{N} ; \mathbf{b N}+\mathbf{N b} ; \mathbf{b}^{2} \mathbf{N}+\mathbf{N b}^{2}$.

Since $\boldsymbol{\Phi}^{w} / \varepsilon$ is traceless, it is convenient to introduce the traceless tensor $\mathbf{M}=\mathbf{N}-\frac{1}{3} \mathbf{I}$, and to recast the polynomial representation in the form

$$
\begin{aligned}
\frac{\Phi^{w}}{\varepsilon}= & \beta_{1} \mathbf{b}+\beta_{2} \mathbf{M}+\beta_{3}\left(\mathbf{b} \mathbf{M}+\mathbf{M} \mathbf{b}-\frac{2}{3}\{\mathbf{b} \mathbf{M}\} \mathbf{I}\right) \\
& +\beta_{4}\left(\mathbf{b}^{2}-\frac{1}{3}\left\{\mathbf{b}^{2}\right\} \mathbf{I}\right) \\
& +\beta_{5}\left(\mathbf{b}^{2} \mathbf{M}+\mathbf{M b}^{2}-\frac{2}{3}\left\{\mathbf{b}^{2} \mathbf{M}\right\} \mathbf{I}\right),
\end{aligned}
$$

The coefficients $\beta_{i}$ are polynomial invariants of the tensor $\mathbf{b}$ and the vector $\mathbf{n}$, and, as such, are polynomial functions of the terms of the integrity basis given by Eq. (B.5).

\section{Appendix C. The Elliptic Blending Reynolds-Stress Model}

Appendix C.1. Reference model

(a) Reynolds-stress transport equation:

$\frac{\mathrm{D} \overline{u_{i} u_{j}}}{\mathrm{D} t}=P_{i j}+G_{i j}+D_{i j}^{\nu}+D_{i j}^{T}+\phi_{i j}^{*}-\varepsilon_{i j}$,

where $P_{i j}, D_{i j}^{\nu}, D_{i j}^{T}, \phi_{i j}^{*}$ and $\varepsilon_{i j}$ stands for the production, the molecular diffusion, the turbulent diffusion, the velocity-pressure gradient correlation and the dissipation tensor, respectively. $G_{i j}=-2 \omega_{k}\left(\epsilon_{i k m} \overline{u_{j} u_{m}}+\right.$ $\epsilon_{j k m} \overline{u_{i} u_{m}}$ ) is the redistribution term arising from the Coriolis acceleration, where $\boldsymbol{\omega}$ is the rotation axial vector.

(b) Velocity-pressure gradient correlation:

$\phi_{i j}^{*}=\left(1-\alpha^{3}\right) \phi_{i j}^{w}+\alpha^{3} \phi_{i j}^{h}$,

which combines the SSG model (Speziale et al., 1991)

$$
\begin{aligned}
\phi_{i j}^{h}= & -\left(g_{1}+g_{1}^{*} \frac{P}{\varepsilon}\right) \varepsilon b_{i j}+\left(g_{3}-g_{3}^{*} \sqrt{b_{k l} b_{k l}}\right) k S_{i j} \\
& +g_{4} k\left(b_{i k} S_{j k}+b_{j k} S_{i k}-\frac{2}{3} b_{l m} S_{l m} \delta_{i j}\right) \\
& +g_{5} k\left(b_{i k} W_{j k}+b_{j k} W_{i k}\right),
\end{aligned}
$$

where

$b_{i j}=\frac{\overline{u_{i} u_{j}}}{2 k}-\frac{1}{3} \delta_{i j}$ 
$S_{i j}=\frac{1}{2}\left(\frac{\partial U_{i}}{\partial x_{j}}+\frac{\partial U_{j}}{\partial x_{i}}\right)$,

$W_{i j}=\frac{1}{2}\left(\frac{\partial U_{i}}{\partial x_{j}}-\frac{\partial U_{j}}{\partial x_{i}}\right)+\epsilon_{m j i} \omega_{m}$,

and the near-wall model

$$
\begin{aligned}
\phi_{i j}^{w}=- & 5 \frac{\varepsilon}{k}\left[\overline{u_{i} u_{k}} n_{j} n_{k}+\overline{u_{j} u_{k}} n_{i} n_{k}\right. \\
- & \left.\frac{1}{2} \overline{u_{k} u_{l}} n_{k} n_{l}\left(n_{i} n_{j}+\delta_{i j}\right)\right] .
\end{aligned}
$$

(c) Elliptic relaxation equation:

$\alpha-L^{2} \nabla^{2} \alpha=1$.

(d) Approximate wall normal vector:

$$
\mathbf{n}=\frac{\nabla \alpha}{\|\nabla \alpha\|} .
$$

(e) Turbulent diffusion:

$$
D_{i j}^{T}=\frac{\partial}{\partial x_{l}}\left(\frac{C_{\mu}}{\sigma_{k}} \overline{u_{l} u_{m}} T \frac{\partial \overline{u_{i} u_{j}}}{\partial x_{m}}\right) .
$$

(f) Dissipation tensor:

$$
\varepsilon_{i j}=\left(1-\alpha^{3}\right) \frac{\overline{u_{i} u_{j}}}{k} \varepsilon+\frac{2}{3} \alpha^{3} \varepsilon \delta_{i j} .
$$

(g) Time and length scales:

$$
\begin{aligned}
T & =\max \left(\frac{k}{\varepsilon}, C_{T}\left(\frac{\nu}{\varepsilon}\right)^{1 / 2}\right), \\
L & =C_{L} \max \left(\frac{k^{3 / 2}}{\varepsilon}, C_{\eta} \frac{\nu^{3 / 4}}{\varepsilon^{1 / 4}}\right) .
\end{aligned}
$$

(h) Dissipation equation:

$$
\begin{aligned}
\frac{\mathrm{D} \varepsilon}{\mathrm{D} t}= & \frac{C_{\varepsilon_{1}}^{\prime} P-C_{\varepsilon_{2}} \varepsilon}{T}+\frac{\partial}{\partial x_{l}}\left(\frac{C_{\mu}}{\sigma_{\varepsilon}} \overline{u_{l} u_{m}} T \frac{\partial \varepsilon}{\partial x_{m}}\right) \\
& +\nu \frac{\partial^{2} \varepsilon}{\partial x_{k} \partial x_{k}},
\end{aligned}
$$

where

$C_{\varepsilon_{1}}^{\prime}=C_{\varepsilon_{1}}\left[1+A_{1}\left(1-\alpha^{3}\right) \frac{P}{\varepsilon}\right]$.

(i) Coefficients:

$$
\begin{gathered}
g_{1}=3.4 ; g_{1}^{*}=1.8 ; g_{3}=0.8 ; g_{3}^{*}=1.3 ; g_{4}=1.25 ; g_{5}=0.4 ; \\
C_{\mu}=0.21 ; \sigma_{k}=1.0 ; C_{T}=6.0 ; \\
C_{L}=0.133 ; C_{\eta}=80.0 ; \\
C_{\varepsilon_{1}}=1.44 ; C_{\varepsilon_{2}}=1.83 ; A_{1}=0.065 ; \sigma_{\varepsilon}=1.15 .
\end{gathered}
$$

(j) Wall boundary conditions:

$$
U_{i}=0 ; \overline{u_{i} u_{j}}=0 ; \varepsilon=2 \nu \lim _{y \rightarrow 0} \frac{k}{y^{2}} ; \alpha=0 .
$$

\section{Appendix C.2. Other versions used in the present article}

Table C.4 provides a description of the various models used within the present article, in comparison with the reference model described above.

\section{References}

Archambeau, F., Méchitoua, N., Sakiz, M., 2004. Code Saturne: A Finite Volume Code for the Computation of Turbulent Incompressible flows - Industrial Applications. Int. J. on Finite Volume, Electronical edition: http://averoes.math.univparis13.fr/html ISSN 1634 (0655).

Bardina, J., Ferziger, J., Rogallo, R., 1985. Effect of rotation on isotropic turbulence: computation and modelling. J. Fluid Mech. 154, 321-336.

Bardina, J., Ferziger, J. H., Reynolds, W. C., 1983. Improved turbulence models based on large-eddy simulation of homogeneous, incompressible, turbulent flows. Report TF-19, Stanford University, California, USA.

Barri, M., Andersson, H., 2010. Turbulent flow over a backwardfacing step. Part 1. Effects of anti-cyclonic system rotation. J. Fluid Mech. 665, 382-417.

Belcher, S. E., Durbin, P. A., 1992. A second-order closure study of rotating channel flow without the use of 'damping functions'. CTR Manuscript 134, Center for Turbulence Research, Stanford University, CA, USA.

Benhamadouche, S., Afgan, I., Dehoux, F., Manceau, R., 2012. Unsteady RANS and large-eddy simulation of the flow and heat transfer in a wall bounded pin matrix. In: Proc. 7th Int. Symp. Turbulence, Heat Mass Transfer.

Bertoglio, J., 1982. Homogeneous turbulent field within a rotating frame. AIAA J. 20 (9), 1175-1181.

Billard, F., Laurence, D., 2012. A robust $k-\varepsilon-v^{2} / \mathrm{k}$ elliptic blending turbulence model applied to near-wall, separated and buoyant flows. Int. J. Heat Fluid Fl. 33 (1), 45-58.

Billard, F., Revell, A., Craft, T., 2012. Application of recently developed elliptic blending based models to separated flows. Int. J. Heat Fluid Fl. 35, 141-151.

Borello, D., Hanjalic, K., Rispoli, F., 2005. Prediction of cascade flows with innovative second-moment closures. J. Fluid Eng.-T. ASME 127 (6), 1059-1070.

Bradshaw, P., 1969. The analogy between streamline curvature and buoyancy in turbulent shear flow. J. Fluid Mech. 36, 177-191.

Bradshaw, P., Mansour, N. N., Piomelli, U., 1987. On Local Approximations of the Pressure--Strain Term in Turbulence Models. In: Proc. of the Summer Program. Center for Turbulence Research, Stanford University, CA, USA, pp. 159-164.

Brumley, B., Jirka, G., 1987. Near-surface turbulence in a grid-stirred tank. J. Fluid Mech. 183, 235-263.

Calmet, I., Magnaudet, J., 2003. Statistical structure of highReynolds-number turbulence close to the free surface of an openchannel flow. J. Fluid Mech. 474, 355-378.

Campagne, G., Cazalbou, J.-B., Joly, L., Chassaing, P., 2009. The structure of a statistically steady turbulent boundary layer near a free-slip surface. Phys. Fluids 21 (6).

Chaouat, B., 2001. Simulations of channel flows with effects of spanwise rotation or wall injection using a Reynolds stress model. J. Fluid Eng.-T. ASME 123, 2-10.

Choi, S.-K., Kim, S.-O., 2008a. Computation of a turbulent Rayleigh-Benard convection with the elliptic-blending secondmoment closure. Int. Commun. Heat Mass Transfer 35 (7), 817821.

Choi, S.-K., Kim, S.-O., 2008b. Treatment of turbulent heat fluxes with the elliptic-blending second-moment closure for turbulent natural convection flows. Int. J. Heat Mass Tran. 51 (9-10), 23772388.

Choi, S.-K., Kim, S.-O., 2012. Turbulence modeling of natural convection in enclosures: A review. J. Mech. Sci. Technol. 26 (1), 283-297. 
Table C.4: Modified equations, re-calibrated and additional coefficients (other coefficients are left unchanged), and figures in which the particular version is used.

\begin{tabular}{|c|c|c|}
\hline Modified equations & Modified coefficients & Figures \\
\hline$(\mathrm{C} .3) \rightarrow(\mathrm{C} .3)+g_{2} \varepsilon\left(b_{i k} b_{k j}-\frac{1}{3} b_{k l} b_{k l} \delta_{i j}\right)$ & $C_{L}=0.137, A_{1}=0.074, g_{2}=4.2$ & $1 \mathrm{a}, 8 \mathrm{~b}$ \\
\hline$(\mathrm{C} .7) \rightarrow \phi_{i j}^{w}=-\frac{\varepsilon}{k}\left(2 \overline{u_{i} u_{k}} n_{j} n_{k}+2 \overline{u_{j} u_{k}} n_{i} n_{k}+\frac{7}{2} \overline{u_{k} u_{l}} n_{k} n_{l} n_{i} n_{j}-\frac{5}{2} \overline{u_{k} u_{l}} n_{k} n_{l} \delta_{i j}\right)$ & $C_{L}=0.163, A_{1}=0.113$ & $1 \mathrm{a}, 2,3$ \\
\hline$(\mathrm{C} .2) \rightarrow \phi_{i j}^{*}=(1-k \alpha) \phi_{i j}^{w}+k \alpha \phi_{i j}^{h}$ & $C_{L}=0.4, A_{1}=0.175$ & $1 \mathrm{~b}$ \\
\hline$(\mathrm{C} .2) \rightarrow \phi_{i j}^{*}=\left(1-\alpha^{2}\right) \phi_{i j}^{w}+\alpha^{2} \phi_{i j}^{h}$ & $C_{L}=0.161, A_{1}=0.095$ & $1 \mathrm{~b}, 4 \mathrm{a}, 5 \mathrm{a}, \mathrm{b}$ \\
\hline$(\mathrm{C} .15) \rightarrow C_{\varepsilon 1}^{\prime}=C_{\varepsilon 1}\left[1+A_{1}\left(1-\alpha^{3}\right) \sqrt{\frac{k}{\overline{u_{i} u_{j}} n_{i} n_{j}}}\right]$ & $C_{L}=0.133, A_{1}=0.019$ & $1 c, 7 a, b$ \\
\hline$(\mathrm{C} .15) \rightarrow C_{\varepsilon 1}^{\prime}=C_{\varepsilon 1} ; \quad(\mathrm{C} .14) \rightarrow(\mathrm{C} .14)+C_{\varepsilon 3} \nu \frac{k}{\varepsilon} \overline{u_{j} u_{k}}\left(\frac{\partial^{2} U_{i}}{\partial x_{j} \partial x_{l}}\right)\left(\frac{\partial^{2} U_{i}}{\partial x_{k} \partial x_{l}}\right)$ & $C_{L}=0.133, C_{\varepsilon 3}=0.0665$ & $1 c, 7 a, b$ \\
\hline$(\mathrm{C} .15) \rightarrow C_{\varepsilon 1}^{\prime}=C_{\varepsilon 1} ; \quad($ C.14 $) \rightarrow($ C.14 $)+\left(1-\alpha^{3}\right) C_{\varepsilon 3} \nu \frac{k}{\varepsilon} \overline{u_{j} u_{k}}\left(\frac{\partial^{2} U_{i}}{\partial x_{j} \partial x_{l}}\right)\left(\frac{\partial^{2} U_{i}}{\partial x_{k} \partial x_{l}}\right)$ & $C_{L}=0.133, C_{\varepsilon 3}=0.0845$ & $1 \mathrm{c}, 7 \mathrm{a}, \mathrm{b}, 8 \mathrm{a}, \mathrm{b}$ \\
\hline$(\mathrm{C} .2) \rightarrow \phi_{i j}^{*}=(1-k \alpha) \phi_{i j}^{w}+k \alpha \phi_{i j}^{h} ;(\mathrm{C} .15) \rightarrow C_{\varepsilon 1}^{\prime}=C_{\varepsilon 1} ; \quad(\mathrm{C} .14) \rightarrow(\mathrm{C} .14)+C_{\varepsilon 3} \nu \frac{k}{\varepsilon} \overline{u_{j} u_{k}}\left(\frac{\partial^{2} U_{i}}{\partial x_{j} \partial x_{l}}\right)\left(\frac{\partial^{2} U_{i}}{\partial x_{k} \partial x_{l}}\right)$ & $C_{L}=0.45, C_{\varepsilon 3}=0.22$ & $4 \mathrm{a}, \mathrm{b}, 8 \mathrm{~b}$ \\
\hline$(\mathrm{C} .2) \rightarrow \phi_{i j}^{*}=\left(1-\alpha^{2}\right) \phi_{i j}^{w}+\alpha^{2} \phi_{i j}^{h} ; \quad(\mathrm{C} .15) \rightarrow C_{\varepsilon 1}^{\prime}=C_{\varepsilon 1}\left[1+A_{1}\left(1-\alpha^{3}\right) \sqrt{\frac{k}{\overline{u_{i} u_{j}} n_{i} n_{j}}}\right.$ & $C_{L}=0.161, A_{1}=0.03$ & $8 \mathrm{a}, \mathrm{b}$ \\
\hline None & $C_{L}=0.105$ & $8 \mathrm{a}$ \\
\hline $\operatorname{In}(\mathrm{C} .14), C_{\varepsilon 2} \rightarrow C_{\varepsilon 2}\left[1+\frac{A \sqrt{R e_{t}}}{C_{\varepsilon 2}\left(25+2 \omega^{*}\right)} \omega^{*}\right]$ & $A=0.1$ & $8 \mathrm{~b}$ \\
\hline
\end{tabular}

Choi, Y., Han, S., An, J., Shin, J., 2005. Predictions of turbulent heat transfer of super-critical carbon dioxide flow in a square duct with an elliptic-blending second moment closure. In: Proc. 4th Int. Symp. Turb. Shear Flow Phenomena, Williamsburg, VA, USA. Vol. 3. pp. 1101-1106.

Chou, P. Y., 1945. On velocity correlations and the solutions of the equations of turbulent fluctuation. Quart. of Appl. Math. 3, 38-54.

Dehoux, F., 2012. Modélisation statistique des écoulements turbulents en convection forcée, mixte et naturelle. Ph.D. thesis, university of Poitiers.

Dehoux, F., Benhamadouche, S., Manceau, R., 2011. Modelling turbulent heat fluxes using the elliptic blending approach for natural convection. In: Proc. 7th Int. Symp. Turb. Shear Flow Phenomena, Ottawa, Canada.

Dehoux, F., Lecocq, Y., Benhamadouche, S., Manceau, R., Brizzi, L.-E., 2012. Algebraic modeling of the turbulent heat fluxes using the elliptic blending approach. Application to forced and mixed convection regimes. Flow Turbul. Combust. 88 (1), 77-100.

Deville, M., Gatski, T., 2012. Mathematical Modeling for Complex Fluids and Flows. Springer.

Durbin, P. A., 1991. Near-Wall Turbulence Closure Modeling Without "Damping Functions". Theor. Comput. Fluid Dyn. 3, 1-13.

Durbin, P. A., 1993. A Reynolds stress model for near-wall turbulence. J. Fluid Mech. 249, 465-498.

Durbin, P. A., Laurence, D., 1996. Nonlocal Effects in Single Point Closure. In: Proc. 3rd Advances in Turbulence Research Conference. pp. 109-120.

Durbin, P. A., Speziale, C. G., 1991. Local Anisotropy in Strained Turbulence at High Reynolds Numbers. J. Fluid Eng.-T. ASME 113, 707-709.

Fadai-Ghotbi, A., Friess, C., Manceau, R., Borée, J., 2010a. A seamless hybrid RANS-LES model based on transport equations for the subgrid stresses and elliptic blending. Phys. Fluids 22 (055104).

Fadai-Ghotbi, A., Friess, C., Manceau, R., Gatski, T., Borée, J., 2010b. Temporal filtering: a consistent formalism for seamless hybrid RANS-LES modeling in inhomogeneous turbulence. Int. J. Heat Fluid Fl. 31 (3), 378-389.

Fadai-Ghotbi, A., Manceau, R., Borée, J., 2008. Revisiting URANS computations of the backward-facing step flow using second moment closures. Influence of the numerics. Flow Turbul. Combust. 81 (3), 395-414.

Fournier, Y., Bonelle, J., Moulinec, C., Shang, Z., Sunderland, A., Uribe, J., 2011. Optimizing Code_Saturne computations on Petascale systems. Comput. Fluids 45 (1), 103-108.
Grundestam, O., Wallin, S., Johansson, A., 2008. Direct numerical simulations of rotating turbulent channel flow. J. Fluid Mech. 598, 177-199.

Hallbäck, M., Johansson, A. V., 1993. Modelling of Rotation Effects in the $\varepsilon$-equation and Reynolds number Influence on slow pressure strain in RST closures. In: Proc. 5th Int. Symp. refined flow modelling and turbulence measurements, 1993. pp. 65-72.

Hanjalić, K., Launder, B., 2011. Modelling Turbulence in Engineering and the Environment. Second-Moment Routes to Closure. Cambridge University Press.

Ho, H., Don, C., Sik, S., Kun, S., 2007. Compressibility factor effect on the turbulence heat transfer of super-critical carbon dioxide by an elliptic-blending second moment closure. Trans. Korean Soc. Mech. Engineers, B 31 (1), 40-50.

Hunt, J. C. R., Graham, J. M. R., 1978. Free-stream turbulence near plane boundaries. J. Fluid Mech. 84 (2), 209-235.

Iaccarino, G., Ooi, A., Pettersson Reif, B. A., Durbin, P., 1999. RANS simulations of rotating flows. In: Ann. Res. Briefs. Center for Turbulence Research, Stanford University, CA, USA, pp. 257266.

Jacquin, L., Leuchter, O., Cambon, C., Mathieu, J., 1990. Homogeneous turbulence in the presence of rotation. J. Fluid Mech. 220, $1-52$.

Jakirlić, S., Hanjalić, K., Tropea, C., 2002. Modeling rotating and swirling turbulent flows: A perpetual challenge. AIAA J. 40 (10), 1984-1996.

Jakirlić, S., Maduta, R., 2014. On "steady" RANS modeling for improved prediction of wall-bounded separation. In: AIAA SciTech, 52nd Aerospace Science Meeting, Natonal Harbor, Maryland, USA. No. 0586.

Johnston, J. P., Halleen, R. M., Lezius, D. K., 1972. Effects of spanwise rotation on the structure of two-dimensional fully developed turbulent channel flow. J. Fluid Mech. 56 (3), 533-557.

Kim, J., 1989. On the structure of pressure fluctuations in simulated turbulent channel flow. J. Fluid Mech. 205, 421-451.

Kristoffersen, R., Andersson, H. I., 1993. Direct simulation of lowReynolds number flow in rotating channel. J. Fluid Mech. 256, 163-197.

Lamballais, E., 2014. Direct numerical simulation of a turbulent flow in a rotating channel with a sudden expansion. J. Fluid Mech. 745, $92-131$.

Lamballais, E., Lesieur, M., Métais, O., 1996. Effects of spanwise rotation on the vorticity stretching in transitional and turbulent channel flow. Int. J. Heat Fluid Fl. 17 (3), 324-332. 
Lamballais, E., Métais, O., Lesieur, M., 1998. Spectral-Dynamic Model for Large-Eddy Simulations of Turbulent Rotating Channel Flow. Theor. Comput. Fluid Dyn. 12, 149-177.

Launder, B. E., Reece, G. J., Rodi, W., 1975. Progress in the development of a Reynolds-stress turbulence closure. J. Fluid Mech. 68 (3), 537-566.

Launder, B. E., Tselepidakis, D. P., Younis, B. A., 1987. A secondmoment closure study of rotating channel flow. J. Fluid Mech. $183,63-75$.

Lecocq, Y., Manceau, R., Bournaud, S., Brizzi, L.-E., 2008. Modelling of the turbulent heat fluxes in natural, forced and mixed convection regimes. In: Proc. 7th ERCOFTAC Int. Symp. on Eng. Turb. Modelling and Measurements, Limassol, Cyprus.

Lozano-Durán, A., Jiménez, J., 2014. Effect of the computational domain on direct simulations of turbulent channels up to $R e_{\tau}=$ 4200. Phys. Fluids 26 (1).

Luca, I., Sadiki, A., 2008. New insight into the functional dependence rules in turbulence modelling. Int. J. Eng. Sci. 46 (11), 1053-1062.

Lumley, J. L., 1978. Computational Modeling of Turbulent Flows. In: Advances in Applied Mechanics. Vol. 18. Academic Press, NewYork, pp. 123-175.

Manceau, R., 2003. Accounting for wall-induced Reynolds stress anisotropy in Explicit Algebraic Stress Models. In: Proc. 3rd Symp. Turb. Shear Flow Phenomena, Sendai, Japan.

Manceau, R., 2005. An improved version of the Elliptic Blending Model. Application to non-rotating and rotating channel flows. In: Proc. 4th Int. Symp. Turb. Shear Flow Phenomena, Williamsburg, VA, USA.

Manceau, R., Carlson, J. R., Gatski, T. B., 2002. A rescaled elliptic relaxation approach: neutralizing the effect on the log layer. Phys. Fluids 14 (11), 3868-3879.

Manceau, R., Hanjalić, K., 2000. A new form of the elliptic relaxation equation to account for wall effects in RANS modelling. Phys. Fluids 12 (9), 2345-2351.

Manceau, R., Hanjalić, K., 2002. Elliptic Blending Model: A New Near-Wall Reynolds-Stress Turbulence Closure. Phys. Fluids 14 (2), 744-754.

Manceau, R., Perrin, R., Hadžiabdić, M., Benhamadouche, S., 2014. Investigation of the interaction of a turbulent impinging jet and a heated, rotating disk. Phys. Fluids 26 (3)

Manceau, R., Wang, M., Laurence, D., 2001. Inhomogeneity and anisotropy effects on the redistribution term in Reynolds-averaged Navier-Stokes modelling. J. Fluid Mech. 438, 307-338.

Mansour, N. N., Kim, J., Moin, P., 1988. Reynolds-stress and dissipation-rate budgets in a turbulent channel flow. J. Fluid Mech. 194, 15-44.

Moser, R. D., Kim, J., Mansour, N. N., 1999. Direct numerical simulation of turbulent channel flow up to $R e_{\tau}=590$. Phys. Fluids 11 (4), 943-945

Nagano, Y., Hattori, H., 2003. Direct numerical simulation and modelling of spanwise rotating channel flow with heat transfer. J. Turbul. 4, 1-15.

Nakabayashi, K., Kitoh, O., 2005. Turbulence characteristics of twodimensional channel flow with system rotation. J. Fluid Mech. $528,355-377$.

Naot, D., Shavit, A., Wolfshtein, M., 1973. Two-point correlation model and the redistribution of Reynolds stresses. Phys. Fluids $16,738-743$.

Nilsen, P., Andersson, H., 1990. Rotational effects on suddenexpansion flows. In: Proc. 1st ERCOFTAC Int. Symp. on Eng. Turb. Modelling and Measurements, Dubrovnic.

Oberlack, M., 2001. A unified approach for symmetries in plane parallel turbulent shear flows. J. Fluid Mech. 427, 299-328.

Oceni, A. G., Manceau, R., Gatski, T., 2010. Introduction of wall effects in explicit algebraic stress models through elliptic blending. In: Stanislas, M., Jimenez, J., Marusic, I. (Eds.), Progress in wall turbulence: Understanding and Modelling. Springer, pp. 287-297.

Pakhomov, M., Terekhov, V., 2013a. Numerical investigation of flow and heat transfer in a gas-droplet separated turbulent stream using the Reynolds Stress transfer model. Fluid Dyn. 48, 789-799.

Pakhomov, M., Terekhov, V., 2013b. Peculiarities of propagation of the dispersed phase in a gas-droplet flow downstream of a pipe sudden expansion. Tech. Phys. 58 (2), 185-191.

Pakhomov, M., Terekhov, V., 2013c. Second moment closure modelling of flow, turbulence and heat transfer in droplet-laden mist flow in a vertical pipe with sudden expansion. Int. J. Heat Mass Tran. 66, 210-222.

Perot, B., Moin, P., 1995. Shear-free turbulent boundary layers. Part 1. Physical insights into near-wall turbulence. J. Fluid Mech. 295, 199-227.

Pettersson, B. A., Andersson, H. I., 1997. Near-wall Reynolds-stress modelling in noninertial frames of reference. Fluid Dyn. Res. 19, 251-276.

Pope, S. B., 1975. A more general effective-viscosity hypothesis. J. Fluid Mech. 72, 331-340.

Rothe, P., Johnston, J., 1979. Free shear layer behavior in rotating systems. J. Fluid Eng.-T. ASME 101, 117-120.

Rubinstein, R., Zhou, Y., 1997. The Dissipation Rate Transport Equation and Subgrid-Scale Models in Rotating Turbulence. ICASE Report 97-63, NASA.

Sagaut, P., Cambon, C., 2008. Homogeneous Turbulence Dynamics. Cambridge University Press.

Sarkar, S., Speziale, C. G., 1990. A simple nonlinear model for the return to isotropy in turbulence. Phys. Fluids 2 (1), 84-93.

Shimomura, Y., 1993. Near-Wall Turbulent Flows. Elsevier Science, New York, Ch. Turbulence Modeling Suggested by System Rotation, pp. 115-123.

Shin, J. K., An, J. S., Choi, Y. D., Kim, Y. C., Kim, M. S., 2008. Elliptic relaxation second moment closure for the turbulent heat fluxes. J. Turbul. 9 (3), 1-29.

Shin, J.-K., Choi, Y.-D., An, J.-S., 2007. Numerical analysis of turbulent flow and heat transfer in a square sectioned U-bend duct by elliptic-blending second moment closure. J. Mech. Sci. Technol. 21 (2), 360-371.

Shin, J. K., Chun, K. H., Choi, Y. D., 2003. Refinement of a second moment closure by the elliptic blending equation and its application to turbulent rotating channel flows. J. Turbul. 4 (030), 1-22. Spencer, A. J. M., 1971. Theory of Invariants. In: Eringen, A. C. (Ed.), Continuum Physics. Vol. 1. Academic Press, New York.

Speziale, C., Mac Giolla Mhuiris, N., 1989. On the prediction of equilibrium states in homogeneous turbulence. J. Fluid Mech. 209, 591-615.

Speziale, C. G., 1989. Turbulence modeling in non-inertial frames of reference. Theor. Comput. Fluid Dyn. 1, 3-19.

Speziale, C. G., Gatski, T. B., 1997. Analysis and modelling of anisotropies in the dissipation rate of turbulence. J. Fluid Mech. 344, 155-180.

Speziale, C. G., Sarkar, S., Gatski, T. B., 1991. Modeling the pressure-strain correlation of turbulence: an invariant dynamical system approach. J. Fluid Mech. 227, 245-272.

Terekhov, V. I., Pakhomov, M. A., 2011. Particle dispersion and turbulence modification in a dilute mist non-isothermal turbulent flow downstream of a sudden pipe expansion. Journal of Physics: Conference Series 318.

Thielen, L., Hanjalić, K., Jonker, H., Manceau, R., 2005. Predictions of flow and heat transfer in multiple impinging jets with an ellipticblending second-moment closure. Int. J. Heat Mass Tran. 48 (8), 1583-1598

Thielen, L., Hanjalić, K., Manceau, R., Jonker, H., 2001. Turbulence modelling in a single normally impinging jet. In: Proc. ASMEPVP Conference, Atlanta, USA.

Törnblom, O., Johansson, A. V., 2007. A Reynolds stress closure description of separation control with vortex generators in a plane asymmetric diffuser. Phys. Fluids 19 (115108)

Vanpouille, D., 2013. Développement de modèles de turbulence pour les écoulements soumis à la flottabilité. Ph.D. thesis, University of Toulouse.

Visscher, J., Andersson, H., 2011. Particle image velocimetry measurements of massively separated turbulent flows with rotation. Phys. Fluids $23(7)$

Viswanathan, A., Tafti, D., 2007. Capturing effects of rotation in sudden expansion channels using detached eddy simulation. AIAA 
J. 45 (8), 2100-2102.

Viti, V., Huang, G., Bradshaw, P., 2007. Numerical study of stresstransport turbulence models: Implementation and validation issues. Comput. Fluids 36 (8), 1373-1383.

Waclawczyk, M., Pozorski, J., Minier, J.-P., 2004. Probability density function computation of turbulent flows with a new near-wall model. Phys. Fluids 16 (5), 1410-1422.

Weinstock, J., Burk, S., 1985. Theoretical pressure strain term: resistance to large anisotropies of stress and dissipation. J. Fluid Mech. 154, 429-443.

Wizman, V., Laurence, D., Kanniche, M., Durbin, P., Demuren, A., 1996. Modeling near-wall effects in second-moment closures by elliptic relaxation. Int. J. Heat Fluid Fl. 17 (3), 255-266.

Wu, X., Baltzer, J., Adrian, R., 2012. Direct numerical simulation of a $30 R$ long turbulent pipe flow at $R^{+}=685$ : Large-and very large-scale motions. J. Fluid Mech. 698, 235-281.

Yokojima, S., Shima, N., 2010. Applicability of elliptic-relaxation method to free-surface turbulence. Fluid Dyn. Res. 42 (3).

Yukawa, H., 1935. On the interaction of elementary particles. Proc.

Phys. Math. Soc. Japan 17 (48). 